Estimativas para entropia, extensões simbólicas e hiperbolicidade para difeomorfismos simpléticos e conservativos

\author{
Thiago Aparecido Catalan
}




\title{
Estimativas para entropia, extensões simbólicas e hiperbolicidade para difeomorfismos simpléticos e conservativos.
}

\author{
Thiago Aparecido Catalan
}

Orientador: Prof. Dr. Ali Tahzibi

Tese apresentada ao Instituto de Ciências Matemáticas e de Computação - ICMC-USP, como parte dos requisitos para obtenção do título de Doutor em Ciências - Matemática. VERSÃO REVISADA.

USP - São Carlos

Fevereiro de 2011 
Ficha catalográfica elaborada pela Biblioteca Prof. Achille Bassi e Seção Técnica de Informática, ICMC/USP,

\section{Catalan, Thiago}

Ccate Estimativas para entropia, extensões simbólicas e hiperbolicidade para difeomorfismos simpléticos e conservativos / Thiago Catalan; orientador Ali Tahzibi -- São Carlos, 2011.

$82 \mathrm{p}$.

Tese (Doutorado - Programa de Pós-Graduação em Matemática) -- Instituto de Ciências Matemáticas e de Computação, Universidade de São Paulo, 2011.

1. entropia. 2. extensão simbólica. 3. tangência homoclínica. 4. ciclos heterodimensionais. 5. conjectura de Palis. I. Tahzibi, Ali, orient. II. Título. 
Aos meus pais,

Walter e Angela, e meu irmão, Cristiano. 



\section{Agradecimentos}

O caminho para se conseguir algo na vida pode ser difícil, muito difícil, porém existem acontecimentos, momentos, e pessoas, com as quais fico muito feliz em dividir este momento, que fazem com que este caminho seja muito, mais muito melhor do que o próprio objetivo.

Primeiramente agradeço a minha família, pois por mais dito que seja "família é tudo", eu mais uma vez digo FAMÍlIA É TUDO. Pai, Mãe e Ticé valeu mesmo, vocês são a base, a garantia e o porquê.

No entanto, numa jornada você não tem a sua família do seu lado o tempo todo, e é aí que o mundo conspira. Felizmente, acho que ele conspirou ao meu favor! Começando pelas pessoas com quem tive a honra e o prazer de morar, TranQra-Rio Preto (Morera e Jaca) e Morada do Escorpião-São Carlos (Claudinei, Mateus, Kenji, Guilherme, Baguitão, Maranhão e Yuri). Galera, foi muito massa mesmo! Agora acordem porque a churrasqueira está acesa e a vizinha está brava, bora Saint Patrick?! Ah, mas alguém tem que ficar são!

Desculpem-me as outras gerações, mas foi uma honra participar da melhor época do ICMC-USP, pessoas que trabalham pesado, porém se divertem de verdade também. Foram muitas boas discussões matemáticas, e muitos bons momentos. Hartmann, Jean, Bahiano, Pink (trairão), Pimenta, John, Jordão, Cati, Caverna, Thaisinha, Micena, Charanga, Kolmogorov, valeu pessoal! Ah, alguém trouxe uma certidão aí? Estou precisando de três urgente!

A caminhada matemática também tem sido muito prazerosa, e isto se deve não só aos conhecimentos adquiridos mas também aos grandes amigos que fiz durante os vários eventos pelo mundo, os quais agradeço nas pessoas de Yuri Lima e Yuri Ki. E em particular, gostaria de agradecer ao Prof. Alexander Arbieto não só pelos conhecimentos passados, como também pela motivação e principalmente pela amizade.

Ao Prof. Ali Tahzibi, agradeço pela orientação, paciência, e principalmente pelos conselhos, não só matemáticos, que foram de suma importância nestes anos em São Carlos.

Aos funcionários do ICMC-USP, o meu muito obrigado.

Por fim, mas não menos importante, agradeço a CAPES e a FAPESP pelo fomento. 

Provamos que $C^{1}$-genericamente difeomorfismos simpléticos ou são Anosov ou possuem entropia topológica limitada por baixo pelo supremo sobre o menor expoente de Lyapunov positivo dos pontos periódicos hiperbólicos. Usando isto exibimos exemplos de difeomorfismos conservativos sobre superfícies que não são pontos de semicontinuidade superior para a entropia topológica. Provamos também que $C^{1}$-genericamente difeomorfismos simpléticos não Anosov não admitem extensões simbólicas.

Mudando de assunto, Hayashi estendeu um resultado de Mañé, provando que todo difeomorfismo $f$ que possui uma $C^{1}$-vizinhança $\mathcal{U}$, onde todos os pontos periódicos de qualquer $g \in \mathcal{U}$ são hiperbólicos, é de fato um difeomorfismo Axioma A. Aqui, provamos o resultado análogo a este no caso conservativo, e a partir deste é possível exibir uma demonstração de um fato "folclore", a conjectura de Palis no caso conservativo. 

We prove that a $C^{1}$-generic symplectic diffeomorphism is either Anosov or the topological entropy is bounded from below by the supremum over the smallest positive Lyapunov exponent of the periodic points. By means of that we give examples of area preserving diffeomorphisms which are not point of upper semicontinuity of entropy function in $C^{1}$-topology. We also prove that $C^{1}$-generic symplectic diffeomorphisms outside the Anosov ones do not admit symbolic extension.

Changing of subject, Hayashi has extended a result of Mañé, proving that every diffeomorphism $f$ which has a $C^{1}$-neighborhood $\mathcal{U}$, where all periodic points of any $g \in \mathcal{U}$ are hyperbolic, it is an Axiom A diffeomorphism. Here, we prove the analogous result in the volume preserving scenario, and using it we prove a "folklore" fact, the Palis conjecture in this context. 

Resumo

Abstract

1 Lemas de perturbação no mundo simplético e conservativo $\quad 7$

1.1 Lema de Franks . . . . . . . . . . . . . . . . . . . . . . . . . . 7

1.2 Lemas de conexão . . . . . . . . . . . . . . . . . . . . . . 10

1.3 Lemas de sombreamento . . . . . . . . . . . . . . . . . . . 11

2 Estimativas para entropia topológica e não existência de extensões simbólicas

2.1 Entropia e expoentes de Lyapunov dos pontos periódicos . . . . . . . . . . . 13

2.1 .1 Definindo o funcional s(.) . . . . . . . . . . . . . 13

2.1.2 Uma limitação superior para entropia de difeomorfismos genéricos dentro dos Anosovs . . . . . . . . . . . . . . . . . . . . . 14

2.1.3 Uma limitação superior genérica para entropia e a demonstração do Teorema A . . . . . . . . . . . . . . 16

2.2 Estimativa para a entropia de difeomorfismos simpléticos . . . . . . . . . . 17

2.2 .1 Demonstração do Teorema B . . . . . . . . . . . . . . 18

2.2.2 Demonstração do Teorema D . . . . . . . . . . . . . . . . . . 19

2.3 Perturbações simpléticas: demonstração da Proposição $2.2 .1 \quad \ldots . . \quad \ldots . \quad 23$

2.4 Exemplos de pontos de descontinuidade para entropia . . . . . . . . . . 31 
3 Hiperbolicidade no mundo conservativo 3

3.1 Índice das órbitas periódicas para difeomorfismos em $\mathcal{G}_{m}^{1}(M) \ldots \ldots 33$

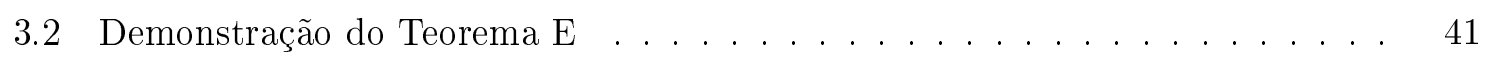

3.3 Conjectura de Palis no mundo conservativo . . . . . . . . . . . 47

$\begin{array}{ll}\text { A Proposição 2.1.3 } & 49\end{array}$

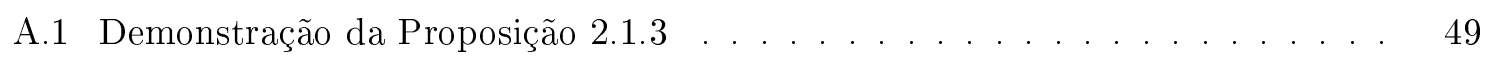

A.2 Demonstração da Proposição A.1.1 . . . . . . . . . . . . . . . 50

B Passo 1 da Proposição 2.2.1

$\begin{array}{ll}\text { Referências Bibliográficas } & 61\end{array}$

$\begin{array}{ll}\text { Tabela de Símbolos e Abreviações } & 65\end{array}$

$\begin{array}{ll}\text { Índice Remissivo } & 68\end{array}$ 


\section{Introdução}

A entropia topológica é um invariante muito importante em Sistemas Dinâmicos. Informalmente, a entropia topológica calcula o "número de trajetórias diferentes" do sistema. Formalmente, se $f$ é um homeomorfismo sobre uma variedade $M$ definimos-a da seguinte maneira

$$
h(f)=\lim _{\varepsilon \rightarrow 0} \limsup _{n \rightarrow \infty} \frac{1}{n} \log r(n, \varepsilon),
$$

onde $r(n, \varepsilon)$ é o máximo de órbitas $\varepsilon$-distintas com comprimento $n$. Duas órbitas com comprimento $n$ são $\varepsilon$-distintas se existe $0 \leq j \leq n$ tal que $d\left(f^{j}(x), f^{j}(y)\right)>\varepsilon$.

Para difeomorfismos Axiom A, Bowen [15] provou que a entropia determina o crescimento assintótico exponencial do número de pontos periódicos e por um resultado de Katok para qualquer difeomorfismo $C^{1+\alpha}(\alpha>0)$ sobre uma variedade de dimensão 2, a entropia é limitada superiormente por tal taxa de crescimento: $h(f) \leq \lim \sup _{n \rightarrow \infty} \frac{P_{n}(f)}{n}$.

Nesta tese, encontramos estimativas inferiores para entropia topológica de difeomorfismos simpléticos $C^{1}$-genéricos em termos dos expoentes de Lyapunov dos pontos periódicos do sistema (Veja Teoremas B, A).

Relacionamos tais estimativas para entropia com a (semi-continuidade) regularidade da função entropia com respeito a dinâmica do sistema (veja Teorema C) e construímos exemplos de difeomorfismos conservativos sobre superfícies que não são pontos de semi-continuidade da entropia topológica.

Também, usamos desta estimativa para provar a não existência de extensões simbólicas para difeomorfismos simpléticos $C^{1}$-genéricos longe dos difeomorfismos de Anosov. ( Veja Teorema D).

Mudando um pouco de enfoque, outra importante questão em Sistemas Dinâmicos é saber quando propriedades robustas no espaço de fase nos levam a propriedades diferenciáveis dos sistemas. Por hora, uma das mais importantes propriedades que o sistema pode ter é estabilidade. Isto é, qualquer sistema suficientemente próximo possui a mesma estrutura de órbitas do inicial. Em outras palavras, existe uma conjugação topológica entre este sistema e o inicial. 
Em um notável trabalho [36], Mañé provou que todo difeomorfismo $C^{1}$ estruturalmente estável é um difeomorfismo Axioma A. Em [41], Palis estendeu este resultado para difeomorfismos $\Omega$-estáveis. No entanto, Mañé acreditava que uma propriedade mais fraca do que $\Omega$-estabilidade seria suficiente para garantir a propriedade Axiom A, mais precisamente a existência apenas de pontos periódicos hiperbólicos robustamente. Nesta tese, provamos ser verdade esta "caracterização" no mundo conservativo (veja Teorema E) e usando esta caracterização para os difeomorfismos hiperbólicos provamos a conjectura de Palis no mundo conservativo (veja Teorema F).

Convém observarmos agora que apesar de não haver uma demonstração escrita em nenhum lugar até o presente momento a respeito desta conjectura no caso conservativo, a mesma já era assumida como sendo um fato "folclore"por alguns pesquisadores, veja [20].

Os resultados da tese estão divididos em dois capítulos, sendo o primeiro deles "Estimativas para entropia topológica e não existência de extensões simbólicas" em colaboração com o orientador do projeto Prof. Dr. Ali Tahzibi [19], e o segundo "Hiperbolicidade no mundo conservativo" em colaboração com o Prof. Dr. Alexander Arbieto da UFRJ [3].

\section{Estimativas inferiores para entropia topológica}

Primeiramente, Newhouse em [37] conseguiu uma limitação inferior para entropia topológica de difeomorfismos conservativos genéricos não Anosov sobre superfícies. Lembremos que um difeomorfismo $C^{1}$ sobre uma variedade Riemanniana $M$ é um difeomorfismo de Anosov se a variedade $M$ é um conjunto hiperbólico para $f$, onde um conjunto compacto $f$-invariante $\Lambda$ de $M$ é um conjunto hiperbólico se existe uma decomposição contínua $D f$-invariante $T_{\Lambda} M=$ $E^{s} \oplus E^{u}$ tal que existem constantes $0<\lambda<1$ e $C>0$, satisfazendo

$$
\left\|D f^{k} \mid E^{s}(x)\right\| \leq C \lambda^{k} \quad \text { e } \quad\left\|D f^{-k} \mid E^{u}(x)\right\| \leq C \lambda^{k},
$$

para todo $x \in \Lambda$ e $k>0$. Denotamos o conjunto dos difeomorfismos de Anosov por $\mathcal{A}$.

Seja $M$ uma superfície Riemanianna, compacta, conexa, munida de uma forma de volume $m$, e $\operatorname{Diff}_{m}^{1}(M)$ denotando o conjunto dos difeomorfismos $C^{1}$ conservativos, i.e., o conjunto formado pelos difeomorfismos que preservam a forma de volume $m$, munido da topologia $C^{1}$ uniforme. Continuando, sabendo que um ponto periódico $p$ de $f$ é hiperbólico se $D f_{p}^{\tau(p, f)}$ possui autovalores com valores absolutos diferente de um, onde $\tau(p, f)$ é o período do ponto periódico hiperbólico $p$, definimos

$$
s(f)=\sup \left\{\frac{1}{\tau(p, f)} \log \lambda(p, f)\right\}
$$

sobre todos os pontos periódicos hiperbólicos $p$ de $f$, onde $\lambda(p, f)$ é o valor absoluto do único autovalor de $D f_{p}^{\tau(p, f)}$ com valor absoluto maior do que um. Sendo assim, o resultado de Newhouse é o seguinte. 
Teorema 1. (Newhouse) Existe um subconjunto residual $\mathcal{B} \subset \operatorname{Diff}_{m}^{1}(M)$ tal que se $f \in \mathcal{B}$ é um difeomorfismo não Anosov, então

$$
h(f) \geq s(f) .
$$

Lembrando que um subconjunto residual é uma interseção enumerável de abertos e densos.

No entanto, conseguimos mostrar também que genericamente a desigualdade contrária também é verdadeira, o que implica o primeiro resultado desta tese.

Teorema A. Existe um subconjunto residual $\mathcal{B} \subset \operatorname{Diff}_{m}^{1}(M)$ (difeomorfismos conservativos sobre superfícies) tal que se $f \in \mathcal{B}$ é um difeomorfismo não Anosov, então

$$
h(f)=s(f) .
$$

Observemos que como corolário deste teorema e a semi-continuidade de $f \rightarrow s(f)$ (veja seção 2.1.1) concluímos que "genericamente" a entropia topológica é semi-contínua na topologia $C^{1}$. No entanto, não se sabe se os pontos de semi-continuidade para entropia topológica formam um subconjunto $C^{1}$-genérico.

É interessante mencionarmos que dentro dos difeomorfismos de Anosov para um subconjunto $C^{1}$-aberto e denso temos que $h(f)<s(f)$. Mais geral, a Proposição 2.1.1 nos dá uma limitação superior para entropia de difeomorfismos Anosov.

Em geral, podem existir difeomorfismos onde $h(f)>s(f)$. De fato, existem exemplos de difeomorfismos minimais com entropia positiva. Em dimensão dois existem homeomorfismos minimais com entropia positiva [43]. Entretanto, estes exemplos não preservam volume (e podem não formar um subconjunto $C^{1}$ - genérico).

Questão 1. Será que existem exemplos de difeomorfismos $C^{1}$ conservativos onde $h(f)>s(f)$ ?

Nosso próximo resultado é uma generalização do Teorema de Newhouse para simpléticos em geral. Seja $(M, \omega)$ uma variedade simplética Riemanianna, compacta, conexa, e denotemos por Diff ${ }_{\omega}^{1}(M)$ o espaço dos difeomorfismos simpléticos $C^{1}$ sobre $M$ munido da topologia $C^{1}$ uniforme. Lembrando que um difeomorfismo $f$ é simplético se ele preserva a 2-forma $\omega$, i.e., $f^{*} \omega=\omega$. Dado um ponto periódico hiperbólico $p$ de $f$, denotemos por $\lambda(p, f)$ o valor absoluto do menor autovalor de $D f_{p}^{\tau(p, f)}$ dentre os autovalores com valor absoluto maior do que um. Definindo

$$
s(f):=\sup \left\{\frac{1}{\tau(p, f)} \log \lambda(p, f)\right\}
$$

sobre todos os pontos periódicos hiperbólicos $p$ de $f$, nosso próximo resultado segue abaixo.

Teorema B. Existe um subconjunto residual no complementar dos difeomorfismos de Anosov $\mathcal{B} \subset \operatorname{Diff}_{\omega}^{1}(M)-\mathcal{A}$ tal que se $f \in \mathcal{B}$, então

$$
h(f) \geq s(f) .
$$




\section{Regularidade da Entropia}

Um importante problema em teoria ergódica suave é a regularidade da entropia com respeito a dinâmica. Por um resultado de Newhouse sabemos que $f \rightarrow h(f)$ é semi contínua superior na topologia $C^{\infty}$ para toda variedade sem bordo, e usando um resultado de Katok temos que a função entropia é de fato contínua na topologia $C^{\infty}$ para difeomorfismos sobre superfície. Nesta linha, temos o seguinte resultado.

Teorema C. Existem exemplos de difeomorfismos conservativos $C^{\infty}$ sobre superfície, $f_{0} \in$ $\operatorname{Diff}_{m}^{\infty}(M)$, tal que $f \rightarrow h(f)$ é sequer semi-contínua superior em $f_{0}$, no espaço dos difeomorfismos conservativos na topologia $C^{1}$.

\section{Extensões Simbólicas}

Dinâmica Simbólica exerce um papel crucial em teoria ergódica. É um problema desafiador saber se dinâmicas podem ser codificadas. Note que podemos obter limitações superiores para entropia por meio de dinâmica simbólica. Um sistema dinâmico $(M, f)$ possui uma extensão simbólica se existe um subshift $(Y, \sigma)$ e uma aplicação sobrejetiva $\pi: Y \rightarrow M$ tal que $\pi \circ \sigma=f \circ \pi$. $(Y, \sigma)$ é chamado uma extensão de $(M, f)$ e $(M, f)$ um fator de $(Y, \sigma)$. Note que, se um sistema possui uma extensão simbólica ganhamos uma limitação superior para entropia topológica do sistema, embora tais estimativas possam ser "exageradas". Neste sentido, uma extensão é chamada extensão simbólica principal se a aplicação $\pi$ é tal que $h_{\nu}(\sigma)=h_{\pi_{*} \nu}(f)$ para toda medida $\sigma$-invariante $\nu \in \mathcal{M}(\sigma)$ sobre $Y$, onde $h_{\nu}(\sigma)$ é a entropia métrica de $f$ com respeito a $\nu$.

Boyle, D. Fiebig, U. Fiebig [12] provaram que difeomorfismos assintoticamente $h$-expansivos possuem uma extensão simbólica principal. Onde difeomorfismos assintoticamente $h$-expansivos são aqueles com dinâmica local não muito complicada, mais precisamente, dado um difeomorfismo $f$, se $B_{\infty}(x, \varepsilon)=\left\{y \in M ; d\left(f^{j}(x), f^{j}(y)\right)<\varepsilon\right.$ para todo $\left.j \in \mathbb{N}\right\}$ então $f$ é assintoticamente $h$-expansivo se

$$
\lim _{\varepsilon \rightarrow 0} \sup _{x \in M} h\left(f \mid B_{\infty}(x, \varepsilon)\right)=0 .
$$

Agora, por um resultado de Buzzi [17] todo difeomorfismo $C^{\infty}$ sobre uma variedade compacta é assintoticamente $h$-expansivo e consequentemente possuem uma extensão principal. Também, recentemente D. Burguet [16] mostrou que todo difeomorfismo $C^{2}$ sobre superfícies possuem extensões simbólicas. Estes resultados dão uma resposta partial positiva para a conjectura de Downarowicz e Newhouse que acreditam que todo difeomorfismo $C^{r}(r \geq 2)$ admitem extensões simbólicas.

Lembremos agora que difeomorfismos hiperbólicos possuem extensões simbólicas desde que eles possuem partições de Markov. Mais geralmente, Diaz, Fisher, Pacífico e Vieitez [22] provaram que todo difeomorfismo parcialmente hiperbólico $C^{1}$ com dimensão central admitindo uma decomposição por subfibrados unidimensionais é assintoticamente $h$-expansivo 
e portanto possui uma extensão simbólica principal. Veja também [21]. Por outro lado, temos que os difeomorfismos com entropia topológica nula também são $h$-expansivos e logo possuem extensão principal. Sendo assim, tornam-se interessante estudos a respeito dos difeomorfismos $C^{1}$ que não possuem extensões simbólicas. Neste sentido, Downarowicz e Newhouse usando o Teorema 1 provaram em [23] que longe de difeomorfismos de Anosov, difeomorfismos $C^{1}$ genéricos que preservam área sobre superfícies não admitem extensões simbólicas. Nosso próximo resultado, é uma extensão deste para difeomorfismos simpléticos em geral.

Teorema D. Existe um subconjunto residual no complementar dos Anosov $\mathcal{B} \subset \operatorname{Diff}_{\omega}^{1}(M)-\mathcal{A}$ tal que se $f \in \mathcal{B}$, então $f$ não possui extensões simbólicas.

Usando este resultado podemos exibir uma demonstração fácil e curta da conjectura da estabilidade no mundo simplético.

Corolário 1. Um difeomorfismo $f \in \operatorname{Diff}_{\omega}^{1}(M)$ é estruturalmente estável se, e somente se, $f$ é Anosov.

Demonstração. Suponhamos que $f \in \operatorname{Diff}_{\omega}^{1}(M)$ seja estruturalmente estável. Por Zehnder [50], temos que os difeomorfismos $C^{\infty}$ são densos no mundo simplético, e como difeomorfismos $C^{\infty}$ possuem uma extensão simbólica principal, todos os difeomorfismos numa vizinhança de $f$ possuem extensões principais. Portanto, de acordo com o Teorema $\mathrm{D}$, isto só é possível se $f$ é Anosov.

Observação 1. A demonstração acima é baseada no Teorema D. No entanto, para provarmos este teorema usamos a abundância de tangências homoclínicas no complementar dos difeomorfismos de Anosov no mundo simplético, as quais já são obstruções para estabilidade. Porém, a priori a não existência de extensões simbólicas não possuem uma relação direta com tangências homoclinicas. Então, poderíamos concluir que "um mecanismo em topologia $C^{1}$ " que nos dê a não existência de extensões simbólicas implicaria a não estabilidade.

Por outro lado, recentemente fomos informados de que G.Liao, J.Yang e M. Viana [34] provaram que difeomorfismos $C^{1}$ longe de tangências homoclínicas possuem extensôes simbólicas.

\section{Hiperbolicidade no mundo conservativo}

Seja agora $M$ uma variedade Riemanianna, compacta, conexa, sem bordo, com dimensão $d$ e denotemos por $\operatorname{Diff}_{m}^{1}(M)$ o conjunto dos difeomorfismos que preservam a medida de Lebesgue $m$ induzida pela métrica Riemanniana. Como antes, olhamos este espaço com a topologia $C^{1}$.

No espaço dos difeomorfismos $C^{1}$ sobre $M$, $\operatorname{Diff}^{1}(M)$, podemos definir o conjunto $\mathcal{F}^{1}(M)$ como sendo o conjunto dos difeomorfismos $f \in \operatorname{Diff}^{1}(M)$ que possuem uma $C^{1}$-vizinhança $\mathcal{U} \subset \operatorname{Diff}^{1}(M)$ tal que se $g \in \mathcal{U}$ então todo ponto periódico de $g$ é hiperbólico. Em [27], 
Hayashi provou que todo difeomorfismo em $\mathcal{F}^{1}(M)$ é Axiom A. Lembremos que em dimensão dois, isto foi provado por Mañé [35], e no caso de fluxos sem singularidades por Gan e Wen em [25].

Observemos que no caso conservativo, a condição Axiom A é equivalente ao difeomorfismo ser Anosov, desde que todo ponto da variedade $M$ é um ponto não errante pelo Teorema de Recorrência de Poincaré. Sendo assim, uma questão natural é se os resultados de Hayashi e Mañé ainda são verdadeiros no cenário conservativo. Na verdade, parece que os argumentos de Mañé valem neste caso, especialmente as perturbações usadas. Mais ainda, usando resultados genéricos recentes no mundo conservativo, muitos dos argumentos da prova original podem ser evitados. O próximo resultado da tese é neste sentido. Observemos que Bessa e Rocha também possuem resultados análogos em [8] e junto com Ferreira em [7], num contexto de fluxos incompressíveis e Hamiltonianos, embora sejam em dimensões baixas (três e quatro, respectivamente).

Definimos o conjunto $\mathcal{G}_{m}^{1}(M)$ como sendo o conjunto dos difeomorfismos $f \in \operatorname{Diff}_{m}^{1}(M)$ que possuem uma $C^{1}$-vizinhança $\mathcal{U} \subset \operatorname{Diff}_{m}^{1}(M)$ tal que se $g \in \mathcal{U}$, então todo ponto periódico de $g$ é hiperbólico. Sendo assim, nosso principal resultado nesta direção é o seguinte.

Teorema E. Todo difeomorfismo em $\mathcal{G}_{m}^{1}(M)$ é Anosov.

Se a variedade é simplética, como feito anteriormente, podemos definir o conjunto $\mathcal{G}_{\omega}^{1}(M)$ usando apenas difeomorfismos simpléticos. Agora, desde que as vizinhanças dos difeomorfismos são tomadas respectivamente nos espaços $\operatorname{Diff}_{m}^{1}(M), \operatorname{Diff}_{\omega}^{1}(M)$, ou $\operatorname{Diff}^{1}(M)$ não possuímos uma relação entre $\mathcal{G}_{m}^{1}(M), \mathcal{G}_{\omega}^{1}(M)$ e $\mathcal{G}^{1}(M)$ direto da definição. No entanto, desde que Newhouse em [38] provou que todo elemento de $\mathcal{G}_{\omega}^{1}(M)$ é Anosov, usando o resultado de Hayashi e o Teorema E temos o seguinte.

Corolário 2. $\mathcal{G}_{\omega}^{1}(M) \subset \mathcal{G}_{m}^{1}(M) \subset \mathcal{F}^{1}(M)$.

Agora, como os argumentos para provar o Teorema E envolvem ciclos heterodimensionais, é natural tentarmos relacioná-lo com a conjectura de Palis [40]. Mais precisamente, o próximo resultado é uma prova desta conjectura no mundo conservativo.

Teorema F. Se $f \in \operatorname{Diff}_{m}^{1}(M)$ não é um difeomorfismo de Anosov então ele pode ser aproximado por um difeomorfismo ou exibindo um ciclo heterodimensional se a dimensão de $M$ é maior do que dois, ou exibindo uma tangência homoclínica se a dimensão de $M$ é dois.

O caso de superfícies é o resultado de Newhouse em [38], veja Apêndice B, desde que difeomorfismos conservativos sobre superfícies são simpléticos. 


西

\section{Lemas de perturbação no mundo simplético e conservativo}

\subsection{Lema de Franks}

Um dos principais lemas de perturbação na topologia $C^{1}$ é o Lema de Franks [24]. Este nos permite fazer perturbações não lineares ao longo de pedaços finitos de órbita, simplesmente usando argumentos de Álgebra Linear. Entretanto, no caso simplético e conservativo, são necessários argumentos mais específicos desde que a perturbação deve também preservar a estrutura inicial. No caso conservativo a ferramenta chave é o pasting Lema de ArbietoMatheus [4] e no caso simplético a teoria de funções geradoras faz este trabalho. Como antes, $M$ é uma variedade Riemanniana compacta, conexa e sem bordo.

Lema 1.1.1 (Lema de Franks). Seja $f \in \operatorname{Diff}_{\omega}^{1}(M)\left(\operatorname{Diff}_{m}^{1}(M)\right)$ e $\mathcal{U}$ uma $C^{1}$ vizinhança de $f$ em $\operatorname{Diff}_{\omega}^{1}(M)\left(\operatorname{Diff}_{m}^{1}(M)\right)$. Então, existe uma vizinhança $\mathcal{U}_{0} \subset \mathcal{U}$ de $f$ e $\delta>0$ tal que se $g \in \mathcal{U}_{0}(f), S=\left\{x_{1}, \ldots, x_{m}\right\} \subset M$ um pedaço finito de órbita qualquer e $\left\{L_{i}: T_{x_{i}} M \rightarrow\right.$ $\left.T_{x_{i+1}} M\right\}_{i=1}^{m}$ são aplicações lineares simpléticas (conservativas) satisfazendo $\left\|L_{i}-D g\left(x_{i}\right)\right\| \leq \delta$ para $i=1, \ldots m$, então existe $h \in \mathcal{U}(f)$ satisfazendo $h\left(x_{i}\right)=g\left(x_{i}\right)$ e $\operatorname{Dh}\left(x_{i}\right)=L_{i}$.

Observação 1.1.2. Como pode ser visto na demonstração do Lema 1.1.1, se U é uma vizinhança qualquer de $S$ então $h$ pode ser tomado tal que $h(x)=g(x)$ para todo $x \in S \cup(M-U)$.

Demonstração no caso simplético: 
Antes de mais nada, vamos definir as funções geradoras que são cruciais para realizarmos perturbações simpléticas locais. Seja $(u, v)=\left(u_{1}, \ldots, u_{n}, v_{1}, \ldots, v_{n}\right)$ um sistema de coordenadas em $\mathbb{R}^{2 n}$, e $\omega=\sum_{i=1}^{n} d u_{i} \wedge d v_{i}$ a 2-forma estandarte em $\mathbb{R}^{2 n}$. Seja $f(u, v)=$ $(\xi(u, v), \eta(u, v))$ um difeomorfismo simplético $C^{1}$ definido sobre uma vizinhança simplesmente conexa $V$ da origem. Então, $\sum_{i=1}^{n} d u_{i} \wedge d v_{i}=\sum_{i=1}^{n} d \xi_{i} \wedge d \eta_{i}$.

Suponhamos $f(0,0)=\left(\xi^{0}, \eta^{0}\right)$, e também assumamos que $\frac{\partial \eta(u, v)}{\partial v}$ seja não-singular em todo ponto de $V$. Desta forma, podemos olhar para $v$ como sendo uma função $C^{1}$ nas variáveis $u$ e $\eta$, i.e., $v=v(x, \eta)$.

Então, $\left(u_{1}, \ldots, u_{n}, \eta_{1}, \ldots, \eta_{n}\right)$ define novas coordenadas $C^{1}$ numa vizinhança pequena de $\left(0, \eta^{0}\right)$. Usando que $f$ é simplética, podemos ver que a 1 -forma $\alpha=\sum_{i=1}^{n} v_{i}(u, \eta) d u_{i}+$ $\xi(u, \eta) d \eta_{i}$ definida numa vizinhança de $\left(0, \eta^{0}\right)$ é fechada, i.e., $d \alpha=0$. Logo, existe uma função real $S=S(u, \eta)$, única a menos de uma constante, definida numa vizinhança de $\left(0, \eta^{0}\right)$ tal que $d S=\alpha$. $S$ é chamada de função geradora de $f$ e satisfaz o seguinte: $\frac{\partial S}{\partial \eta_{i}}=\xi_{i}, \frac{\partial S}{\partial u_{i}}=v_{i}$ e $\frac{\partial^{2} S}{\partial \eta_{i} \partial u_{i}}$ é não-singular para todo ponto $(u, \eta)$ próximo de $\left(0, \eta^{0}\right)$ no domínio de $S$.

Reciprocamente, se $S(u, \eta)$ é uma função real $C^{2}$ definida numa vizinhança de $\left(0, \eta^{0}\right)$ tal que $\frac{\partial^{2} S}{\partial \eta_{i} \partial u_{i}}$ é não-singular para todo ponto no domínio, então denotando $\xi_{i}(u, \eta)=\frac{\partial S}{\partial \eta_{i}}$ e $v_{i}(u, \eta)=\frac{\partial S}{\partial u_{i}}$ podemos encontrar $\eta=\eta(u, v)$ como uma função $C^{1}$ de $u$ e $v$, tal que $f(u, v)=(\xi(u, \eta(u, v)), \eta(u, v))$ seja um difeomorfismo simplético $C^{1}$ definido numa vizinhança da origem. O mais interessante e útil a respeito de funções geradoras é que difeomorfismos simpléticos são $C^{1}$ próximos se, e somente se, suas geradoras são $C^{2}$ próximas.

Provemos agora o lema de Franks. A ideia central na prova deste consiste em fazer boas perturbações da identidade.

Afirmação 1: Seja $L: \mathbb{R}^{2 n} \rightarrow \mathbb{R}^{2 n}$ uma transformação linear simplética $\delta_{0} C^{1}$-próxima da Id. Então, existe uma aplicação $R$ na bola $B(0, r)$ com centro na origem e raio $r$ arbitrariamente pequeno, $K_{0} \delta_{0}-C^{1}$ próximo da $I d$, tal que $R=I d$ numa vizinhança da fronteira da bola $B(0, r), R(0)=0$, e $D R(0)=L$.

Primeiro vamos construir tal perturbação para $r=1$. Sem perda de generalidade podemos escolher $\delta_{0}$ tal que $\frac{\partial L(u, v)}{\partial v}$ seja não-singular. Denotemos por $S_{L}(u, \eta)$ e $S_{I d}(u, \eta)$ as funções geradoras de $L$ e $I d$, respectivamente.

Seja $\beta$ uma função real bump $C^{\infty}$, que vale zero para $|t|>2 / 3$ e vale um para $|t|<1 / 2$. Denotando por $K_{1}=\sup \left\{\left|\beta^{\prime}\right|,\left|\beta^{\prime \prime}\right|\right\}$ e usando o fato das funções geradoras $S_{L}$ e $S_{I d}$ serem $\delta_{0}-C^{2}$ próximas, temos que

$$
S(x, \eta)=\beta(|(x, \eta)|) S_{L}(x, \eta)+(1-\beta(|(x, \eta)|)) S_{I d}(x, \eta)
$$

é $K_{1} K_{2} \delta_{0}-C^{2}$ próximo da identidade, onde $K_{2}$ é uma constante dependendo da dimensão de $M$. 
Então, o difeomorfismo simplético $\tilde{R}$ na bola $B(0,1)$ gerado por $S$ é $K_{1} K_{2} \delta_{0}-C^{1}$ próximo da $I d$. Mais ainda, $\tilde{R}=I d$ numa vizinhança da fronteira da bola $B(0,1), \tilde{R}(0)=0$ e $D \tilde{R}(0)=L$.

No caso geral, dado $r>0$ arbitrariamente pequeno, seja $\phi_{r}: B(0, r) \rightarrow B(0,1)$ uma homotetia, $\phi_{r}(p)=\frac{1}{r} p$. Sendo assim, $R=\phi_{r}^{-1} \circ \tilde{R} \circ \phi_{r}$ é um difeomorfismo simplético em $B(0, r), K_{1} K_{2} \delta_{0}-C^{1}$ próximo da $I d, R(0)=0$, e ainda $R=I d$ numa vizinhança da fronteira da bola $B(0, r)$. Tomando assim $K_{0}=K_{1} K_{2}$ temos a afirmação.

Observação 1.1.3. Usando o método de perturbação por funções geradoras como feito logo acima podemos, localmente, colar difeomorfismos simpléticos $C^{1}$ próximos através de perturbaçôes. Na verdade isto é nada mais, nada menos, que o pasting lema no caso simplético. Veja [4].

Continuando, seja $\varepsilon>0$ tal que todo $g 2 \varepsilon-C^{1}$ próximo de $f$ esteja em $\mathcal{U}$. Desta forma, escolhemos $\mathcal{U}_{0} \subset \mathcal{U}$ como sendo a $\varepsilon$-vizinhança de $f$ em $\operatorname{Diff}_{\omega}^{1}(M)$. Seja $\left(\psi_{i}, U_{i}\right)$ uma cobertura aberta de $M$ por coordenadas simpléticas. Lembre-se que coordenadas simpléticas , também conhecidas por coordenadas de Darboux, são tais que $\psi^{*} \omega$ é a 2-forma estandarte em $\mathbb{R}^{2 n}$.

Consideremos agora $r_{0}>0$ como sendo o número de Lebesgue para a cobertura tomada acima, e seja $0<r<r_{0}$ tal que as bolas de centro $x_{j}$ e raio $r, B\left(x_{j}, r\right)$, sejam duas a duas disjuntas. Sendo assim, usando as coordenadas simpléticas acima, podemos assumir tais bolas no espaço euclidiano.

Dado $g \in \mathcal{U}_{0}$, consideremos a seguinte aplicação linear $\tilde{L}_{j}=D g^{-1}\left(g\left(x_{j}\right)\right) L_{j}$ que em coordenadas simpléticas, por hipótese, é $C^{1}$ tão próxima da identidade quanto $L_{j}$ é próxima de $D g\left(x_{j}\right)$. Digamos que elas sejam $\varepsilon$ próximas.

Sendo assim, usando a Afirmação 1 para $\tilde{L}_{j}$, podemos encontrar $R_{j} K_{0} \varepsilon-C^{1}$ próximo da identidade tal que $R_{j}=I d$ numa vizinhança da fronteira da bola $B\left(x_{j}, r\right), R_{j}\left(x_{j}\right)=x_{j}$ e $D R_{j}\left(x_{j}\right)=\tilde{L}_{j}$. Sendo assim, consideremos $h_{j}$ o difeomorfismo simplético sobre $M$ tal que $h_{j}=R_{j}$ em $B\left(x_{j}, r\right)$ e igual a identidade no complementar desta. Agora, por escolha de $r$, temos que $\tilde{h}=h_{1} \circ \ldots \circ h_{m}$ é um difeomorfismo simplético em $M, K_{0} \varepsilon-C^{1}$ próximo da identidade. Mais ainda, $\tilde{h}=i d$ no complementar da união das bolas $B\left(x_{j}, r\right), \tilde{h}\left(x_{j}\right)=x_{j}$, e $D \tilde{h}\left(x_{j}\right)=\tilde{L}_{j}$. E assim, tomando $h=g \circ \tilde{h}$ e $\delta=\frac{\varepsilon}{K_{0}}$, temos o lema.

\section{Demonstração no caso conservativo}

A demonstração deste caso está contida em [32], e é basicamente uma aplicação do Pasting Lema de Arbieto-Matheus [4].

Teorema 1.1.4 (Pasting lema). Se $f$ é um difeomorfismo $C^{2}$ conservativo sobre $M$, e $x \in M$, então para todo $\varepsilon>0$ existe um difeomorfismo conservativo $g \varepsilon-C^{1}$ próximo de $f$ tal que para alguma vizinhança pequena $U \supset V$ de $x, g \mid U^{c}=f$ e $g \mid V=D f(x)$ (em coordenadas locais). 
Agora, como consequência deste resultado temos o seguinte lema.

Lema 1.1.5. Para todo $n \in \mathbb{N}$ e $\varepsilon>0$ existe uma vizinhança $\mathcal{G}$ da identidade em $S L(n, \mathbb{R})$, o subgrupo linear special, tal que para todo $A \in \mathcal{G}$ existe $h \in \operatorname{Diff}_{m}^{1}\left(\mathbb{R}^{n}\right)$ satisfazendo as seguintes propriedades:

1. $h$ coincide com a identidade no complementar da bola unitária na origem;

2. $h(0)=0$ e $D h(0)=A$;

3. $\|D h-I d\|<\varepsilon$.

Desta forma o a prova do lema de Franks no caso conservativo pode ser deduzida facilmente compondo $g$ com estes tipos de perturbações, como feito no caso simplético.

\subsection{Lemas de conexão}

Assim como o lema de Franks, outras perturbações locais são muito usadas no decorrer desta tese. Como exemplo destas temos as conexões de pontos próximos. O próximo resultado é neste sentido.

Lema 1.2.1. Dado um difeomorfismo $f \in \operatorname{Diff}_{\omega}^{1}(M)\left(\operatorname{Diff}_{m}^{1}(M)\right)$, existem constantes $\varepsilon_{0}>0 e$ $c>0$, dependendo apenas de $f$, tal que para todo $x \in M$, e qualquer $g \in \operatorname{Diff}_{\omega}^{1}(M)\left(\operatorname{Diff}_{m}^{1}(M)\right)$ $\varepsilon_{0}-C^{1}$ próximo de $f$, dados quaisquer números positivos $\delta, \varepsilon \in\left(0, \varepsilon_{0}\right)$ temos que se $d(y, x)<$ $c \delta \varepsilon$, então existe $h \in \operatorname{Diff}_{\omega}^{1}(M)\left(\operatorname{Diff}_{m}^{1}(M)\right) \varepsilon-C^{1}$ próximo de $f$ tal que $h\left(g^{-1}(x)\right)=y$ e $h=g$ no complementar de $g^{-1}\left(B_{\delta}(x)\right)$.

A demonstração deste lema no caso simplético é similar a do lema de Franks usando funções geradoras. Para o caso conservativo, usa-se o caso simplético em subespaços menores (lembre-se que funções simpléticas são conservativas) para se construir boas perturbações da identidade. Para maiores detalhes veja [47].

Nesta busca por perturbações de conexão, uma questão muito interessante colocada por Mañé é quando podemos conectar as variedades estável e instável por perturbações. É claro que neste caso as complicações são enormes desde que é preciso tomar-se um cuidado para garantir que os pontos ainda pertençam a estas variedades. Uma resposta positiva para tal foi dada por Hayashi [28]. No entanto, antes de enunciá-lo se $f$ é um difeomorfismo $C^{1}$ sobre $M$ e $p$ é um ponto periódico hiperbólico para $f$, definimos a variedade estável (instável) do ponto $p$ como sendo

$$
W^{s(u)}(p, f)=\left\{x \in M ; d\left(f^{n}(p), f^{n}(x)\right) \rightarrow 0 \text { quando } n \rightarrow+\infty(-\infty)\right\} .
$$

Teorema 1.2.2 $\left(C^{1}\right.$-Connecting Lema). Seja $f \in \operatorname{Diff}^{1}(M)$ e $p_{1}, p_{2}$ pontos periódicos hiperbólicos de $f$, tal que existam sequências de pontos $y_{n} \in M$ e números naturais $k_{n}$ tal que: 
- $\left.y_{n} \rightarrow y \in W_{l o c}^{u}\left(p_{1}, f\right)\right), y \neq p_{1} ; e$

- $\left.f^{k_{n}}\left(y_{n}\right) \rightarrow x \in W_{l o c}^{s}\left(p_{2}, f\right)\right), x \neq p_{2}$.

Então, existe um difeomorfismo $g$ arbitrariamente $C^{1}$-próximo de $f$ tal que $W^{u}\left(p_{1}, g\right)$ e $W^{s}\left(p_{2}, g\right)$ possuem uma interseção arbitrariamente próxima de $y$.

Apesar de termos enunciado o resultado na sua versão original (dissipativa) o mesmo foi provado ser verdade tanto no caso conservativo quanto simplético por Wen e Xia, veja [48].

\subsection{Lemas de sombreamento}

Como podemos ver na introdução desta tese, os pontos periódicos são de importância clara em sistemas dinâmicos. Neste sentido, temos o closing Lema de Pugh que nos permite aproximar pontos não errantes por pontos periódicos. Antes de enunciá-lo, lembremos que um ponto $x \in M$ é um ponto não errante para $f$ se para qualquer vizinhança $U$ de $x$, existe um inteiro positivo $k$ tal que $f^{k}(U) \cap U \neq \emptyset$, e denotemos por $\Omega(f)$ o conjunto dos pontos não errantes para $f$.

Teorema 1.3.1 (Closing Lema). Seja $f$ um difeomorfismo $C^{1}, z \in \Omega(f)$, e $\varepsilon>0$ arbitrariamente pequeno. Então, existe $k \in \mathbb{N}$ tal que para toda $C^{1}$ vizinhança $\mathcal{U}$ de $\underset{k}{f}$ existe um difeomorfismo $g \in \mathcal{U}$ tal que $z \in \operatorname{Per}(g)$ e ainda $f=g$ no complementar de $\bigcup_{-k}^{k} B_{\varepsilon}\left(f^{i}(z)\right)$, onde $B_{\varepsilon}(x)$ é a bola de centro $x$ e raio $\varepsilon$.

No entanto, tal resultado não garante uma "proximidade" entre a órbita do ponto não errante e a do ponto periódico. Para tal temos o Ergodic closing Lema de Mañé. Mais precisamente, seja $\Sigma(f)$ o conjunto dos pontos $x$ em $M$ tal que para toda vizinhança $\mathcal{U}$ de $f$ em $\operatorname{Diff}^{1}(M)$ e todo $\varepsilon>0$ existe um difeomorfismo $g \in \mathcal{U}$ e um ponto periódico $p \in \operatorname{Per}(g)$ tal que $d\left(f^{j}(x), g^{j}(p)\right)<\varepsilon, 0 \leq j \leq \tau(p, g)$, onde $\tau(p, g)$ é o período de $p$ para $g$.

Teorema 1.3.2 (Ergodic closing Lema). $\Sigma(f)$ é um conjunto de probabilidade total. Isto é, $\mu(\Sigma(f))=1$ para toda medida $\mu$ invariante por $f$.

Agora, tanto o closing Lema quanto o Ergodic closing Lema foram provados ser verdade no caso conservativo e simplético por M-C Arnaud, [5]. 



可

\section{Estimativas para entropia} topológica e não existência de extensões simbólicas

\subsection{Entropia e expoentes de Lyapunov dos pontos periódicos}

Nesta seção primeiro lembramos algumas definições e resultados, além de mostrarmos a existência de uma limitação superior para entropia topológica por expoentes de lyapunov para um subconjunto $C^{1}$-aberto e denso no conjunto dos difeomorfismos conservativos de Anosov. Depois, usando um resultado de Abdenur, Bonatti e Crovisier [1] provamos a existência de uma limitação superior para entropia num subconjunto $C^{1}$-genérico de $\operatorname{Diff}_{m}^{1}(M)$ (dimensão qualquer) e o usamos para provar o Teorema A.

\subsubsection{Definindo o funcional s(.)}

Dados $f \in \operatorname{Diff}_{\omega}^{1}(M)$ e um ponto periódico hiperbólico $p$ de $f$, denotamos por $\chi(p, f)$ o menor expoente de Lyapunov positivo do ponto periódico hiperbólico $p$, i.e., $\chi(p, f)=$ $1 / \tau(p, f) \log \lambda(p, f)$, onde $\lambda(p, f)=\left(\sigma\left(D f^{-\tau(p, f)} \mid E_{p}^{u}\right)\right)^{-1}$, sendo $\sigma$ o raio espectral da aplicação e $\tau(p, f)$ o período do ponto periódico hiperbólico $p$. Na verdade, definindo a medida 
periódica para $p$ por

$$
\mu_{p}=\frac{1}{\tau(p, f)} \sum_{i=0}^{\tau(p, f)-1} \delta_{f^{i}(p)},
$$

onde $\delta_{f^{i}(p)}$ é a medida de Dirac para $f^{i}(p)$, temos que $\chi(p, f)$ é o menor expoente de Lyapunov positivo para a medida ergódica $\mu_{p}$.

Agora, dado $n \in \mathbb{N}$ consideremos $s_{n}(f)=\max \left\{\chi(p, f) ; \quad p \in H_{n}(f)\right\}$, onde $H_{n}(f)$ é o conjunto dos pontos periódicos hiperbólicos de período menor ou igual a $n$. Como $H_{n}(f) \subset$ $H_{n+1}(f)$, temos que $s_{n}(f) \leq s_{n+1}(f)$, e então fica bem definido $s(f)=\lim _{n \rightarrow \infty} s_{n}(f)$. Da continuidade dos pontos periódicos hiperbólicos temos que o funcional $s_{n}$ é contínuo para todo $n \in \mathbb{N}$, o que implica que $s(f)$ é semi-contínua inferior.

\subsubsection{Uma limitação superior para entropia de difeomorfismos genéricos den- tro dos Anosovs}

O que mostramos nesta subseção é o seguinte.

Proposição 2.1.1. Existe um subconjunto $\mathcal{F} C^{1}$-aberto e denso dentro dos difeomorfismos de Anosov conservativos tal que para todo $f \in \mathcal{F}$ com $\operatorname{dim}\left(E^{u}\right)=u$ temos

$$
h(f)<\sup _{p \in \operatorname{Per}(f)} \sum_{i=1}^{u} \chi_{i}^{+}(p, f)
$$

onde a soma é sobre todos os expoentes de Lyapunov positivos de p para $f$.

Antes de demonstrarmos a proposição acima, convém observarmos que durante o texto quando dissermos que $f$ pode ser perturbada a fim de se obter alguma informação, quer dizer que em toda vizinhança de $f$ existe um difeomorfismo com esta propriedade. E normalmente, pensando em não complicar a notação, muitas vezes continuamos a denotar o difeomorfismo (perturbado) por $f$.

Demonstração. Seja $f$ um difeomorfismo de Anosov conservativo qualquer. Depois de uma $C^{1}$-perturbação, se necessário, podemos assumir que $f$ é um difeomorfismo de Anosov $C^{2}$. Isto é devido ao resultado de regularização de Avila [6].

Agora, sabemos que para difeomorfismos de Anosov conservativos a medida de Lebesgue $m$ é o único estado de equilíbrio para o potencial $\phi^{u}(x)=-\log J^{u} f(x), x \in M$, onde $J^{u} f(x):=|\operatorname{det} D f| E^{u}(x) \mid$. Para maiores detalhes veja [13]. Lembremos também que a medida que maximiza entropia é exatamente o estado de equilíbrio para o potencial identicamente nulo.

Sendo assim, usando o resultado de Bowen temos que $\mu$, a medida que maximiza entropia para $f$, coincide com a medida de Lebesgue se, e somente se, o potencial $\phi^{u}$ é cohmólogo a uma função constante. Então, perturbando $f$ na topologia $C^{1}$, podemos assumir que $\mu$ seja singular com respeito a medida de lebesgue (note que isto pode ser feito mantendo $f C^{2}$ ). 
Lembremos também que por Bowen, veja [14], a medida que maximiza entropia no caso Anosov é obtida como limite de distribuições periódicas. Isto é,

$$
\sum_{n}:=\frac{\sum_{p \in r_{n}(f)} \delta_{p}}{\# P e r_{n}(f)} \rightarrow \mu,
$$

onde $\operatorname{Per}_{n}(f)$ é o conjunto dos pontos periódicos de período $n$.

Sendo assim, por continuidade e definição de $\phi^{u}($.$) temos que$

$$
\int-\phi^{u}(x) d \mu(x)=\lim _{n \rightarrow \infty} \int-\phi^{u}(x) d \mu_{n}(x)
$$

e

$$
\int-\phi^{u} d \mu_{n} \leq \sup _{p \in P e r_{n}(f)} \sum_{i=1}^{u} \chi_{i}^{+}(p, f) .
$$

Logo,

$$
\int-\phi^{u} d \mu \leq \sup _{p \in \operatorname{Per}(f)} \sum_{i=1}^{u} \chi_{i}^{+}(p, f) .
$$

Como $f$ é um difeomorfismo Anosov $C^{2}$, a pressão de $\phi^{u}($.$) é zero. Sendo assim, usando$ o princípio variational temos

$$
\begin{aligned}
0=P_{f}\left(\phi^{u}\right) & =h_{m}(f)+\int \phi^{u} d m \\
& >h_{\mu}(f)+\int \phi^{u} d \mu \\
& =h(f)+\int \phi^{u} d \mu .
\end{aligned}
$$

E portanto, por (2.1)

$$
h(f)<\int-\phi^{u} d \mu \leq \sup _{p \in \operatorname{Per}(f)} \sum_{i=1}^{u} \chi_{i}^{+}(p, f) .
$$

Por fim, afirmamos que qualquer perturbação de $f$ também satisfaz uma desigualdade similar. De fato, como neste caso a entropia topológica é localmente constante (pela estabilidade estrutural dos difeomorfismos de Anosov) e $f \mapsto \sup _{p \in \operatorname{Per}(f)} \sum_{i=1}^{u} \chi_{i}^{+}(p, f)$ é uma função semicontínua inferior, concluímos que para todo $g C^{1}$-próximo de $f$ temos que

$$
h(g)<\sup _{p \in \operatorname{Per}(g)} \sum_{i=1}^{u} \chi_{i}^{+}(p, g) .
$$




\subsubsection{Uma limitação superior genérica para entropia e a demonstração do Teorema A}

Se $f$ é um difeomorfismo $C^{1}$ sobre $M$ e $\mu$ é uma medida ergódica para $f$, dizemos que $\mu$ é uma medida hiperbólica se os expoentes de Lyapunov de $\mu$ são todos não nulos.

Suponhamos agora $f$ um difeomorfismo $C^{1+\alpha}, \alpha>0$, e $\mu$ uma medida ergódica hiperbólica. Neste contexto, Katok provou que existe uma sequência de pontos periódicos $p_{n}$ tal que as medidas Dirac sobre as órbitas de $p_{n}$ convergem a $\mu$ e ainda os expoentes de Lyapunov de $p_{n}$ convergem aos expoentes de Lyapunov de $\mu$, [31]. Agora, lembremos que se $h_{\mu}(f)$ é a entropia métrica de $f$ com respeito a $\mu$, pelo princípio variational temos que $h(f)=\sup _{\mu} h_{\mu}(f)$, onde o supremo é tomado sobre as medidas ergódicas, e pela desigualdade de Ruelle temos que $h_{\mu}(f) \leq \sum \chi_{i}^{+}(\mu, f)$, onde a soma é sobre todos os expoentes de Lyapunov positivos de $\mu$. E assim, se o supremo no princípio variational fosse atingido por medidas hiperbólicas, então concluiríamos que

$$
h(f) \leq \sup _{p \in \operatorname{Per}(f)} \sum \chi_{i}^{+}(p, f) .
$$

O resultado seguinte nos diz que isto é, de fato, o que acontece no caso conservativo $C^{1}$-genérico.

Teorema 2.1.2. Existe um subconjunto residual $\mathcal{R} \subset \operatorname{Diff}_{m}^{1}(M)(M$ com dimensão qualquer d) tal que para todo $f \in \mathcal{R}$

$$
h(f) \leq \sup _{p \in \operatorname{Per}(f)} \sum_{i=1}^{n_{p}} \chi_{i}^{+}(p, f)
$$

onde a soma é sobre todos os expoentes de Lyapunov positivos do ponto periódico p para $f$, contando multiplicidade.

O Teorema A será uma consequência dos Teoremas 1 e 2.1.2. Vejamos assim, sua demonstração.

\section{Demonstração do Teorema A:}

Tomemos $\mathcal{R}$ como sendo o subconjunto residual resultado da interseção entre os subconjuntos residuais dados pelos Teoremas 1 e 2.1.2. Se o supremo no Teorema 2.1.2 fosse tomado sobre os pontos periódicos hiperbólicos então este sup em dimensão dois seria exatamente a função $s(f)$, e portanto o Teorema A estaria provado. A fim de superarmos isto, dividimos a prova em dois casos.

Dado um difeomorfismo $f \in \mathcal{R}$, se $h(f)=0$, então pelo Teorema 1 temos a igualdade buscada, $h(f)=s(f)$, desde que $s(f) \geq 0$. Por outro lado, como em dimensão dois, para um ponto periódico de um difeomorfismo conservativo ser hiperbólico é suficiente que ele possua um expoente de lyapunov positivo, se $h(f)>0$ temos que o supremo no Teorema 2.1.2 é de fato sobre os pontos periódicos hiperbólicos. Portanto temos a igualdade entre $h(f)$ e $s(f)$, o que concluí a demonstração. 
Para demonstrarmos o Teorema 2.1.2, vamos precisar do resultado seguinte de Abdenur, Bonatti e Crovisier em [1]. Antes de enunciá-lo, se $\mu$ é uma medida ergódica para um difemorfismo $f$ sobre $M^{d}$ definimos o vetor de Lyapunov $L(\mu)$ de $\mu$, como sendo o vetor $\left(\chi_{1}(\mu, f), \ldots, \chi_{d}(\mu, f)\right)$ em $\mathbb{R}^{d}$, sendo $\chi_{i}(\mu, f)$ os expoentes de Lyapunov de $\mu$ em ordem crescente.

Proposição 2.1.3. Existe um subconjunto residual $\mathcal{R} \subset \operatorname{Diff}_{m}^{1}(M)$ tal que se $f \in \mathcal{R}$ e $\mu$ é uma medida ergódica para $f$, então existem medidas periódicas $\mu_{p} \in \mathcal{M}(f)$ convergindo para $\mu$ na topologia fraca* ${ }^{*}$ e mais ainda os vetores formados pelos expoentes de Lyapunov de $\mu_{p}$, $L\left(\mu_{p}\right) \in \mathbb{R}^{d}$, também convergem ao vetor de Lyapunov $L(\mu) \in \mathbb{R}^{d}$.

$\mathrm{Na}$ verdade, eles provam este resultado no caso dissipativo, Teorema 3.8 em [1], no entanto os argumentos perturbativos usados lá também são válidos no caso conservativo. Veja Apêndice A para maiores detalhes.

Demonstração do Teorema 2.1.2: Seja $f \in \mathcal{R}$, onde $\mathcal{R}$ é o subconjunto residual dado na Proposição 2.1.3. Para qualquer $\varepsilon>0$, pelo princípio variational existe uma medida ergódica $\mu \in \mathcal{M}(f)$ tal que

$$
h(f)<h_{\mu}(f)+\varepsilon .
$$

Pela desigualdade de Ruelle $h_{\mu}(f) \leq \sum \chi_{i}^{+}(\mu, f)$, onde a soma é sobre todos os expoentes de Lyapunov positivos de $\mu$. Agora, usando a Proposição 2.1.3 podemos encontrar um ponto periódico $p$ de $f$ tal que $\sum \chi_{i}^{+}(\mu, f)<\sum \chi_{i}^{+}(p, f)+\varepsilon$. E então, temos que

$$
h(f)<\sup _{p \in \operatorname{Per}(f)} \sum \chi_{i}^{+}(p, f)+2 \varepsilon .
$$

Portanto, como $\varepsilon$ é arbitrariamente pequeno provamos o teorema.

\subsection{Estimativa para a entropia de difeomorfismos simpléticos}

Se $f$ é um difeomorfismo $C^{1}$ sobre uma variedade $M$, e $p$ é um ponto periódico hiperbólico de $f$, denotamos por $H(p, f)$ o conjunto dos pontos homoclínicos transversais de $p$, onde $q \notin o(p)$ é um ponto homoclínico transversal se ele é um ponto de interseção transversal entre $W^{s}(o(p), f)$ e $W^{u}(o(p), f)$. Se temos uma interseção não transversal entre estas variedades dizemos que $q$ é um ponto de tangência homoclínica. Lembremos que

$$
W^{s(u)}(o(p), f)=\bigcup_{i=0}^{\tau(p, f)-1} W^{s(u)}\left(f^{i}(p), f\right) .
$$

A partir de agora neste capítulo consideremos $(M, \omega)$ uma variedade Riemanianna simplética, compacta e conexa. Z. Xia em [47] provou que existe um subconjunto residual $\mathcal{H} \subset \operatorname{Diff}_{\omega}^{1}(M)$ tal que se $f \in \mathcal{H}$ e $p$ é um ponto periódico hiperbólico de $f$, então os pontos 
18Capítulo 2 - Estimativas para entropia topológica e não existência de extensões simbólicas

homoclínicos transversais de $p$ são densos nas variedades estável e instável da órbita de $p$, i.e., $W^{s}(o(p), f) \cup W^{u}(o(p), f) \subset \overline{H(p, f)}$. Vamos agora enunciar a proposição central deste capítulo e a partir dela provar os Teoremas B e D. A demonstração da proposição se encontra na próxima seção.

Lembremos que $\chi(p, f)=1 / \tau(p, f) \log \lambda(p, f)$, é o menor expoente de Lyapunov positivo do ponto periódico hiperbólico $p$ de $f$.

Proposição 2.2.1. (Proposição técnica principal) Seja p um ponto periódico hiperbólico de algum difeomorfismo não Anosov $f \in \mathcal{H}$. Dado $n>0$ e uma vizinhança qualquer $\mathcal{N} \subset$ $\operatorname{Diff}_{\omega}^{1}(M)$ de $f$, existe um subconjunto aberto $\mathcal{U} \subset \mathcal{N}$ tal que se $g \in \mathcal{U}$, então g possui um conjunto hiperbólico básico $\Lambda(p(g), n) \subset \overline{H(p(g), g)}$, onde $p(g)$ é a continuação do ponto periódico hiperbólico $p$ de $f$ para $g$, tal que as propriedades a seguir são verdadeiras

a) $h(g \mid \Lambda(p(g), n))>\chi(p(g), g)-\frac{1}{n}$.

b) Existe uma medida ergódica $\mu \in \mathcal{M}(\Lambda(p(g), n))$ tal que

$$
h_{\mu}(g)>\chi(p(g), g)-\frac{1}{n} .
$$

c) Para toda medida ergódica $\mu \in \mathcal{M}(\Lambda(p(g), n))$, temos que

$$
\rho\left(\mu, \mu_{p(g)}\right)<\frac{1}{n}
$$

onde $\rho$ é a métrica padrão que gera a topologia fraca*.

d) Para todo ponto periódico $q \in \Lambda(p(g), n)$, temos que

$$
\chi(q, g)>\chi(p(g), g)-\frac{1}{n} .
$$

Observação 2.2.2. Convém observarmos agora que para conseguirmos as perturbações acima passamos pela criação de intervalos de tangência homoclínica, o que a priori conseguimos fazer apenas na topologia $C^{1}$ (veja o Passo 2 da demonstração da Proposição 2.2.1). No entanto, no caso bidimensional através de resultados de Kaloshin [30] e Gonchenko-ShilnikovTuraev [26] é possivel conseguir intervalos de tangência, a partir de tangências homoclínicas, para perturbaçôes também em topologias altas, e usando isto Downarowicz e Newhouse [23] conseguiram também obter resultados de estimativas para entropia dentre outros para difeomorfismos genéricos sobre superfícies munidos da topologia $C^{r}, r \geq 2$.

\subsubsection{Demonstração do Teorema B}

Denotemos por $\mathcal{A}$ o conjunto dos difeomorfismos de Anosov e definimos $\mathcal{D}=\operatorname{Diff}_{\omega}^{1}(M)-\mathcal{A}$ como sendo o complementar do fecho dos difeomorfismos de Anosov no mundo simplético. 
Para inteiros positivos $n$ e $m$, seja $B_{n, m}$ o conjunto dos difeomorfismos $f$ em $\mathcal{D}$ tal que existam $p \in H_{n}(f)$ e um conjunto hiperbólico básico $\Lambda \subset \overline{H(p, f)}$, satisfazendo

$$
h(f \mid \Lambda)>s_{n}(f)-\frac{1}{m} .
$$

O Teorema B segue imediatamente da afirmação abaixo.

Afirmação: $B_{n, m}$ é um subconjunto aberto e denso de $\mathcal{D}$, para quaisquer inteiros positivos $n$ e $m$.

Para provarmos a afirmação, é suficiente encontrar abertos de $B_{m, n}$ próximo dos difeomorfismos $f \in \mathcal{D} \cap \mathcal{H}$, desde que estamos num espaço de Baire, e por Newhouse [38] todo difeomorfismo não Anosov pode ser aproximado por um difeomorfismo possuindo um ponto periódico 1-elíptico o qual é robusto no mundo simplético. Sendo assim, fixemos um difeomorfismo $f$ qualquer nesta interseção, e sejam $n, m \in \mathbb{N}$ quaisquer.

Por definição de $s_{n}$, existe $p_{0} \in H_{n}(f)$ tal que

$$
s_{n}(f)=\chi\left(p_{0}, f\right)
$$

Usando agora a Proposição 2.2.1, podemos encontrar $f_{1} C^{1}$-próxima de $f$ tal que $f_{1}$ possua um conjunto hiperbólico básico $\Lambda \subset \overline{H\left(p_{0}\left(f_{1}\right), f_{1}\right)}$, tal que

$$
h\left(f_{1} \mid \Lambda\right)>\chi\left(p_{0}\left(f_{1}\right), f_{1}\right)-\frac{1}{3 m} .
$$

Finalmente, usando a robustez de $\Lambda$ e $p_{0}$, a invariância da entropia topológica e que $s_{n}$ é contínua, temos que vale a seguinte desigualdade para difeomorfismos $g C^{1}$-próximos de $f_{1}$ :

$$
\begin{aligned}
h(g) & \geq h(g \mid \Lambda(g)) \\
& =h\left(f_{1} \mid \Lambda\right) \\
& >\chi\left(p_{0}\left(f_{1}\right), f_{1}\right)-\frac{1}{3 m} \\
& \geq \chi\left(p_{0}, f\right)-\frac{2}{3 m} \\
& =s_{n}(f)-\frac{2}{3 m} \\
& >s_{n}(g)-\frac{1}{m},
\end{aligned}
$$

o que prova a afirmação e portanto o Teorema B.

\subsubsection{Demonstração do Teorema D}

Lembremos que $(Y, \sigma)$ é uma extensão simbólica de $(M, f)$ se existe uma aplicação sobrejetiva $\pi: Y \rightarrow M$ tal que $\pi \circ \sigma=f \circ \pi$. Apesar desta semiconjugação nos dar uma limitação 
20Capítulo 2 - Estimativas para entropia topológica e não existência de extensões simbólicas

superior para entropia, esta pode passar informações exageradas com respeito a real dinâmica do sistema.

Em vista disto, definimos

$$
h_{\text {sex }}(f)= \begin{cases}\inf \{h(\sigma \mid Y):(Y, \sigma, \pi) \in S(f)\} & \text { se } S(f) \neq \emptyset \\ \infty & \text { se } S(f)=\emptyset\end{cases}
$$

onde $S(f)$ é o conjunto de todas as possíveis extensões simbólicas $(Y, \sigma, \pi)$ de $(M, f)$. Dizemos que $S(f)=\emptyset$ se não existem extensões simbólicas de $(M, f)$. Convém observar que as extensões simbólicas principais minimizam estas funções.

Definimos assim a entropia residual de um sistema da seguinte forma:

$$
h_{\text {res }}(f)=h_{\text {sex }}(f)-h(f) .
$$

A partir disto, precisamos mostrar que $h_{\text {sex }}(f)=\infty$ para todo difeomorfismo $f$ não Anosov num subconjunto residual $\mathcal{B} \subset \operatorname{Diff}_{\omega}^{1}(M)$ para provarmos o Teorema D.

Se $f: M \rightarrow M$ é um homeomorfismo sobre um espaço métrico compacto $M$, uma sequência crescente $\alpha_{1} \leq \alpha_{2} \leq \ldots$ de partições de $M$ é chamada essencial para $f$ se

1. $\operatorname{diam}\left(\alpha_{k}\right) \rightarrow 0$ quando $k \rightarrow \infty$, e

2. $\mu\left(\partial \alpha_{k}\right)=0$ para todo $\mu \in \mathcal{M}(f)$. Onde $\partial \alpha_{k}$ denota a união das fronteiras de todos os elementos da partição $\alpha_{k}$.

Uma sequência de partiçôes simpliciais é uma sequência encaixada $\mathcal{S}=\left\{\alpha_{1}, \alpha_{2}, \ldots\right\}$ de partições cujos diâmetros tendem a zero, e cada $\alpha_{k}$ é dada por uma triangularização suave de $M$. Pela Proposição 4.1 em [23] existe um subconjunto residual $\mathcal{R}_{\mathcal{S}} \subset \operatorname{Diff}_{\omega}^{1}(M)$ tal que se $f \in \mathcal{R}_{\mathcal{S}}$ então $\mathcal{S}$ é uma sequência essencial de partições de $M$ para $f$.

Sendo assim, para todo $k$ fixo, a função

$$
h_{k}(\mu)=h_{\mu}\left(\alpha_{k}\right),
$$

é o ínfimo de funções contínuas sobre $\mathcal{M}(f)$, e logo é semicontínua superior. Onde $h_{\mu}\left(\alpha_{k}\right)$ é a entropia da partição $\alpha_{k}$ por $f$. Usando estas funções e suas propriedades, Boyle e Downarowicz [11] provaram um resultado estrutural para extensões simbólicas, e a partir deste Downarowicz e Newhouse provaram em [23] o resultado seguinte que nos fornece um caminho para provar a não existência de extensões simbólicas.

Proposição 2.2.3 (Proposição 4.4 em [23]). Seja $f \in \mathcal{R}_{\mathcal{S}}$ e suponhamos que $\mathcal{E}$ seja um subconjunto compacto de $\mathcal{M}(f)$ tal que exista um número real positivo $\rho_{0}$, e para cada $\mu \in \mathcal{E}$ e $k>0$,

$$
\limsup _{\nu \in \mathcal{E}, \nu \rightarrow \mu}\left[h_{\nu}(f)-h_{k}(\nu)\right]>\rho_{0}
$$

Então,

$$
h_{\text {sex }}(f)=\infty .
$$


Lembremos que $H_{n}(f)$ denota o conjunto dos pontos periódicos hiperbólicos com período menor ou igual a $n$, e seja $H(f)=\cup_{n} H_{n}(f)$. Apesar de $H(f)$ poder ser um conjunto vazio, é sabido que o conjunto dos difeomorfismos $\mathcal{R}_{1}$ formado pelos difeomorfismos simpléticos $f$ tal que $H(f) \neq \emptyset$ é aberto e denso em $\operatorname{Diff}_{\omega}^{1}(M)$, veja [39]. Sendo assim, fica bem definido $\tau(f)$ como sendo o menor período dos pontos periódicos em $H(f)$ para todo $f \in \mathcal{R}_{1}$, e desta forma seja $\mathcal{R}_{1, m} \subset \mathcal{R}_{1}$ o conjunto dos difeomorfismos $f$ com $\tau(f)=m$. Notemos que $\mathcal{R}_{1}$ é uma reunião disjunta dos $\mathcal{R}_{1, m}$ 's.

Agora, para cada $f \in \mathcal{R}_{1}$ definimos

$$
\chi(f)=\sup \{\chi(p, f): p \in H(f) \text { e } \tau(p, f)=\tau(f)\} .
$$

Então, $\chi(f)$ depende continuamente de $f \in \mathcal{R}_{1}$ e $\chi(f)>0$.

Lembrando que $\mathcal{A} \subset \operatorname{Diff}_{\omega}^{1}(M)$ é o conjunto dos difeomorfismos de Anosov, seja $\mathcal{R}_{2, m}=$ $\mathcal{R}_{1, m} \backslash \mathcal{A}$. Logo $\mathcal{R}_{1} \backslash \mathcal{A}=\bigcup_{m} \mathcal{R}_{2, m}$.

Suponhamos agora que $\Lambda$ seja um conjunto periódico f-invariante com base $\Lambda_{1}$, i.e., $f^{k}\left(\Lambda_{1}\right)=\Lambda_{1}$ e $\Lambda=\cup_{0}^{k-1} f^{i}\left(\Lambda_{1}\right)$ para algum $k>0$, e seja $\alpha=A_{1}, A_{2}, \ldots, A_{s}$ alguma partição finita de $M$. Dizemos que $\Lambda$ é subordinado a $\alpha$ se para todo inteiro positivo $n$, existe um elemento $A_{i_{n}} \in \alpha$ tal que $f^{n}\left(\Lambda_{1}\right) \subset A_{i_{n}}$. Sendo assim, se $\mu \in \mathcal{M}(f \mid \Lambda)$ então $h_{\mu}(\alpha)=0$.

Dado um inteiro positivo $n$, dizemos que um difeomorfismo $f \in \operatorname{Diff}_{\omega}^{1}(M)$ satisfaz a propriedade $\mathcal{S}_{n}$ se para todo $p \in H_{n}(f) \operatorname{com} \chi(p, f)>\frac{\chi(f)}{2}$, o seguinte se verifica:

1. Existe um conjunto hiperbólico básico $\Lambda(p, n)$ para $f$ tal que

$$
\Lambda(p, n) \cap \partial \alpha_{n}=\emptyset \text { e } \Lambda(p, n) \text { é subordinado a } \alpha_{n} .
$$

3. Existe uma medida ergódica $\mu \in \mathcal{M}(\Lambda(p, n))$ tal que

$$
h_{\mu}(f)>\chi(p, f)-\frac{1}{n} .
$$

4. Para toda medida ergódica $\mu \in \mathcal{M}(\Lambda(p, n))$, temos que

$$
\rho\left(\mu, \mu_{p}\right)<\frac{1}{n} .
$$

5. Para todo ponto periódico $q \in \Lambda(p, n)$, temos

$$
\chi(q, f)>\chi(p, f)-\frac{1}{n} .
$$

E assim, para um inteiro positivo $m \leq n$ denotemos por $\mathcal{D}_{m, n}$ o subconjunto de $\mathcal{R}_{2, m}$ contendo os difeomorfismos $f$ satisfazendo a propriedade $\mathcal{S}_{n}$.

Desde que os pontos periódicos em $H_{n}(f)$ possuindo expoentes de lyapunov positivos maiores do que $\chi(f) / 2$ são finitos, temos direto da Proposição 2.2.1 que as condições (3), (4) e (5) acima são satisfeitas para difeomorfismos num subconjunto aberto e denso de $\mathcal{R}_{2, m}$. Agora, fixado uma partição $\alpha_{n}$, o subconjunto aberto $U \subset M$, onde é construído o conjunto 
22Capítulo 2 - Estimativas para entropia topológica e não existência de extensões simbólicas

hiperbólico $\Lambda(p, n)$ (veja demonstração da Proposição 2.2.1), pode ser tomado de tal forma que o conjunto $\Lambda(p, n)$ seja subordinado a $\alpha_{n}$. Portanto, como esta é uma propriedade robusta direto da Proposição 2.2.1 temos o seguinte lema.

Lema 2.2.4. Para inteiros positivos $m \leq n$, o subconjunto $\mathcal{D}_{m, n}$ é aberto e denso em $\mathcal{R}_{2, m}$.

Usando o lema acima vamos então demonstrar o Teorema D. Convém observar que usando o lema e a propriedade $S_{n}$ a demonstração a seguir é similar a demonstração do Teorema 1.3 em [23].

Demonstração do Teorema D: Seja

$$
\mathcal{R}_{2}=\bigcup_{m \geq 1} \bigcap_{n \geq m} D_{m, n}
$$

Pelo Lema 2.2.4, temos que $\mathcal{R}=\mathcal{R}_{\mathcal{S}} \cap \mathcal{R}_{2}$ é um subconjunto residual em $\mathcal{D}$.

O que vamos mostrar agora é que todo difeomorfismo $f \in \mathcal{R}$ não possui extensão simbólica, i.e., $h_{\text {sex }}(f)=\infty$.

Seja $f \in \mathcal{R}$ um difeomorfismo não Anosov. Definimos então

$$
\mathcal{E}_{1}=\left\{\mu_{p} ; \text { tal que } p \in H(f) \text { e } \chi(p, f)>\frac{\chi(f)}{2}\right\},
$$

e denotemos por $\mathcal{E}$ o seu fecho em $\mathcal{M}(f)$.

Usando este conjunto e as propriedades de $\mathcal{S}_{n}$ vamos mostrar que as hipóteses da Proposição 2.2.3 são satisfeitas para $\rho_{0}=\frac{\chi(f)}{2}$. Para tal, é suficiente verificarmos as hipóteses para todo $\mu_{p} \in \mathcal{E}_{1}$, e algum $k \in \mathbb{N}$ arbitrariamente fixado.

Sendo assim, dado $n \in \mathbb{N}$ grande o suficiente, como $f \in \mathcal{R}$ existe um conjunto básico hiperbólico $\Lambda(p, n)$ para $f$ subordinado a $\alpha_{n}$, e uma medida ergódica $\nu_{n} \in \mathcal{M}(\Lambda(p, n))$ tal que $\rho\left(\nu_{n}, \mu_{p}\right)<1 / n \mathrm{e}$

$$
h_{\nu_{n}}(f)>\chi(p, f)-\frac{1}{n} .
$$

Podemos supor sem perda de generalidade que $n>k$, e assim temos que $\alpha_{n}$ é mais fina do que $\alpha_{k}$ o que implica $\Lambda(p, n)$ ser também subordinado a $\alpha_{k}$. Agora, como $\nu_{n} \in \mathcal{M}(\Lambda(p, n))$

$$
h_{k}\left(\nu_{n}\right)=0 .
$$

Logo, usando isto juntamente com a desigualdade (2.3) temos que

$$
\left|h_{\nu_{n}}(f)-h_{k}\left(\nu_{n}\right)\right|=h_{\nu_{n}}(f)>\chi(p, f)-\frac{1}{n}>\rho_{0},
$$

onde a última desigualdade é satisfeita para valores grandes de $n$, desde que $\mu_{p} \in \mathcal{E}_{1}$.

E portanto, como $\nu_{n} \rightarrow \mu_{p}$, quando $n \rightarrow \infty$, para completarmos a demonstração falta mostrarmos que $\nu_{n}$ está de fato em $\mathcal{E}$, para todo $n$.

Como medidas ergódicas suportadas em conjuntos hiperbólicos são aproximadas por medidas periódicas, veja [45], temos que as medidas ergódicas $\nu_{n}$ são aproximadas por medidas 
periódicas. Mais precisamente, existem pontos periódicos hiperbólicos $q_{m, n} \in \Lambda(p, n)$ de $f$, tal que $\mu_{q_{m, n}}$ converge a $\nu_{n}$, quando $m \rightarrow \infty$, na topologia fraca*. Desta forma, nosso trabalho é reduzido a mostrar que $\mu_{q_{m, n}} \in \mathcal{E}_{1}$, o que é direto do fato de $f$ estar em $\mathcal{R}$ e o item 5 da propriedade $\mathcal{S}_{n}$. Provamos assim o Teorema D.

\subsection{Perturbações simpléticas: demonstração da Proposição 2.2.1}

Antes de demonstrarmos a proposição lembremos alguns fatos de estrutura simplética. Seja $(V, \omega)$ um espaço vetorial simplético de dimensão $2 n$. Dado um subespaço qualquer $W \subset V$ seu ortogonal simplético é definido como

$$
W^{\omega}=\{v \in V ; \omega(v, w)=0 \quad \text { para todo } \quad w \in W\} .
$$

O subespaço $W$ é chamado simplético se $W^{\omega} \cap W=\{0\}$. W e chamado isotrópico se $W \subset W^{\omega}$, isto é, $\omega \mid W \times W=0$. Um caso especial de subespaço isotrópico é um subespaço Lagrangiano, i.e., quando $W=W^{\omega}$.

Como antes $(M, \omega)$ é uma variedade simplética e seja $f$ um difeomorfismo simplético sobre $M$. A variedade estável (instável) de um ponto periódico hiperbólico de $f$ é uma subvariedade Lagrangiana de $M$, i.e., o espaço tangente a variedade estável (instável) no ponto $x$ é um subespaço Lagrangiano para todo ponto $x$ na variedade estável (instável) de um ponto periódico hiperbólico. Veja Afirmação 4 no Apêndice B.

Demonstração da Proposição 2.2.1: A demonstração está dividida em três passos onde o segundo e o terceiro são os principais e usam estruturas simpléticas.

Seja $f \in \mathcal{H}$ um difeomorfismo não Anosov.

Passo 1- Encontramos $g_{1} C^{1}$-próximo de $f$ tal que $p$ ainda seja um ponto periódico hiperbólico de $g_{1}$, e $g_{1}$ exiba uma tangência homoclínica entre $W^{s}\left(o(p), g_{1}\right)$ e $W^{u}\left(o(p), g_{1}\right)$. Mais ainda, $g_{1}=D f$ numa vizinhança pequena da órbita de $p$ (em coordenadas locais).

Passo 2- Encontramos $g_{2} C^{1}$-próximo de $g_{1}$ onde $g_{2}$ admite um intervalo de tangência homoclínica. Para tal, vão ser necessárias perturbações simpléticas em dimensões altas.

Passo 3- Finalmente, perturbamos $g_{2}$ para obtermos um difeomorfismo simplético $g$ satisfazendo as propriedades requeridas pela proposição. Mais ainda, $g$ é tal que as propriedades ainda são satisfeitas para difeomorfismos $C^{1}$-próximos dele, provando assim a proposição.

Demonstração do passo 1: Para criarmos a tangência homoclínica o caminho é exatamente o mesmo feito por Newhouse na demonstração da conjectura de Palis no caso simplético (passo 6, Teorema 1.1 em [38]). Feito isto, podemos usar o pasting lema e a continuidade de partes 
24Capítulo 2 - Estimativas para entropia topológica e não existência de extensões simbólicas

compactas das variedades estável e instável para obtermos uma tangência homoclínica e uma linearização numa vizinhança do ponto periódico hiperbólico. Observemos que devido a dimensão das variedades estável e instável, obter uma tangência homoclínica significa obter pelo menos uma direção comum (que pode ser única) entre os espaços tangente destas variedades no ponto de tangência. Pensando em manter o texto o mais completo possível, além de achar de grande valia a divulgação das técnicas envolvidas nesta demonstração vamos detalhá-la no Apêndice A.

Demonstração do passo 2: Para simplificarmos a notação suponhamos primeiro que $p$ seja um ponto fixo hiperbólico de $g_{1}$, e seja $V$ uma vizinhança de $p$ onde $g_{1}$, em coordenadas locais, é linear, com $E_{p}^{s}=\mathbb{R}^{n} \times\{0\}^{n}$ e $E_{p}^{u}=\{0\}^{n} \times \mathbb{R}^{n}$. Mais ainda, pelo Teorema de Darboux podemos também supor que em $V, \omega$ é a 2 -forma padrão para $\mathbb{R}^{2 n}, \omega=\sum d x_{i} \wedge d y_{i}$.

Seja $q$ o ponto de tangência homoclínica entre $W_{l o c}^{s}\left(p, g_{1}\right)$ e $W^{u}\left(p, g_{1}\right)$, tal que $q \in V$ e $g_{1}^{-1}(q) \notin V$. Sendo assim, podemos tomar uma vizinhança pequena $U \subset V$ de $q$ tal que $g_{1}^{-1}(U) \cap V=\varnothing$. Denotemos por $D$ a componente conexa de $W^{u}\left(p, g_{1}\right) \cap U$ que contém $q$.

Queremos agora perturbar $g_{1}$ a fim de conseguir um intervalo de tangência homoclínica. Como a variedade estável (instável) é gráfico, não é difícil fazer isto no caso conservativo usando o ponto de tangência $q$. No caso simplético isto pode ser feito usando o fato da variedade estável (instável) ser uma variedade lagrangiana como segue.

As perturbações feitas neste passo são feitas localmente em $U$, e portanto pensando em não complicar a notação vamos considerar uma outra coordenada simplética em $U$, tal que $q$ seja a origem e tenhamos o seguinte

$$
\begin{gathered}
W_{l o c}^{s}\left(p, g_{1}\right) \cap U=\left\{y_{1}=y_{2}=\ldots=y_{n}=0\right\} \cap U, \\
T_{q} D=\left\{y_{1}=x_{2}=\ldots=x_{n}=0\right\},
\end{gathered}
$$

e então

$$
W_{l o c}^{s}\left(p, g_{1}\right) \cap U \cap T_{q} D=\left\{e_{1}\right\},
$$

onde consideramos $\left\{e_{1}, \ldots, e_{n}, \ldots, e_{2 n}\right\}$ como sendo a base canônica de $\mathbb{R}^{2 n}$. Notemos que foi usado que $\operatorname{dim}\left(T_{q} W^{s}\left(p, g_{1}\right)+T_{q} W^{u}\left(p, g_{1}\right)\right)=2 n-1$, o que podemos supor ser verdade depois de uma perturbação, se necessário.

O lema seguinte é o ponto técnico e chave que nos permite construir um intervalo de tangência homoclínica para difeomorfismos simpléticos em dimensões altas.

Lema 2.3.1. Existe um difeomorfismo simplético $\phi: U \rightarrow \mathbb{R}^{2 n}$ sobre sua imagem, $C^{1}$ próximo da aplicação identidade Id numa vizinhança pequena de $q$, tal que $\phi(D) \cap W_{\text {loc }}^{s}\left(p, g_{1}\right) \cap U$ contém um segmento de reta.

Demonstração. Apenas aqui, usamos coordenadas $(x, y)$ com respeito a seguinte decomposição do espaço $\mathbb{R}^{2 n}=E \oplus F$, onde $E$ e $F$ são gerados por $\left\{e_{1}, e_{n+2}, \ldots, e_{2 n}\right\}$ e $\left\{e_{2}, \ldots, e_{n+1}\right\}$, respectivamente. Note que desta forma $E=T_{q} D$, e lembremos que $q=(0,0)$ por escolha da coordenada simplética em $U$. 
Como $D$ é localmente o gráfico de uma função com mesma classe de diferenciabilidade que $g_{1}$, existe uma aplicação $C^{1} j: B \subset \mathbb{R}^{n} \rightarrow \mathbb{R}^{2 n}, j(x)=(x, r(x))$, tal que $j(B)=D$. Mais ainda, $j$ é tal que $\operatorname{Dr}(0)=0$, e como $D \subset W^{u}\left(p, g_{1}\right)$ é uma variedade Lagrangiana, temos que $j^{*} \omega=0$, onde $j^{*} \omega$ é o pull-back da forma $\omega$ por $j$. Analogamente, se $i: \mathbb{R}^{n} \rightarrow \mathbb{R}^{2 n}$ é a inclusão natural, $i(x)=(x, 0)$, temos que $i^{*} \omega=0$ (lembremos que $\omega$ em $U$ é a 2-forma estandarte em $\left.\mathbb{R}^{2 n}\right)$.

Definimos agora $\phi: U \rightarrow \mathbb{R}^{2 n}$ por $\phi(x, y)=(x, y-r(x))$. Tomando $U$ menor, se necessário, é fácil ver que $\phi$ é de fato um difeomorfismo de $U$ sobre sua imagem e $C^{1}$ próximo da $I d$ numa vizinhança menor de $q=(0,0)$, desde que $\operatorname{Dr}(0)=0$. Sendo assim, para concluirmos o lema precisamos mostrar que $\phi$ é de fato simplético. Denotando a projeção da primeira coordenada por $\pi: \mathbb{R}^{2 n} \rightarrow \mathbb{R}^{n}, \pi(x, y)=x$, podemos reescrever $\phi$ da seguinte maneira $\phi=I d+i \circ \pi-j \circ \pi$. Então,

$$
\phi^{*} \omega=\omega+\pi^{*} i^{*} \omega-\pi^{*} j^{*} \omega=\omega,
$$

onde usamos que $i^{*} \omega=j^{*} \omega=0$ na segunda igualdade. Portanto, o lema está provado.

Voltando a demonstração do passo 2, podemos usar o pasting lema no caso simplético, Observação 1.1.3, e a aplicação $\phi$ dada pelo Lema 2.3.1, para encontrar $R: U \rightarrow U C^{1}$ próximo da $I d, \operatorname{com} R=\phi$ em alguma vizinhança de $q$, e $R=I d$ no complementar de outra vizinhança pequena contendo a anterior. Fica portanto bem definido $\tilde{R}: M \rightarrow M$, com $\tilde{R}=I d$ em $U^{c}$ e $\tilde{R}=R$ em $U$, e assim tomando $g_{2}=\tilde{R} \circ g_{1}$ temos uma $C^{1}$-perturbação de $g_{1}$ que coincide com $g_{1} \mathrm{em}\left(g_{1}^{-1}(U)\right)^{c}$. O diferencial desta perturbação é que ela exibe um intervalo de tangência homoclínica como queríamos. Mais precisamente, existe um segmento de reta $I \subset W_{\text {loc }}^{s}\left(p, g_{2}\right) \cap W^{u}\left(p, g_{2}\right) \cap U$. Para uso futuro, observemos que $I$ está contido no espaço gerado pelo vetor unitário $e_{1}$, e a menos de uma mudança de coordenadas em $U$, podemos supor $I \subset\left\{\left(x_{1}, 0, \ldots, 0\right),-2 a \leq x_{1} \leq 2 a\right\}$, para algum $a>0$ pequeno o suficiente $\mathrm{e}$ coordenadas usuais de $\mathbb{R}^{2 n}$.

Demonstração do passo 3: A idea agora é usar o intervalo de tangência para criarmos conjuntos hiperbólicos com as propriedades requeridas na proposição. Para tal, seja $N$ um número inteiro positivo grande e $\delta>0$ um número real arbitrariamente pequeno. Como antes (construção de $\tilde{R}$ ) podemos encontrar um difeomorfismo $\Theta: M \rightarrow M, \delta-C^{1}$ próximo da $I d$, $\Theta=I d$ em $U^{c} \mathrm{e}$

$$
\Theta(x, y)=\left(x_{1}, \ldots, x_{n}, y_{1}+A \cos \frac{\pi x_{1} N}{2 a}, y_{2}, \ldots, y_{n}\right), \text { para }(x, y) \in B(0, r) \subset U,
$$

com $A=\frac{2 K a \delta}{\pi N}$ e $r>0$ pequeno o suficiente, onde $K$ é uma constante dependendo apenas da coordenada simplética em $U$. Sendo assim, $g=\Theta \circ g_{2}$ é $\delta-C^{1}$ próximo de $g_{2}$, e mais, $g=g_{2}$ no complementar de $g_{2}^{-1}(U)$. Apesar do difeomorfismo $g$ depender de $N$, denotamos sempre estes difeomorfismos por $g$. É importante salientar que esta perturbação é uma adaptação da perturbação "Newhouse's snake" para dimensões maiores, i.e., ela destrói o intervalo de tangência homoclínica criando $N$ pontos homoclínicos transversais de $p$ em $U$. 
26Capítulo 2 - Estimativas para entropia topológica e não existência de extensões simbólicas

Usando a função $\Theta$ escolhemos agora estrategicamente dois pontos na variedade instável de $p$ para $g, z_{1}=\Theta(-a, 0, \cdots, 0)$ e $z_{2}=\Theta(a, 0, \cdots, 0)$. Agora, tomamos $\gamma_{1}$ e $\gamma_{2}$ dois discos transversais à variedade instável $W^{u}(p, g)$ nos pontos $z_{1}$ e $z_{2}$, respectivamente.

De agora em diante, voltamos a usar a coordenada simplética em $V$ fixada no começo da demonstração. Convém observar que $g$ é igual a $g_{1}$ em $V$, e portanto $g$ é linear em $V$.

Dado um conjunto $E$, denotemos por $C(E, x)$ a componente conexa de $E$ contendo $x$. Pelo $\lambda$-lema e a escolha de $\gamma_{1}$ e $\gamma_{2}, C\left(g^{-j}\left(\gamma_{1}\right) \cap V, g^{-j}\left(z_{1}\right)\right)$ e $C\left(g^{-j}\left(\gamma_{2}\right) \cap V, g^{-j}\left(z_{2}\right)\right)$ se acumulam sobre $W_{l o c}^{s}(p, g)$ para valores grandes de $j>0$.

Sendo assim, tomando $D^{s}=W_{l o c}^{s}(p, g) \cap U$ podemos definir o retângulo $D_{j}=D^{s} \times D_{j}^{u}$, para $j$ grande o suficiente, como sendo o produto cartesiano entre $D^{s}$ e $D_{j}^{u}$, onde $D_{j}^{u}$ é o menor disco possível em $\left\{\left(0, \ldots, 0, y_{1}, \ldots, y_{n}\right), y_{i} \in \mathbb{R}\right\}$ tal que $\pi_{2}\left(C\left(g^{-j}\left(\gamma_{i}\right) \cap V, g^{-j}\left(z_{i}\right)\right)\right) \subset D_{j}^{u}$, para $i=1,2$. Aqui, $\pi_{2}(x, y)=y$ é a projeção na segunda $n$-esima coordenada de $\mathbb{R}^{2 n}$. Lembremos que estamos considerando $V$ dentro do espaço euclidiano e ainda $E_{p}^{s}=\mathbb{R}^{n} \times\{0\}^{n}$ e $E_{p}^{u}=\{0\}^{n} \times \mathbb{R}^{n}$.

Seja $J \subset U$ algum disco pequeno na variedade instável $W^{u}(p, g)$ contendo os $N$ pontos homoclínicos transversais construídos anteriormente, e seja $T>>$ tal que $g^{-T}(J) \subset V$, e ainda $g^{-T}\left(\gamma_{i}\right), i=1,2$, esteja suficientemente próximo de $W_{l o c}^{s}(p, g)$. Denotemos por $\tilde{\Gamma}$ a $A / 2$-vizinhança de $J$, e definimos $\Gamma=g^{-T}(\tilde{\Gamma})$, veja figura 2.1 .

Agora, seja $t_{0}$ o menor inteiro positivo tal que $C\left(g^{-t_{0}}\left(\gamma_{i}\right), g^{-t_{0}}\left(z_{i}\right)\right)$ é $A / 2-C^{1}$ próximo de $W_{l o c}^{s}(p, g), i=1,2$. Notemos que se $t^{\prime} \geq t_{0}$, e $g^{t^{\prime}-T}\left(D_{t^{\prime}}\right) \subset \Gamma$, então $g^{t^{\prime}}\left(D_{t^{\prime}}\right) \cap\left(D_{t^{\prime}}\right)$ contém $N$ componentes conexas disjuntas, desde que $A$ é muito pequeno. Sendo assim, consideremos $z_{3}=(b, 0, \ldots, 0)$ e $z_{4}=\left(b^{\prime}, 0, \ldots, 0\right)$ dois pontos na variedade estável local de $p$, onde $b$ e $b^{\prime}$ são as extremidades esquerda e direita, respectivamente, de $W_{l o c}^{s}(p, g) \cap U$ olhando para a primeira coordenada. Como antes, seja $\gamma_{3}$ e $\gamma_{4}$ dois discos transversais a $W_{l o c}^{s}(p, g)$ nos pontos $z_{3}$ e $z_{4}$, respectivamente. Pelo $\lambda$-lema novamente podemos definir $t_{1}$ como sendo o menor inteiro positivo possível tal que

$$
C\left(g^{t_{1}}\left(\gamma_{i}\right), g^{t_{1}}\left(z_{i}\right)\right) \cap C\left(g^{-T}\left(\gamma_{j}\right), g^{-T}\left(z_{j}\right)\right) \cap \Gamma \neq \emptyset, \text { para } j=1,2 \text { e } i=3,4 .
$$

Finalmente, definimos $t=\max \left\{t_{0}, t_{1}+T\right\}$. Notemos que $t$ depende de $N$ desde que $t_{0} \mathrm{e}$ $t_{1}$ dependem, mais ainda $t$ vai para infinito quando $N$ vai.

Pelos comentários anteriores e escolha de $t$, temos que $g^{t}\left(D_{t}\right) \cap D_{t}$ possui $N$ componentes conexas disjuntas, e ainda $t$ é o menor possível tal que $D_{t}$ é $A / 2-C^{1}$ próximo de $W_{l o c}^{s}(p, g)$ e $g^{t}\left(D_{t}\right)$ é $A / 2-C^{1}$ próximo de $J \subset W^{u}(p, g)$. Portanto, desde que temos uma ferradura com $N$ pernas, o conjunto maximal invariante em $D_{t}$ para $g^{t}$

$$
\tilde{\Lambda}(p, N)=\bigcap_{j \in \mathbb{Z}} g^{t j}\left(D_{t}\right)
$$

é um conjunto hiperbólico com dinâmica conjugada ao shift de $N$ símbolos.

Logo, $h\left(g^{t} \mid \tilde{\Lambda}(p, N)\right)=\log N$ e tomando

$$
\Lambda(p, N)=\bigcup_{j=0}^{t-1} g^{j}(\tilde{\Lambda}(p, N))
$$




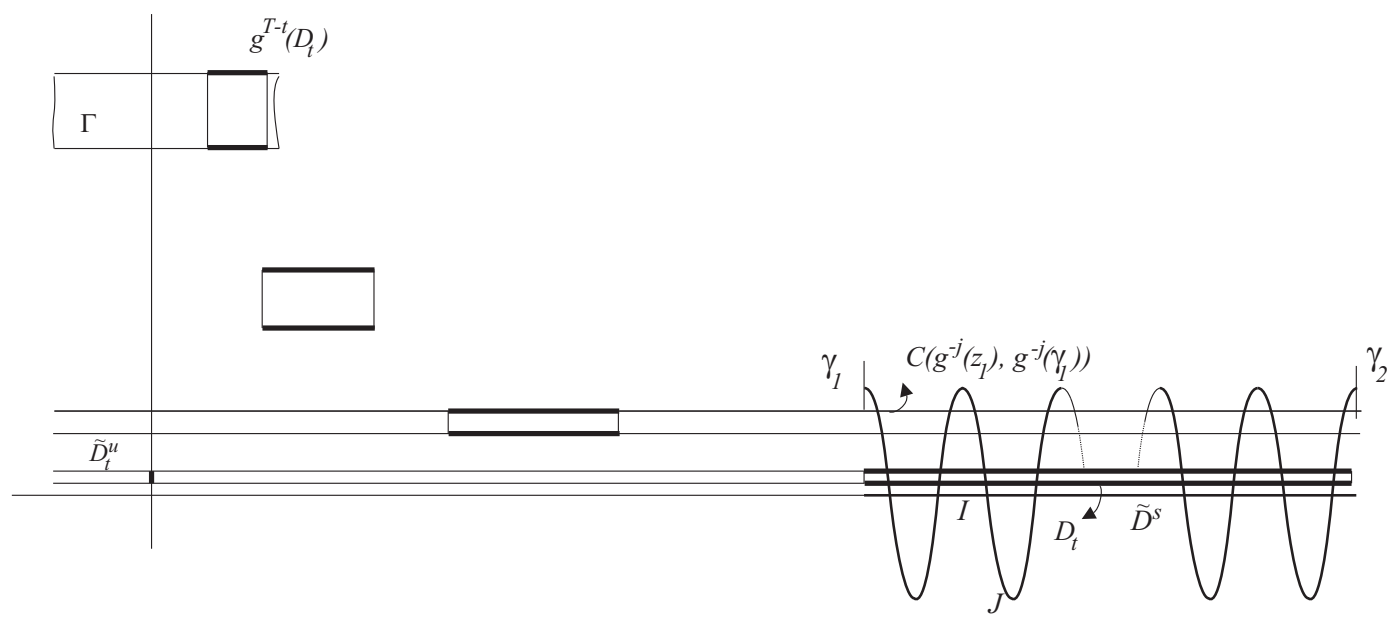

Figura 2.1:

temos que $h(g \mid \Lambda(p, N))=\frac{1}{t} \log N$.

O lema seguinte é o ponto chave neste passo.

Lema 2.3.2. Para $A$ e $t$ definidos como acima, existe um inteiro positivo $K_{1}$ independente de A, tal que

$$
A<K_{1} \min \left\{\left\|D g_{p}^{-t}\left|E^{u}\|,\| D g_{p}^{t}\right| E^{s}\right\|\right\}
$$

Demonstração. Primeiro, lembremos que $V$ é uma vizinhança de $p$ onde $g$ é linear. Portanto, se $m$ é o maior possível tal que $g^{j}(x) \in V$ para $0 \leq j \leq m$, então existem constantes $K_{2}$ e $K_{3}$ dependendo da coordenada simplética em $V$ tal que

$$
K_{2}\left\|D g_{p}^{m}\left|E^{u}\left\|^{-1} \leq d\left(x, W_{l o c}^{s}(p, g)\right) \leq K_{3}\right\| D g_{p}^{-m}\right| E^{u}\right\| .
$$

Analogamente, se $m$ é o maior possível tal que $g^{-j}(x) \in V$ para $0 \leq j \leq m$, então existem constantes $K_{4}$ e $K_{5}$ tal que

$$
K_{4}\left\|D g_{p}^{-m}\left|E^{s}\left\|^{-1} \leq d\left(x, W_{l o c}^{u}(p, g)\right) \leq K_{5}\right\| D g_{p}^{m}\right| E^{s}\right\| .
$$

Agora, pela escolha de $t$, ou existe $z \in D_{t}$ tal que

$$
d\left(g(z), W_{l o c}^{s}(p, g)\right) \geq A / 2
$$

ou existe $z \in g^{t}\left(D_{t}\right)$ tal que

$$
d\left(g^{-1}(z), J\right) \geq A / 2 .
$$

Suponhamos o primeiro caso. Lembremos que para $j>T$ o retângulo $D_{j}$ está bem definido e mais ainda $D_{j} \subset V$, o que implica $g(z), g(g(z)), \ldots, g^{t-1-T}(g(z)) \in V$. Assim, usando a 
desigualdade (2.4) temos que

$$
\frac{A}{2} \leq K_{3}\left\|D g_{p}^{-t+T+1} \mid E^{u}\right\|
$$

Por outro lado, usando a desigualdade (2.5) e a vizinhança $\Gamma$, podemos fazer a mesma coisa para o segundo caso, obtendo

$$
\frac{A}{2} \leq K_{5}\left\|D g_{p}^{t-1-T} \mid E^{s}\right\|
$$

E então, como $D g$ é limitado e $T$ é independente de $A$ podemos encontrar $K_{1}$ como afirmado.

Fixemos agora um inteiro positivo grande $n$.

Tomando $\delta>0$ suficientemente pequeno, como $A=\frac{2 K a \delta}{\pi N}$, podemos tomar $N$ grande e usar o Lema 2.3.2 tal que

$$
\frac{1}{t} \log N>\min \left\{\frac{1}{t} \log \left\|D g_{p}^{-t}\left|E^{u}\left\|^{-1}, \frac{1}{t} \log \right\| D g_{p}^{t}\right| E^{s}\right\|^{-1}\right\}-\frac{1}{2 n} .
$$

Observemos que quando $t$ vai para infinito o mínimo acima converge para o mínimo entre o menor expoente de lyapunov positivo, $\chi(p, g)$, e o maior expoente de Lyapunov negativo de $p$ para $g$. No entanto, como estamos no mundo simplético estes dois expoentes coincidem, e assim, desde que $t$ vai para infinito quando $N$ vai, podemos encontrar um inteiro positivo $N_{1}$ tal que

$$
\frac{1}{t} \log N_{1}>\chi(p, g)-\frac{1}{n}
$$

O que implica que é possível encontrar uma $C^{1}$-perturbação $g$ de $f$ tal que

$$
h\left(g \mid \Lambda\left(p, N_{1}\right)\right)>\chi(p, g)-\frac{1}{n} .
$$

No caso geral, quando $p$ não é fixo para $g_{1}$, i.e., $\tau\left(p, g_{1}\right)>1$, a diferença para o caso anterior é que $q \in W_{l o c}^{s}\left(p, g_{1}\right) \cap W^{u}\left(f^{j}(p), g_{1}\right)$, para algum $0 \leq j<\tau\left(p, g_{1}\right)$. Então, como feito anteriormente, podemos encontrar alguma perturbação $g$ de $g_{1}$ e $t=\tau(p, g) \tilde{t}+j$ tal que $g^{t}$ possua um conjunto hiperbólico $\tilde{\Lambda}(p, N)$ como antes. Mais ainda, vai existir uma relação entre a norma de $D g^{\tau(p, g)}$ e $A$ como no Lema 2.3.2, só que neste caso com $\tilde{t}$ ao invés de $t$. Sendo assim, analogamente temos $N_{1}$ tal que

$$
h(g \mid \Lambda(p, N))>\chi(p, g)-\frac{1}{n}, \text { para } N \geq N_{1}
$$

Agora, desde que $\Lambda(p, N)$ é conjugado com o produto entre uma órbita periódica e o shift de $N$ símbolos, existe uma medida ergódica $\mu(N) \in \mathcal{M}(\Lambda(p, N))$ que maximiza a entropia topológica

$$
h_{\mu(N)}(g)>\chi(p, g)-\frac{1}{n}, \text { para } N \geq N_{1} .
$$

Como a argumentação no caso geral $(\tau(p, g)>1)$ é sempre análoga ao feito anteriormente, vamos mostrar as outras propriedades que restam apenas para o caso onde $p$ é um ponto fixo hiperbólico. Continuando então, vamos encontrar um inteiro positivo $N_{2}$ tal que se 
$\mu \in \mathcal{M}\left(f \mid \Lambda\left(g, N_{2}\right)\right)$ é ergódica então $\rho\left(\mu, \mu_{p}\right)<1 / n$, como se pede no item (c) da proposição. Para tal, dado $\zeta>0$ arbitrariamente pequeno é suficiente encontrarmos $N=N(\zeta)$ tal que (a órbita de) todo ponto de $\Lambda(p, N)$ visite muito frequentemente a bola de raio $\zeta$ e centro $p$.

Como $p$ é um ponto fixo hiperbólico temos que

$$
\bigcap_{i \in \mathbb{Z}} g^{i}(V)=\{p\}
$$

Sendo assim, dado $\zeta>0$ arbitrariamente pequeno existe um inteiro positivo $n_{1} \geq T$, dependendo de $\zeta$, tal que para todo $n_{2} \geq n_{1}$

$$
\operatorname{diam} \bigcap_{-n_{2} \leq i \leq n_{2}} g^{i}(V)<\zeta
$$

Agora, se $\bar{V}=\bigcap_{i=0}^{l n_{1}} g^{-i}(V)$ e $z \in \bar{V}$, então para todo $r \in\left[n_{1},(l-1) n_{1}\right)$ temos que

$$
g^{r}(z) \in \bigcap_{|i|<n_{1}} g^{i}(V) \subset B_{\zeta}(p) .
$$

Logo, a fração de tempo em $\left[0, l n_{1}\right)$ que a órbita de $z$ fica em $B_{\zeta}(p)$ é $\frac{l-2}{l}$.

Lembremos que $t$ é o período do conjunto periódico $\Lambda(p, N)$ para $g$, e assim definimos $k=t-T$. Dado $N$ grande o suficiente, seja $l \in \mathbb{N}$ tal que $(l+1) n_{1} \geq k>l n_{1}$. Como para todo $z \in \Lambda(p, N)$ existe $r \in[0, t)$ tal que $g^{r}(z) \in \bar{V}$, a frequência da órbita de $z$ passando em $B_{\zeta}(p)$ é maior do que

$$
\frac{(l-2) n_{1}}{(l+1) n_{1}+T}
$$

Como $l \rightarrow \infty$ quando $N \rightarrow \infty$, dado $\zeta_{1}>0$ existe $N_{2}$ tal que a frequência da órbita de todo ponto $z \in \Lambda(p, N)$ passando em $B_{\zeta}(p)$ é maior do que $1-\zeta_{1}$, e então escolhendo $\zeta_{1}$ menor se necessário temos que

$$
d\left(\mu, \mu_{p}\right)<\frac{1}{n}, \text { para toda medida ergódica } \mu \in \mathcal{M}(\Lambda(p, N)), N \geq N_{2} .
$$

Finalmente, vamos encontrar $N_{3}$ a fim de que a propriedade (d) seja satisfeita para $\Lambda(p, N)$, $N \geq N_{3}$.

Definimos

$$
\begin{gathered}
V_{k}^{u}=V \cap g(V) \cap \ldots \cap g^{k}(V), \mathrm{e} \\
V_{k}^{s}=V \cap g^{-1}(V) \cap \ldots \cap g^{-k}(V) .
\end{gathered}
$$

Dados vetores $v, w \in \mathbb{R}^{2 n}$ e subespaços $E, F \subset \mathbb{R}^{2 n}$ definimos

$$
\begin{gathered}
\operatorname{ang}(v, w)=\left|\tan \left[\arccos \left(\frac{<v, w>}{\|v\|\|w\|}\right)\right]\right|, \\
\operatorname{ang}(v, E)=\min _{w \in E,|w|=1} \operatorname{ang}(v, w) \quad \text { e } \operatorname{ang}(E, F)=\min _{w \in E,|w|=1} \operatorname{ang}(w, F) .
\end{gathered}
$$


30Capítulo 2 - Estimativas para entropia topológica e não existência de extensões simbólicas

Observação 2.3.3. Uma outra definição de ângulo entre dois subespaços na literatura é a seguinte: se $\mathbb{R}^{n}=E \oplus F$ é uma decomposição de $\mathbb{R}^{n}$, seja $L: E^{\perp} \rightarrow E$ a aplicação linear tal que $F=\left\{w+L w ; w \in E^{\perp}\right\}$, então alguns autores definem o ângulo entre $E$ e $F$ como sendo $\|L\|^{-1}$. No entanto, existe uma equivalência entre esta definição e a apresentada aqui.

Precisamos do seguinte lema.

Lema 2.3.4. Com as definiçôes acima, existe constante $K_{6}$ tal que se $z \in V_{k}^{s}, v \in \mathbb{R}^{2 n} \backslash E_{p}^{s}$ e $\operatorname{ang}\left(D g_{p}^{k}(v), E_{p}^{s}\right) \geq 1$, então

$$
\left|D g^{k}(z)(v)\right| \geq K_{6}\left\|D g_{p}^{-k}\right\|^{-1}|v| \min \left\{\operatorname{ang}\left(v, E_{p}^{s}\right), 1\right\}
$$

Demonstração. Usando a decomposição de $\mathbb{R}^{2 n}$ fixada em $V$, temos que $v=\left(v^{s}, v^{u}\right), v^{s(u)} \in$ $E_{p}^{s(u)}$, para todo $v \in \mathbb{R}^{2 n}$. Seja $|v|^{\prime}=\max \left\{\left|v^{s}\right|,\left|v^{u}\right|\right\}$ a norma do máximo.

Como $E_{p}^{s \perp}=E_{p}^{u}$, e $D g^{k}(z)=D g_{p}^{k}$ se $z \in V_{k}^{s}$, temos que

$$
\operatorname{ang}\left(v, E_{p}^{s}\right)=\frac{\left|v^{u}\right|}{\left|v^{s}\right|} \text { e } 1 \leq \operatorname{ang}\left(D g^{k}(z)(v), E_{p}^{s}\right)=\frac{\left|D g_{p}^{k}\left(v^{u}\right)\right|}{\left|D g_{p}^{k}\left(v^{s}\right)\right|}
$$

Então,

$$
\begin{aligned}
\left|D g^{k}(z)(v)\right|^{\prime} & =\left|D g_{p}^{k}\left(v^{u}\right)\right| \\
& \geq\left\|D g_{p}^{-k}\left|E_{p}^{u} \|^{-1}\right| v^{u} \mid\right. \\
& =\left\|D g_{p}^{-k}\left|E_{p}^{u} \|^{-1}\right| v^{s} \mid \operatorname{ang}\left(v, E^{s}\right) ;\right.
\end{aligned}
$$

o que implica

$$
\left|D g^{k}(z)(v)\right|^{\prime} \geq\left\|\left.D g_{p}^{-k}\left|E^{u} \|^{-1}\right| v\right|^{\prime} \min \left\{\operatorname{ang}\left(v, E_{p}^{s}\right), 1\right\} .\right.
$$

Portanto, pela equivalência entre as normas no espaço euclidiano, o resultado segue.

Lembremos agora que

$$
\Lambda(p, N)=\bigcup_{i=0}^{t-1} g^{i}(\tilde{\Lambda}(p, N))
$$

é um conjunto hiperbólico para $g$, onde $g^{i}(\tilde{\Lambda}(p, N)) \subset V$ para $0 \leq i \leq k=t-T$. Mais ainda, por construção de $\tilde{\Lambda}(p, N))$, sabemos que a decomposição hiperbólica $T_{\Lambda(p, N)} M=\tilde{E}^{s} \oplus \tilde{E}^{u}$ é tal que $\tilde{E}^{s}(z)$ e $\tilde{E}^{u}\left(g^{k}(z)\right)$ são próximos de $E_{p}^{s}$ e $E_{p}^{u}$, respectivamente, para todo $z \in \tilde{\Lambda}(p, N)$. Em particular, se $z \in \tilde{\Lambda}(p, N)$ então $\operatorname{ang}\left(D g^{k}(z)(v), E_{p}^{s}\right)>1$ para todo $v \in \tilde{E}^{u}(z)$.

Sendo assim, podemos usar o Lema 2.3.4 para encontrarmos constante $K_{6}$, tal que para todo $z \in \tilde{\Lambda}(p, N)$ e $v \in \tilde{E}^{u}(z)$,

$$
\left|D g^{r}(z)(v)\right| \geq\left(C K_{6}\right)^{l}\left\|D g_{p}^{-k}\right\|^{-l}|v|, \text { para } r=l(k+T), l \in \mathbb{N} .
$$


onde

$$
C=\inf _{z \in V \backslash g^{-1}(V),|v|=1}\left\|D g^{T}(z)(v)\right\| .
$$

Portanto, para $N$ grande o suficiente não é difícil de ver que todos os pontos em $\tilde{\Lambda}(p, N)$ possuem expoentes de lyapunov positivos maiores do que $\chi(p, g)-1 / n$. Em particular, podemos escolher $N_{3}$ tal que $k>>T$, e logo para todo ponto periódico $q \in \Lambda(p, N)$, para $N \geq N_{3}$,

$$
\chi(q, g) \geq \chi(p, g)-\frac{1}{n} .
$$

Sendo assim, tomando $\Lambda(p, n)=\Lambda(p, N)$ para $N=\max \left\{N_{1}, N_{2}, N_{3}\right\}$, as propriedades da proposição são satisfeitas para a perturbação $g$ de $f$.

Agora, pela robustez do ponto periódico hiperbólico $p$ e do conjunto $\Lambda(p, n)$, as propriedades (a) e (b) são satisfeitas para difeomorfismos próximos de $g$ e suas respectivas continuações, desde que $\mu_{n}$ são as medidas ergódicas que maximizam entropia topológica de $g$ restrita a $\Lambda(p, n)$. Notemos que o item (c) depende apenas do tempo de permanência do conjunto $\Lambda(p, n)$ numa vizinhança de $p$, o que também é mantido para perturbações de $g$ e as respectivas continuações de $p$ e $\Lambda(p, g)$. Por fim, é claro que o Lema 2.3.4 ainda é válido para difeomorfismos próximos de $g$, e portanto também temos o item (d). O que então conclui a demonstração da proposição.

\subsection{Exemplos de pontos de descontinuidade para entropia}

Para provarmos o Teorema C, vamos construir um exemplo de um difeomorfismo conservativo $C^{\infty}$ sobre a esfera $S^{2}$ que não seja sequer ponto de semicontinuidade superior para entropia topológica no espaço $\operatorname{Diff}_{m}^{1}\left(S^{2}\right)$.

Seja $S \neq \mathbb{T}^{2}$ uma superfície qualquer. Como $S$ não aceita difeomorfismos de Anosov, pelo Teorema A temos que genericamente para difeomorfismos conservativos sobre $S$

$$
h(f)=s(f) .
$$

Sendo assim, usando que $s($.$) é uma função semicontínua inferior, se encontrarmos um difeo-$ morfismo $f \in \operatorname{Diff}_{m}^{1}\left(S^{2}\right)$ tal que $h(f)<s(f)$, então este é um exemplo onde a entropia topológica não é semicontínua superior.

Para encontrarmos tal difeomorfismo, usamos um notável resultado de Lai-Sang Young [49].

Teorema 2.4.1. Seja $\phi: \mathbb{R} \times M \rightarrow M$ um fluxo numa variedade $M$ 2-dimensional. Então, o difeomorfismo $\phi_{t}=\phi(t,$.$) sobre M$, para todo $t$, possui entropia topológica nula, i.e., $h\left(\phi_{t}\right)=$ 0 .

Sendo assim, por este resultado e a discussão acima, para provarmos o Teorema C é suficiente encontrarmos um fluxo Hamiltoniano em $S^{2}$ possuindo uma órbita periódica hiperbólica. 
No que segue, descrevemos a construção de tal exemplo para o qual usamos o "famoso" pendulum matemático, veja [42]. Lembremos que um campo vetorial Hamiltoniano $X_{H}$ sobre uma variedade simplética $(M, \omega)$ é tal que existe uma aplicação suave $H: M \rightarrow \mathbb{R}$ e

$$
\omega\left(X_{H}, .\right)=d H .
$$

Também, lembremos que $\phi_{t}=\phi(t,):. M \rightarrow M$ é um difeomorfismo simplético, para todo $t \in \mathbb{R}$, onde $\phi$ é o fluxo gerado por $X_{H}$. Notemos que em dimensão dois o espaço dos difeomorfismos conservativos coincide com o espaço dos simpléticos.

Consideremos agora a variedade simplética $\left(S^{2}, \omega\right)$, com $\omega(x)=<x, u \times v>$, para $x \in S^{2} \mathrm{e}$ $u, v \in T_{x} S^{2}$, algum elemento de área induzido sobre $S^{2}$. Se olharmos para $S^{2}$ com coordenadas polares cilíndricas $(\theta, z), 0 \leq \theta<2 \pi$ e $-1<z<1$, numa região longe dos pólos, podemos verificar que $\omega=d \theta \wedge d z$.

Seja $H_{1}(\theta, z)=z$ a função altura sobre a esfera, e $X_{H_{1}}$ o campo vetorial Hamiltoniano gerado por $H_{1}$. Notemos que o fluxo gerado por $X_{H_{1}}$ não possui órbitas periódicas hiperbólicas, pois seus pólos são singularidades não hiperbólicas, e o fluxo longe deles é $\phi(t,(\theta, z))=(\theta+t, z)$, i.e., rotações.

Por outro lado, podemos usar o pendulum matemático sobre $S^{1} \times \mathbb{R}$ para construirmos órbitas periódicas hiperbólicas no fluxo anterior. Seja $H_{2}: S^{1} \times \mathbb{R} \rightarrow \mathbb{R}, H_{2}(\theta, z)=\frac{1}{2} z^{2}-\cos \theta$ a energia total do pendulum, i.e., o campo vetorial Hamiltoniano $X_{H_{2}}$ sobre o cilindro nos dá o retrato de fase do pendulum. Notemos que o fluxo gerado por $X_{H_{2}}$ possui um ponto de equilíbrio instável em $p=(\pi, 0)$. Agora, considerando $\beta:(-1,1) \rightarrow \mathbb{R}$ uma função bump $C^{\infty}$ tal que $\beta(x)=1$ se $|x|<1 / 2$ e $\beta(x)=0$ se $|x|>2 / 3$, definimos $H: S^{1} \times(-1,1) \rightarrow \mathbb{R}$ como segue

$$
H(\theta, z)=\beta(z) H_{2}(\theta, z)+(1-\beta(z)) H_{1}(\theta, z) .
$$

Sendo assim, depois de alguma mudança de coordenada podemos olhar para esta função sobre $S^{2}$. De fato, o que fizemos foi nada mais do que levar o fluxo dado pelo pendulum para a esfera mudando a função altura numa faixa longe dos pólos. Veja figura 2.2.

Agora, $X_{H}$ é um campo vetorial Hamiltoniano sobre $S^{2}$, cujo fluxo possui uma singularidade hiperbólica como queríamos.

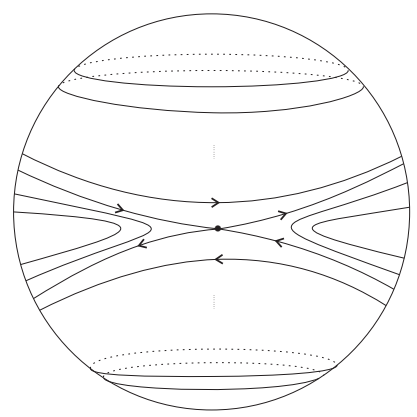

Figura 2.2: Retrato de fase de $X_{H}$ 


$\frac{19}{3}$

\section{Hiperbolicidade no mundo}

conservativo

Neste capítulo, vamos mostrar o Teorema E. Mais precisamente, mostramos que, a menos de uma perturbação, a existência de um ponto periódico não hiperbólico é necessária para que um difeomorfismo $f$ seja não Anosov. Diferentemente da prova de Hayashi [27] para o caso dissipativo, o que fazemos aqui é usar o surgimento de pontos periódicos não hiperbólicos a partir de ciclos heterodimensionais para mostrar que o índice dos pontos periódicos hiperbólicos são constantes para qualquer difeomorfismo numa vizinhança de $f \in \mathcal{G}_{m}^{1}(M)$. Lembrando que $\mathcal{G}_{m}^{1}(M)$ é o conjunto dos difeomorfismos que possuem uma vizinhança na qual todo difeomorfismo possui apenas pontos periódicos hiperbólicos. Depois de feito isto, podemos usar os argumentos de Mañé [35] para provarmos o Teorema E. Por fim, como anunciado na introdução, provamos a Conjectura de Palis no caso conservativo a partir deste.

\section{1 Índice das órbitas periódicas para difeomorfismos em $\mathcal{G}_{m}^{1}(M)$}

Antes de mais nada, definimos como sendo o indice de um ponto periódico hiperbólico a dimensão da sua variedade estável. Como antes $M$ é uma variedade Riemanianna, compacta e conexa, porém agora com dimensão $d \geq 2$ qualquer, e munida de uma forma de volume $m$. O resultado seguinte diz que a existência de selas hiperbólicas com índices diferentes não pode ocorrer se $f \in \mathcal{G}_{m}^{1}(M)$. Mais precisamente mostramos o seguinte. 
Proposição 3.1.1. Seja $f \in \mathcal{G}_{m}^{1}(M)$, então existe uma vizinhança $\mathcal{U}$ de $f$ em $\operatorname{Diff}_{m}^{1}(M) e$ um inteiro positivo $i$ tal que para todo difeomorfismo $g \in \mathcal{U}$ e todo ponto periódico hiperbólico $p$ de $g$, o indice de $p$ com respeito a $g$ é $i$.

Este resultado pode ser visto como a versão conservativa e discreta do resultado de Gan e Wen (veja Teorema 4.1 de [25]) desde que a demonstração do mesmo é feita através da criação de ciclos heterodimensionais. Dizemos que um difeomorfismo $f$ exibe um ciclo heterodimensional se existem pontos periódicos hiperbólicos $p_{1}$ e $p_{2}$ de $f$ com índices diferentes, digamos $i$ e $i+j$, possuindo uma interseção transversal entre $W^{u}\left(p_{1}, f\right)$ e $W^{s}\left(p_{2}, f\right)$, e uma interseção quasi-transversal entre $W^{s}\left(p_{1}, f\right)$ e $W^{u}\left(p_{2}, f\right)$. Veja Figura 3.1.

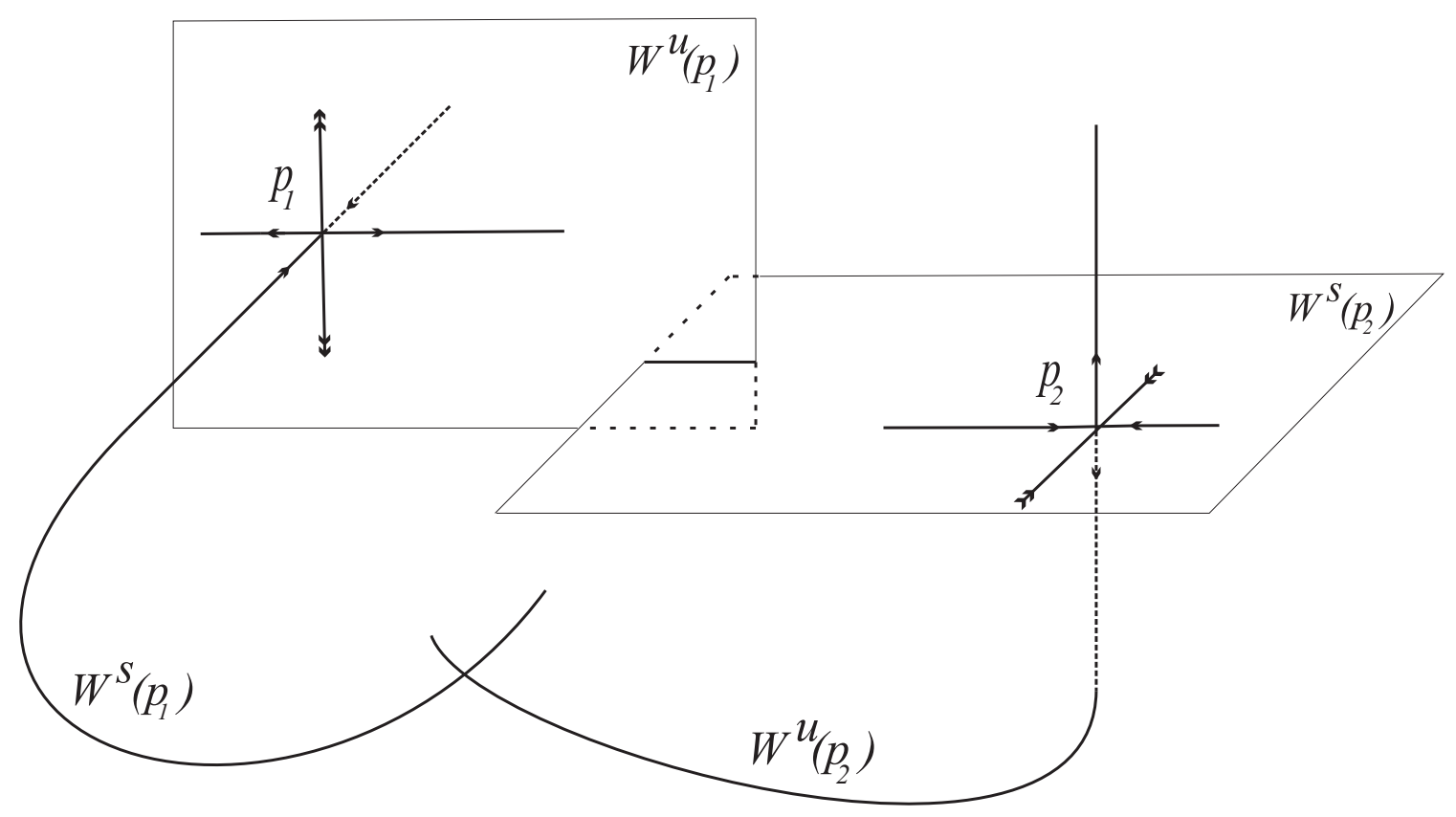

Figura 3.1: ciclo heterodimensional

O próximo resultado é chave na demonstração da Proposição 3.1.1.

Proposição 3.1.2. Seja $f \in \operatorname{Diff}_{m}^{1}(M)$ um difeomorfismo exibindo um ciclo heterodimensional entre dois pontos periódicos hiperbólicos $p_{1}$ e $p_{2}$ com indices $i$ e $i+j$, respectivamente, tal que $D f_{p_{1}}^{\tau\left(p_{1}, f\right)}$ e $D f_{p_{2}}^{\tau\left(p_{2}, f\right)}$ possuam apenas autovalores reais com multiplicidade um. Dado uma vizinhança qualquer $\mathcal{U}$ de $f$ em $\operatorname{Diff}_{m}^{1}(M)$, existe um difeomorfismo $g \in \mathcal{U}$ possuindo um ponto periódico p não hiperbólico.

Observação 3.1.3. A Proposição 3.1.2 segue nas linhas do trabalho de Abdenur-BonattiCrovisier-Diaz-Wen [2], i.e., a ideia de usar ciclos heterodimensionais para se construir pontos periódicos com exponentes de Lyapunov intermediários com respeito aos expoentes dos pontos periódicos hiperbólicos que exibem o ciclo. Convém assim ressaltarmos que usando as técni- 
cas apresentadas aqui a menos de algumas adaptaçôes e argumentos padrões para se provar generecidade, podemos obter uma prova do resultado deles no caso conservativo.

Vejamos agora a demonstração da Proposição 3.1.1 usando a Proposição 3.1.2.

Demonstração Proposição 3.1.1:

Suponhamos por absurdo que existam dois pontos periódicos hiperbólicos $\tilde{p}_{1}$ e $\tilde{p}_{2}$ de $f \in$ $\mathcal{G}_{m}^{1}(M)$ com índices $i$ e $i+j$, para algum $j>0$.

A ideia é usar a Proposição 3.1.2, para chegarmos numa contradição e assim provarmos a proposição.

Para tal, os dois próximos resultados são essenciais.

Proposição 3.1.4 (Bonatti e Crovisier [9]). Existe um subconjunto residual $\mathcal{R}_{1}$ de $\operatorname{Diff}_{m}^{1}(M)$ tal que se $g \in \mathcal{R}$ então $M=\overline{H(p, g)}$, onde $\overline{H(p, g)}$ é a classe homoclínica do ponto periódico hiperbólico $p$ de $g$. Em particular, g é topologicamente transitivo.

Proposição 3.1.5 (Carballo, Morales e Pacífico [18]). Existe um subconjunto residual $\mathcal{R}_{2} \in$ $\operatorname{Diff}_{m}^{1}(M)$ tal que se $f \in \mathcal{R}_{2}$ então as classes homoclínicas ou são iguais ou são disjuntas.

O primeiro passo será perturbar $f$ a fim de obtermos pontos periódicos hiperbólicos com índices $i$ e $i+j$ como na hipótese da Proposição 3.1.2. Para tal, usamos os resultados de Bonatti, Diaz e Pujals em [10], para sistemas lineares periódicos (cociclos).

Um sistema linear periódico é uma 4-upla $\mathcal{P}=(\Sigma, f, \mathcal{E}, A)$, onde $f$ é um difeomorfismo, $\Sigma$ é um conjunto infinito de pontos periódicos de $f, \mathcal{E}$ um fibrado vetorial Euclidiano sobre $\Sigma$, e $A \in G L(\Sigma, f, \mathcal{E})$ é tal que $A(x): \mathcal{E}_{x} \rightarrow \mathcal{E}_{f(x)}$ é um isomorfismo linear para cada $x$ ( $\mathcal{E}_{x}$ é a fibra de $\mathcal{E}$ em $x$ ). Para definições mais precisas veja [10]. Lembremos que dois pontos periódicos hiperbólicos possuindo índices iguais são homoclinicamente relacionados se a variedade estável de um intersecta transversalmente a variedade instável do outro, e vice-versa.

Lema 3.1.6 (Lema $1.9 \mathrm{em} \mathrm{[10]).} \mathrm{Seja} \overline{H(p, f)}$ uma classe homoclínica não trivial. Então a derivada $D f$ de $f$ induz um sistema linear periódico com transições sobre $\operatorname{Per}_{h}(\overline{H(p, f)})$, o conjunto dos pontos periódicos hiperbólicos homoclinicamente relacionados com $p$.

Dizemos que um sistema linear periódico com transições $\mathcal{P}=(\Sigma, f, \mathcal{E}, A)$ é diagonalizável no ponto $x \in \Sigma$ se a aplicação linear

$$
M_{A}(x): \mathcal{E}_{x} \rightarrow \mathcal{E}_{x}, \quad M_{A}(x)=A\left(f^{\tau(x, f)-1}(x)\right) \circ \ldots \circ A\left(f^{2}(x)\right) \circ A(x),
$$

possui apenas autovalores reais com multiplicidade um.

Lema 3.1.7 (Lema 4.16 em [10]). Para todo sistema linear periódico com transiçôes $\mathcal{P}=$ $(\Sigma, f, \mathcal{E}, A)$ e todo $\varepsilon>0$ existe um subconjunto denso $\Sigma^{\prime}$ de $\Sigma$ e uma $\varepsilon$-perturbação $A^{\prime}$ de $A$ definida em $\Sigma^{\prime}$ que é diagonalizável, isto é, $M_{A^{\prime}}(x)$ possui apenas autovalores reais com multiplicidade um para todo $x \in \Sigma^{\prime}$. 
Observação 3.1.8. Pela observação $7.2 \mathrm{em}$ [10], se as matrizes A possuem determinante 1 (que é o caso do sistema linear periódico induzido por $f \in \operatorname{Diff}_{m}^{1}(M)$ ) podemos tomar a perturbação $A^{\prime}$ tal que $\operatorname{det} A^{\prime}(x)=1$ para todo $x \in \Sigma^{\prime}$.

Agora, usando as Proposições 3.1.4 e 3.1.5 podemos supor inicialmente que $f \in \mathcal{R}_{1} \cap$ $\mathcal{R}_{2}$, logo $\overline{H\left(\tilde{p}_{1}, f\right)}=\overline{H\left(\tilde{p}_{2}, f\right)}=M$. Sendo assim, desde que as classes homoclínicas são não triviais, podemos usar os Lemas 3.1.6 e 3.1.7, juntamente com a observação 3.1.8 para encontrar $p_{1}$ e $p_{2}$ homoclinicamente relacionados com $\tilde{p}_{1}$ e $\tilde{p}_{2}$, respectivamente, e matrizes $A^{\prime}$ com determinantes um, tal que $M_{A^{\prime}}\left(p_{1}\right)$ e $M_{A^{\prime}}\left(p_{2}\right)$ sejam diagonalizáveis. Desta forma, podemos usar o lema de Franks para perturbar $f$ tal que $D f_{p_{1}}^{\tau\left(p_{1}, f\right)}=M_{A^{\prime}}\left(p_{1}\right)$ e $D f_{p_{2}}^{\tau\left(p_{2}, f\right)}=$ $M_{A^{\prime}}\left(p_{2}\right)$, ou seja, $D f_{p_{1}}^{\tau\left(p_{1}, f\right)}$ e $D f_{p_{2}}^{\tau\left(p_{2}, f\right)}$ possuem apenas autovalores reais com multiplicidade um.

O próximo passo é perturbar $f$ a fim de exibirmos um ciclo heterodimensional entre $p_{1} \mathrm{e}$ $p_{2}$.

Usando a robustez de $p_{1}$ e $p_{2}$ e de suas propriedades, podemos supor sem perda de generalidade que o difeomorfismo inicial $f \in \mathcal{R}_{1}$ e portanto é transitivo. Com isto, podemos usar o connecting lema para criar uma interseção entre $W^{u}\left(p_{1}, f\right)$ e $W^{s}\left(p_{2}, f\right)$, a qual podemos assumir ser transversal $\left(\operatorname{dim} W^{u}(p, f)+\operatorname{dim} W^{s}(p, f)>d\right)$. Sendo assim, desde que esta interseção é robusta, podemos supor que $f$ ainda pertença a $\mathcal{R}_{1}$, i.e., ainda seja transitivo. Por fim, usamos mais uma vez o conecting lema a fim de conseguirmos uma interseção entre $W^{s}\left(p_{1}, f\right)$ e $W^{u}\left(p_{2}, f\right)$. Logo, criamos um ciclo heterodimensional para $f$ entre os pontos $p_{1}$ e $p_{2}$ após uma perturbação.

Assim, temos um difeomorfismo $g C^{1}$-próximo de $f$ exibindo um ciclo heterodimensional entre os pontos periódicos hiperbólicos $p_{1}$ e $p_{2}$, com $D g^{\tau\left(p_{1}, g\right)}\left(p_{1}\right)$ e $D g^{\tau\left(p_{2}, g\right)}\left(p_{2}\right)$ possuindo apenas autovalores reais com multiplicidade um. Portanto, pela Proposição 3.1.2 podemos perturbar $g$ tal que esta possua um ponto periódico não hiperbólico, o que contradiz o fato de $f \in \mathcal{G}_{m}^{1}(M)$ e portanto prova a proposição.

Antes de provarmos a Proposição 3.1.2, precisamos de algumas definições.

Dizemos que um conjunto compacto $f$-invariant $\Lambda$ possui uma decomposição dominada se existe uma decomposição contínua $T_{\Lambda} M=E_{1} \oplus \ldots \oplus E_{k} D f$-invariante, e constantes $m \in \mathbb{N}$ e $0<\lambda<1$ tal que para todo $x \in \Lambda$ tenhamos:

$$
\left\|D f^{m}\left|E_{i}(x)\|\| D f^{-m}\right| E_{j}\left(f^{m}(x)\right)\right\| \leq \lambda, \text { para todo } i<j .
$$

\section{Demonstração da Proposição 3.1.2:}

Seja $f \in \operatorname{Diff}_{m}^{1}(M)$ satisfazendo as hipóteses da proposição, e sejam $x \in W^{s}\left(p_{1}, f\right) \cap$ $W^{u}\left(p_{2}, f\right)$ e $y \in W^{u}\left(p_{1}, f\right) \cap W^{s}\left(p_{2}, f\right)$ pontos heteroclínicos do ciclo. Agora, desde que os pontos periódicos são hiperbólicos, por Avila [6] podemos assumir que o difeomorfismo $f$ seja $C^{2}$, e assim podemos usar o pasting lema para linearizarmos o difeomorfismo numa vizinhança de $p_{1}$ e $p_{2}$, i.e., $f^{\tau\left(p_{k}, f\right)}=D f_{p_{k}}^{\tau\left(p_{k}, f\right)}$ em coordenadas locais $\left(U_{k}, \phi_{k}\right), k=1,2$, onde $U_{1}$ e $U_{2}$ 
são vizinhanças de $p_{1}$ e $p_{2}$, respectivamente. É importante observar que esta perturbação mantém a função igual no complementar de $U_{1}$ e $U_{2}$. Agora, apesar de termos uma mudança das variedades estável e instável de $p_{1}$ e $p_{2}$, esta variação é contínua para partes compactas destas variedades, e assim usando o Lema 1.2 .1 podemos conectar novamente $W^{s}\left(p_{1}, f\right)$ e $W^{u}\left(p_{2}, f\right)$, numa vizinhança de $x$, por uma perturbação pequena. Note que a outra interseção é transversal, logo não é quebrada por perturbações. Portanto, a menos de uma perturbação, podemos supor que $f$ seja linear (em coordenadas locais) em vizinhanças arbitrariamente pequenas $U_{1}$ e $U_{2}$ de $p_{1}$ e $p_{2}$, respectivamente, e ainda exiba um ciclo heterodimensional.

A fim de não complicar a notação, assumimos $p_{1}$ e $p_{2}$ como pontos fixos. O caso geral é inteiramente análogo. A partir de agora também olhamos $U_{1}$ e $U_{2}$ em coordenadas locais.

Desde que temos apenas autovalores reais com multiplicidade um para $D f_{p_{1}}$ e $D f_{p_{2}}$, a menos de uma mudança de coordenadas, temos a seguinte decomposição ortonormal em $U_{1} \cap f^{-1}\left(U_{1}\right)$ e $U_{2} \cap f^{-1}\left(U_{2}\right)$ por subespaços invariantes $E_{1} \oplus \ldots \oplus E_{d}(\operatorname{dim} M=d)$. Mais ainda, denotando por $\lambda_{k}$ e $\sigma_{k}, k=0, \ldots, d$, os autovalores ordenados de $p_{1}$ e $p_{2}$, respectivamente, estes subespaços são os autoespaços correspondentes. Observemos assim que a variedade estável e instável de $p_{1}$ são respectivamente $E_{p_{1}}^{s}=E_{1} \oplus \ldots \oplus E_{i}$ e $E_{p_{1}}^{u}=E_{i+1} \oplus \ldots \oplus E_{d}$, enquanto que a variedade estável e instável de $p_{2}$ são $E_{p_{2}}^{s}=E_{1} \oplus \ldots \oplus E_{i+j}$ e $E_{p_{2}}^{u}=E_{i+j+1} \oplus$ $\ldots \oplus E_{d}$, respectivamente. Note que estas decomposições são dominadas.

O próximo passo consiste em fazer uma série de perturbações de tal forma a conseguirmos uma decomposição dominada para o conjunto f-invariante $O(x) \cup O(y) \cup\left\{p_{1}, p_{2}\right\}$, onde $O(x)$ e $O(y)$ são as órbitas de $x$ e $y$, respectivamente.

Como antes, seja $x$ um ponto de interseção entre $W^{s}\left(p_{1}, f\right)$ e $W^{u}\left(p_{2}, f\right)$ e $y$ um ponto de interseção entre $W^{u}\left(p_{1}, f\right)$ e $W^{s}\left(p_{2}, f\right)$. Consideremos agora o subespaço $E=T_{y}\left(W^{u}\left(p_{1}, f\right) \cap\right.$ $\left.W^{s}\left(p_{2}, f\right)\right)$ de dimensão $j$. Desde que $y$ pertence a variedade instável do ponto $p_{1}$, e $f$ é linear em $U_{1}$, temos que $D f_{y}^{-n}(E) \subset E_{p_{1}}^{u}$ para valores grandes de $n$. A menos de uma perturbação (usando transversalidade), podemos supor que $D f_{y}^{-n}(E) \cap E_{i+j+1} \oplus \ldots \oplus E_{d}=\{0\}$, e assim usando o fato da decomposição em $U_{1}$ ser dominada, temos que $D f_{y}^{-n}(E) \rightarrow E_{i+1} \oplus \ldots \oplus E_{i+j}$ quando $n \rightarrow \infty$. Escolhendo $n$ grande o suficiente podemos fazer uma perturbação de $f$, usando o lema de Franks, de tal forma que $D f_{y}^{-n}(E)=E_{i+1} \oplus \ldots \oplus E_{i+j}$. Note que esta perturbação é local, mais precisamente, a função $f$ é alterada apenas numa vizinhança de $f^{-n+1}(y)$, e além disto não altera a órbita de $y$, o que implica que $y$ continua a ser um ponto de interseção heteroclínica. Com os mesmos argumentos, só que agora olhando para órbita futura de $y$, podemos fazer uma perturbação de $f$ tal que $D f_{y}^{n}(E)=E_{i+1} \oplus \ldots \oplus E_{i+j} \subset E_{p_{2}}^{s}$.

Prosseguindo, seja $E_{p_{1}}^{u u}=E_{i+j+1} \oplus \ldots \oplus E_{d}$. Usando $\lambda$-lema temos que $D f_{f_{y}^{-n}}^{m}\left(E_{p_{1}}^{u u}\right)$ converge a $E_{p_{2}}^{u}$ quando $m \rightarrow \infty$, e assim, podemos fazer uma nova perturbação usando o lema de Franks mantendo invariante a direção $E$, porém fazendo com que $D f_{f_{y}^{-n}}^{m}\left(E_{p_{1}}^{u u}\right)=E_{p_{2}}^{u}$. Da mesma forma, podemos fazer com que $D f_{f_{y}^{n}}^{-m}\left(E_{p_{2}}^{s s}\right)=E_{p_{1}}^{s}$, mantendo invariante as direções $E$ e $E^{u u}$, onde $E_{p_{2}}^{s s}=E_{1} \oplus \ldots \oplus E_{i}$. 
Repetindo agora este processo dentro de cada uma das direções invariantes obtidas acima, podemos perturbar $f$ tal que

$$
D f_{f^{-n}(y)}^{2 n}\left(E_{k}\right)=E_{k}, k=0, \ldots, d \text { e } n \text { suficientemente grande } .
$$

Completamente análogo, só que agora usando as variedades centro estável e centro instável no ponto $x\left(E_{p_{1}}^{c s}=E_{1} \oplus \ldots \oplus E_{i+j}\right.$ e $\left.E_{p_{2}}^{c u}=E_{i+1} \oplus \ldots \oplus E_{d}\right)$ podemos perturbar $f$ para que também tenhamos

$$
D f_{f^{-n}(x)}^{2 n}\left(E_{k}\right)=E_{k}, k=0, \ldots, d \text { e } n \text { suficientemente grande } .
$$

Sendo assim, temos uma decomposição dominada $T_{\Lambda} M=E_{1} \oplus \ldots \oplus E_{d}$ para o conjunto f-invariante $\Lambda=O(x) \cup O(y) \cup\left\{p_{1}, p_{2}\right\}$. Logo, se $U$ é uma vizinhança pequena de $\Lambda$, o conjunto maximal invariante em $U$ para $g$ suficientemente próximo de $f$,

$$
\Lambda_{U}(g)=\bigcap_{-\infty}^{+\infty} g^{k}(U),
$$

possui uma decomposição dominada por subfibrados unidimensionais assim como $\Lambda$ para $f$.

Afirmação: Existe um difeomorfismo $g \in \mathcal{U}$ possuindo um ponto periódico hiperbólico $p$ com um autovalor tão próximo de um quanto se queira.

Para tal fixemos inicialmente $\varepsilon_{0}>0$ e $c>0$ para $f$ de acordo com o Lema 1.2.1, e seja $0<\varepsilon<\varepsilon_{0}$ tal que se $f_{1} \in \operatorname{Diff}_{m}^{1}(M)$ é $\varepsilon-C^{1}$ próximo de $f$ então $f_{1} \in \mathcal{U}$.

Tomemos agora, $B_{p_{1}} \subset U_{1} \cap U$ e $B_{p_{2}} \subset U_{2} \cap U$ bolas pequenas centradas em $p_{1}$ e $p_{2}$, respectivamente, onde $U_{1}$ e $U_{2}$ podem ser tomadas menores tal que $f$ ainda seja linear (em coordenadas locais) nestas vizinhanças, como antes. Por escolha de $x$ e $y$, podemos tomar inteiros positivos $m_{1}, m_{2}, m_{3}$ e $m_{4}$ tal que $x^{+}=f^{m_{1}}(x), y^{-}=f^{-m_{3}}(y) \in B_{p_{1}}$ e $x^{-}=$ $f^{-m_{2}}(x), y^{+}=f^{m_{4}}(y) \in B_{p_{2}}$. Feito isto, seja $0<\delta<\varepsilon_{0}$ tal que

$$
f^{-1}\left(B_{\delta}\left(x^{+}\right)\right) \cap B_{\delta}\left(x^{+}\right)=\varnothing, f^{-1}\left(B_{\delta}\left(f\left(x^{-}\right)\right)\right) \cap B_{\delta}\left(f\left(x^{-}\right)\right)=\varnothing,
$$

e

$$
f^{-1}\left(B_{\delta}\left(y^{+}\right) \cap B_{\delta}\left(y^{+}\right)=\varnothing, f^{-1}\left(B_{\delta}\left(f\left(y^{-}\right)\right)\right) \cap B_{\delta}\left(f\left(y^{-}\right)\right)=\varnothing .\right.
$$

Através do $\lambda$-Lema, podemos encontrar $z_{m} c \delta \varepsilon$-próximo de $x^{+}$tal que $f^{m}\left(z_{m}\right)$ seja também $c \delta \varepsilon$-próximo de $y^{-}$e $f^{r}\left(z_{m}\right) \in B_{p_{1}}, 0 \leq r \leq m$, para todo $m>0$ suficientemente grande. Analogamente, podemos encontrar $\bar{z}_{n}$ satisfazendo condições similares quando trocamos $p_{1}$ por $p_{2}, x^{+}$por $x^{-}$e $y^{-}$por $y^{+}$. Veja a figura 3.2 .

Sendo assim, o conjunto

$$
O_{m n}=\left\{z_{m}, \ldots, f^{m}\left(z_{m}\right), y^{-}, \ldots, y^{+}, \bar{z}_{n}, \ldots, f^{n}\left(\bar{z}_{n}\right), x^{-}, \ldots, x^{+}\right\}
$$

é uma pseudo órbita periódica. Usando o Lema 1.2.1, podemos perturbar $f$ a fim de obtermos de fato uma órbita periódica $p_{m n}$ que sombreie $O_{m n}$. Mais ainda, isto é feito tal que

$$
\left\{z_{m}, \ldots, f^{m-1}\left(z_{m}\right), y^{-}, \ldots, f^{-1}\left(y^{+}\right), \bar{z}_{n}, \ldots, f^{n-1}\left(\bar{z}_{n}\right), x^{-}, \ldots, f^{-1}\left(x^{+}\right)\right\}
$$




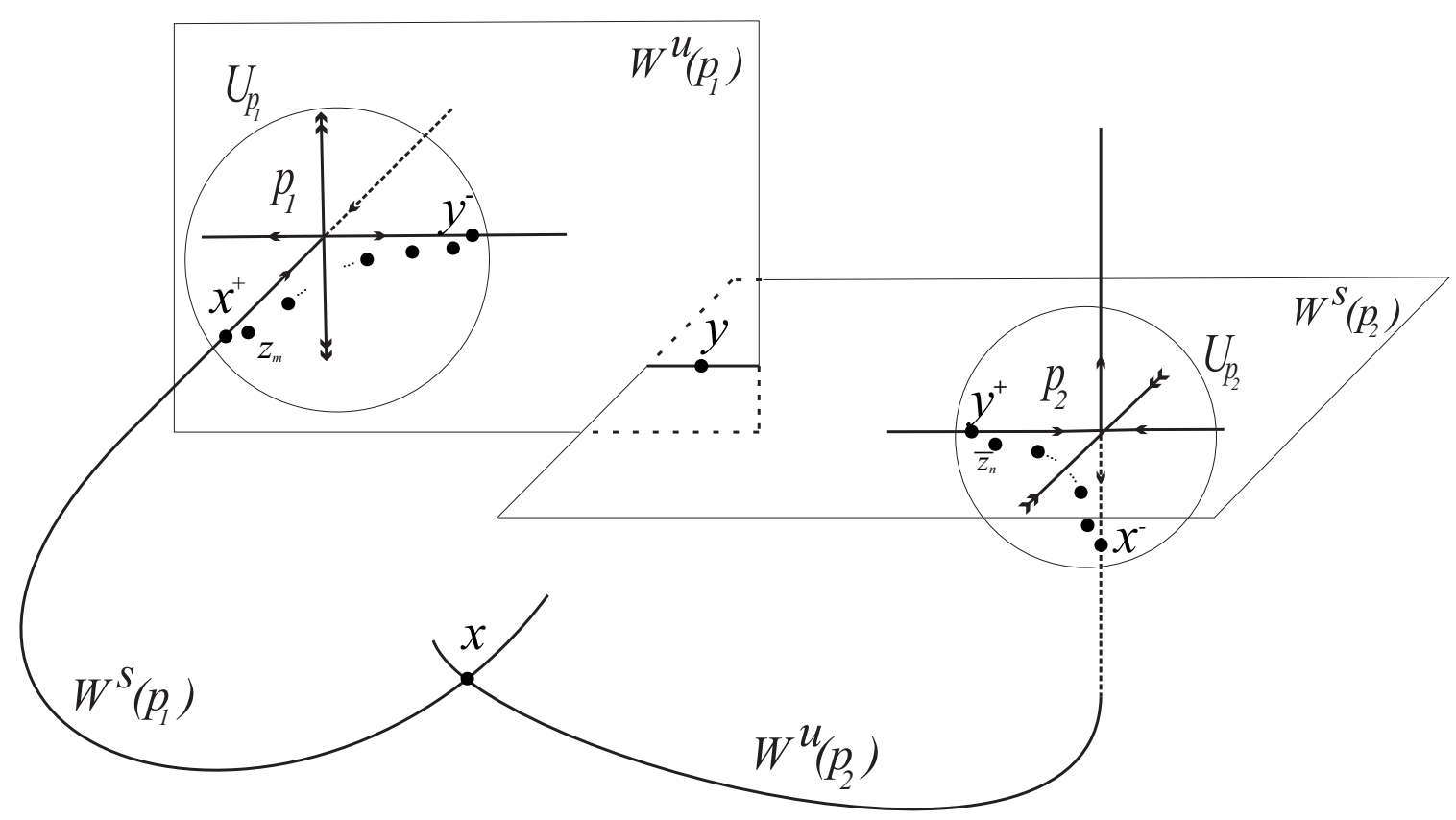

Figura 3.2:

seja a órbita do ponto periódico $p_{m n}$. Observe que a órbita de $p_{m n}$ passa $m$ e $n$ vezes em $B_{p_{1}}$ e $B_{p_{2}}$, respectivamente. Além do mais, por construção, temos que $p_{m n} \in U$, logo $p_{m n} \in \Lambda_{U}(f)$. Denotando agora por $\rho_{k}$ os autovalores de $D f_{p_{m n}}^{\tau\left(p_{m n}, f\right)}$, como temos uma decomposição dominada em $\Lambda_{U}(f)$ por subfibrados unitários e $p_{m n}$ pertence a este conjunto, temos que os $E_{k}\left(p_{m n}\right), k=0, \ldots, d$, são de fato os autoespaços correspondentes a estes autovalores. Fixemos então algum $k \in(i, i+j)$. Lembremos que para tal $k$, os autovalores $\lambda_{k}$ e $\sigma_{k}$ de $D f_{p_{1}}$ e $D f_{p_{2}}$, respectivamente, são tais que $\left|\lambda_{k}\right|>1$ e $\left|\sigma_{k}\right|<1$. Sendo assim, de acordo com o tempo de permanência do ponto $p_{m n}$ nas vizinhanças $B_{p_{1}}$ e $B_{p_{2}}$ que é $m$ e $n$, respectivamente, o valor absoluto do autovalor $\rho_{k}$ de $p_{m n}$, com respeito ao autoespaço $E_{k}$, pode ser tanto maior ou menor do que um.

Dado $n$ um inteiro positivo grande, escolhemos $m=m(n)$ o maior inteiro positivo possível tal que os pontos periódicos $p_{m-1 n}$ e $p_{m n}$, obtidos pelos métodos acima, possuam autovalores na direção $E_{k}$ contrator e expansor, respectivamente. Mais precisamente, se $h$ e $g$ são as perturbações de $f$ tal que $p_{m-1 n} \in \operatorname{Per}(h)$ e $p_{m n} \in \operatorname{Per}(g)$, então $m$ é escolhido tal que $\left\|D h^{\tau\left(p_{m-1 n}, h\right)} \mid E_{k}\left(p_{m-1 n}\right)\right\|<1$ e $\left\|D g^{\tau\left(p_{m n}, g\right)} \mid E_{k}\left(p_{m n}\right)\right\|>1$. Observemos que a maneira como perturbamos $f$ para chegarmos a $h$ e $g$ é tal que $h=g$ no complementar de $B_{p_{1}}$, e $h=g=f$ no complementar de $B_{p_{1}} \cup B_{p_{2}}$.

Como já observado anteriormente, a órbita dos pontos periódicos $p_{l n}, l=m-1, m$, é

$$
\left\{z_{l}, \ldots, f^{l-1}\left(z_{l}\right), y^{-}, \ldots, f^{-1}\left(y^{+}\right), \bar{z}_{n}, \ldots, f^{n-1}\left(\bar{z}_{n}\right), x^{-}, \ldots, f^{-1}\left(x^{+}\right)\right\}
$$


para $l=m-1, m$, onde $z_{l}$ e $\bar{z}_{n}$ podem ser encontrados através do $\lambda$-lemma, dependendo de $l$, como antes.

Agora, dado $\gamma>0$ arbitrariamente pequeno, podemos tomar $n$ maior se necessário tal que denotando por $\tau=\tau\left(y^{-}, g\right)$ o período de $y^{-} \in O\left(p_{m n}\right)$ e tomando $K=\left\|D f \mid E_{k}\left(p_{1}\right)\right\|$, temos que

$$
\begin{aligned}
0 & <\frac{1}{\tau} \log \left\|D g^{\tau}\left|E_{k}\left(y^{-}\right)\left\|=\frac{1}{\tau} \sum_{t=0}^{\tau-1} \log \right\| D g\right| E_{k}\left(g^{t}\left(y^{-}\right)\right)\right\| \\
& <\frac{1}{\tau}\left(\sum_{t=0}^{\tau-1} \log \left\|D f \mid E_{k}\left(g^{t}\left(y^{-}\right)\right)\right\|+\gamma\right) \\
& \leq \frac{1}{\tau}\left(\sum_{t=0}^{\tau-m-1} \log \left\|D f \mid E_{k}\left(g^{t}\left(y^{-}\right)\right)\right\|+m\left(\log \left\|D f \mid E_{k}\left(p_{1}\right)\right\|+\gamma\right)+\gamma \tau\right) \\
& <\frac{1}{\tau-1}\left(\sum_{t=0}^{\tau-m-1} \log \left\|D f \mid E_{k}\left(g^{t}\left(y^{-}\right)\right)\right\|+(m-1)\left(\log \left\|D f \mid E_{k}\left(p_{1}\right)\right\|\right)\right)+2 \gamma+\frac{K}{\tau},
\end{aligned}
$$

onde usamos que a direção $E_{k}$ é unidimensional na primeira igualdade, a continuidade da decomposição dominada na segunda desigualdade, e o fato de $B_{p_{1}}$ ser pequena na terceira. Por outro lado, usando o ponto periódico $y^{-} \in O\left(p_{m-1 n}\right)$ de $h$, com período $\tau-1$, através de argumentos similares temos o seguinte:

$$
\begin{aligned}
0 & >\frac{1}{\tau-1} \log \left\|D h ^ { \tau - 1 } | E _ { k } ( y ^ { - } ) \| = \frac { 1 } { \tau - 1 } \sum _ { t = 0 } ^ { \tau - 1 } \operatorname { l o g } \| D h | E _ { k } \left(h^{t}\left(y^{-}\right) \|\right.\right. \\
& >\frac{1}{\tau-1}\left(\sum_{t=0}^{\tau-1} \log \left\|D f \mid E_{k}\left(h^{t}\left(y^{-}\right)\right)\right\|-\gamma\right) \\
& >\frac{1}{\tau-1}\left(\sum_{t=0}^{\tau-m-1} \log \| D f \mid E_{k}\left(h^{t}\left(y^{-}\right) \|+(m-1)\left(\log \left\|D f \mid E_{k}\left(p_{1}\right)\right\|\right)\right)-2 \gamma\right.
\end{aligned}
$$

Agora, como $g=h$ no complementar de $B_{p_{1}}$ e o pedaço de órbita de $y^{-}$por $g$ e $h$ também coincidem no complementar de $B_{p_{1}}$, isto é, temos que $g^{t}\left(y^{-}\right)=h^{t}\left(y^{-}\right)$para $0 \leq t \leq \tau-m-1$. Sendo assim, podemos substituir a desigualdade (3.2) em (3.1), para obtermos o seguinte

$$
0<\frac{1}{\tau} \log \left\|D g^{\tau} \mid E_{k}\left(y^{-}\right)\right\|<4 \gamma+\frac{K}{\tau}
$$

Portanto, desde que o período $\tau$ vai para infinito quando $n$ vai para infinito, e $\gamma>0$ é arbitrário, é possível encontrarmos um ponto periódico $p_{m n}$ para uma perturbação $g$ de $f$ com expoente de Lyapunov suficientemente próximo de zero na direção $E_{k}$, ou seja, $D g_{p_{m n}}^{\tau}$ possui um autovalor suficientemente próximo de um. O que prova a afirmação.

No caso geral, quando $p_{1}$ e $p_{2}$ são pontos periódicos hiperbólicos, o processo é inteiramente análogo. A diferença é que tomamos vizinhanças das órbitas de $p_{1}$ e $p_{2}$, e os números $m$ e $n$ são múltiplos dos períodos de $p_{1}$ e $p_{2}$, respectivamente. 
Finalmente, sejam $\mathcal{U}_{0} \subset \mathcal{U}$ e $\delta>0$ dados pelo lema de Franks para o difeomorfismo $f$. Usando a Afirmação, podemos encontrar um difeomorfismo $\tilde{g} \in \mathcal{U}_{0}$ possuindo um ponto periódico $p$ com período $\tau=\tau(p, \tilde{g})$ grande, e um autovalor $\rho$ de $D \tilde{g}_{p}^{\tau}$ tal que $|\rho|=1 / \alpha$ para $\alpha$ muito perto de um. Agora, denotando o autoespaço correspondente a este autovalor por $E_{k}$ podemos usar o lema de Franks para $\tilde{g}$ na órbita do ponto periódico para encontrarmos um difeomorfismo $g \in \mathcal{U}$ tal que $D g_{\tilde{g}^{t}\left(p_{m n}\right)}\left(v_{t}\right)=(\alpha)^{\frac{1}{\tau}} D \tilde{g}_{\tilde{g}^{t}\left(p_{m n}\right)}\left(v_{t}\right), v_{t} \in E_{k}\left(\tilde{g}^{t}(p)\right)$ unitário, e $p_{m n}$ ainda seja um ponto periódico para $g$. Assim, desde que o espaço $E_{k}$ é unidimensional, como podemos ver na demonstração da Afirmação, temos que o autovalor de $D g_{p_{m n}}^{\tau}$ na direção $E_{k}$ tem valor absoluto igual a um, ou seja, $p_{m n}$ é um ponto periódico não hiperbólico para $g$, como queríamos.

\subsection{Demonstração do Teorema E}

Seguimos aqui argumentos similares a demonstração do Teorema B de Mañé em [35]. Sendo assim, dividimos a prova em dois passos.

Se $f \in \mathcal{G}_{m}^{1}(M)$, então

- Passo 1: $\overline{\operatorname{Per}(f)}$ é um conjunto hiperbólico para $f$,

- Passo 2: $\Omega(f)=\overline{\operatorname{Per}(f)}$.

A partir disto temos que $f$ é Anosov desde que $\Omega(f)=M$ pelo Teorema de Recorrência de Poincaré, o que prova o Teorema E.

\section{Demonstração do Passo 1:}

Para isto, precisamos de um resultado que é a versão conservativa do resultado de Mañé, Proposição II.1 de [35] (veja também o trabalho de Liao [33]). Denotando por $\Lambda_{i}(f)$ o conjunto dos pontos periódicos hiperbólicos de $f$ com índice $i$ o resultado é o seguinte.

Proposição 3.2.1. Se $f \in \mathcal{G}_{m}^{1}(M)$, então existe uma vizinhança $\mathcal{U}$ de $f$ em $\operatorname{Diff}_{m}^{1}(M)$, e constantes $K>0, m \in \mathbb{N}$ e $0<\lambda<1$ tal que

a) Para todo $g \in \mathcal{U}$ e $p \in \operatorname{Per}(g)$ com período $\tau(p, g) \geq m$

$$
\prod_{i=0}^{k-1}\left\|D g^{m} \mid E^{s}\left(g^{m i}(p)\right)\right\| \leq K \lambda^{k}
$$

$e$

$$
\prod_{i=0}^{k-1}\left\|D g^{-m} \mid E^{u}\left(g^{-m i}(p)\right)\right\| \leq K \lambda^{k}
$$

onde $k=[\tau(p, g) / m]$. 
b) Para todo $0<i<\operatorname{dim} M$ deve existir uma decomposição contínua $T_{\Lambda_{i}(g)} M=E_{i} \oplus F_{i}$ tal que

$$
\left\|D g^{m}\left|E_{i}(x)\|\| D g^{-m}\right| F_{i}\left(g^{m}(x)\right)\right\| \leq \lambda,
$$

para todo $x \in \Lambda_{i}(g)$. E ainda, $E_{i}(p)=E_{p}^{s}(g), F_{i}(p)=E_{p}^{u}(g)$ quando $p \in \operatorname{Per}(g)$ e $\operatorname{dim} E_{p}^{s}(g)=i$.

c) Para todo $p \in \operatorname{Per}(g)$

$$
\limsup _{n \rightarrow+\infty} \frac{1}{n} \sum_{i=0}^{n-1} \log \left\|D g^{m} \mid E^{s}\left(g^{m i}(p)\right)\right\|<0
$$

$e$

$$
\limsup _{n \rightarrow+\infty} \frac{1}{n} \sum_{i=0}^{n-1} \log \left\|D g^{-m} \mid E^{u}\left(g^{-m i}(p)\right)\right\|<0
$$

Antes de mostrarmos esta proposição, vamos usá-la para demonstrar o Passo 1.

Fixemos agora $f \in \mathcal{G}_{m}^{1}(M)$. Desde que pela Proposição 3.1.1 os pontos periódicos hiperbólicos de $f$ possuem mesmo índice, digamos $s$, a Proposição 3.2.1 nos dá uma decomposição dominada $T_{\overline{\operatorname{Per}(f)}} M=E \oplus F$ para o conjunto $\overline{\operatorname{Per}(f)}$. Fixemos assim $m \in \mathbb{N}, 0<\lambda<1 \mathrm{e}$ $K>0$ como na Proposição 3.2.1.

Desta forma, para mostrarmos a hiperbolicidade de $\overline{\operatorname{Per}(f)}$ precisamos provar que de fato temos contração e expansão nos sub-fibrados $E$ e $F$, respectivamente, a menos de um certo número finito de iterados. O que devido a compacidade de $\overline{\operatorname{Per}(f)}$ torna suficiente mostrarmos o seguinte

$$
\liminf _{n \rightarrow+\infty}\left\|D f^{n} \mid E(x)\right\|=0
$$

$\mathrm{e}$

$$
\liminf _{n \rightarrow+\infty}\left\|D f^{-n} \mid F(x)\right\|=0
$$

para todo $x \in \overline{\operatorname{Per}(f)}$.

Notemos que é suficiente mostrarmos o primeiro caso, desde que o segundo pode ser deduzido do primeiro trocando $f$ por $f^{-1}$.

Desta forma, suponhamos que (3.4) não seja verdade. Então, existe $x \in \overline{\operatorname{Per}(f)}$ tal que

$$
\left\|D f^{j m} \mid E(x)\right\| \geq c>0, \text { para todo } j>0,
$$

onde $m$ é como na Proposição 3.2.1 fixado anteriormente. Definindo a seguinte medida de probabilidade

$$
\mu_{j}=\frac{1}{j} \sum_{i=0}^{j-1} \delta_{f^{m i}(x)},
$$

onde $\delta$ é a medida de Dirac, podemos encontrar uma subsequência $j_{n} \rightarrow \infty$ tal que $\mu_{j_{n}}$ convirja a uma medida de probabilidade $f^{m}$-invariante $\mu$ na topologia fraca* $\mathrm{e}$

$$
\lim _{n \rightarrow+\infty} \frac{1}{j_{n}} \log \left\|D f^{m j_{n}} \mid E(x)\right\| \geq \lim _{n \rightarrow+\infty} \frac{1}{j_{n}} \log c=0 .
$$


Sendo assim, tomando o funcional contínuo $\phi(y)=\log \left\|D f^{m} \mid E(y)\right\|$ sobre $\overline{\operatorname{Per}(f)}$, obtemos:

$$
\begin{aligned}
\int_{\overline{\operatorname{Per}(f)}} \phi d \mu & =\lim _{n \rightarrow+\infty} \frac{1}{j_{n}} \sum_{i=0}^{j_{n}-1} \log \left\|D f^{m} \mid E\left(f^{m i}(x)\right)\right\| \\
& \geq \lim _{n \rightarrow+\infty} \frac{1}{j_{n}} \log \left\|D f^{m j_{n}} \mid E(x)\right\| \geq 0 .
\end{aligned}
$$

E então, usando o Teorema Ergódico de Birkhoff

$$
0 \leq \int_{\overline{P e r(f)}} \phi d \mu=\int_{\overline{P e r(f)}} \lim _{n \rightarrow+\infty} \frac{1}{n} \sum_{i=0}^{n-1} \log \left\|D f^{m} \mid E\left(f^{m i}(y)\right)\right\| d \mu(y) .
$$

Seja $\Sigma(f) \subset M$ o conjunto de probabilidade total dado pelo ergodic closing lema. Sendo assim, denotando por $\nu=\frac{1}{m} \sum_{i=0}^{m-1}\left(f^{i}\right)^{*} \mu$ a medida de probabilidade $f$-invariante induzida por $\mu$, temos que $\nu(\Sigma(f) \cap \overline{\operatorname{Per}(f)})=1$ desde que $\nu$ é suportada sobre $\overline{\operatorname{Per}(f)}$. Notemos agora, que pela invariância de $\Sigma(f) \cap \overline{\operatorname{Per}(f)}$ por $f$ temos o seguinte,

$$
\begin{aligned}
0 & =\nu(\overline{\operatorname{Per}(f)}-\Sigma(f) \cap \overline{\operatorname{Per}(f)}) \\
& =\frac{1}{m} \sum_{i=0}^{m-1} \mu\left(f^{i}(\overline{\operatorname{Per}(f)}-\Sigma(f) \cap \overline{\operatorname{Per}(f)})\right) \\
& =\frac{1}{m} \sum_{i=0}^{m-1} \mu(\overline{\operatorname{Per}(f)}-\Sigma(f) \cap \overline{\operatorname{Per}(f)}) \\
& =\mu(\overline{\operatorname{Per}(f)}-\Sigma(f) \cap \overline{\operatorname{Per}(f)}),
\end{aligned}
$$

ou seja, $\Sigma(f)$ é um conjunto de medida total para $\mu$. E portanto, isto juntamente com (3.6) implica na existência de um ponto $y \in \Sigma(f) \cap \overline{\operatorname{Per}(f)}$ tal que:

$$
\lim _{n \rightarrow+\infty} \frac{1}{n} \sum_{i=0}^{n-1} \log \left\|D f^{m} \mid E\left(f^{m i}(y)\right)\right\| \geq 0 .
$$

Observemos que o item (c) da Proposição 3.2.1 é uma obstrução para que y seja periódico. Sendo assim, $y \notin \operatorname{Per}(f)$.

Por (3.7), podemos tomar $\lambda<\lambda_{0}<1$ e $n_{0}>0$ tal que:

$$
\frac{1}{n} \sum_{i=0}^{n-1} \log \left\|D f^{m} \mid E\left(f^{m i}(y)\right)\right\| \geq \log \lambda_{0}
$$

quando $n \geq n_{0}$.

A ideia agora é encontrar um ponto periódico hiperbólico $p \in \operatorname{Per}(g)$ cuja órbita esteja "próxima" da órbita de $y$, para $g$ suficientemente próximo de $f$, e assim usar o lema de Franks para trocar as derivadas na órbita de $p$, de tal maneira que a desigualdade (3.8) nos dê uma contradição com o item (a) da Proposição 3.2.1. 
Usando que $y \in \Sigma(f)$, podemos aproximar $f$ por um difeomorfismo $g$ tal que exista $p \in \operatorname{Per}(g)$ e a distância entre $g^{j}(p)$ e $f^{j}(y)$ seja arbitrariamente pequena, para $0 \leq j \leq n$, onde $n=\tau(p, g)$ é o período de $p$ para $g$. Desde que $y$ não é periódico o período $n$ vai para infinito quando $g$ aproxima-se de $f$.

Seja $U$ uma vizinhança de $\overline{\operatorname{Per}(f)}$ pequena o suficiente tal que o conjunto invariante maximal em $U$

$$
\Lambda_{U}(f)=\bigcap_{n \in \mathbb{Z}} f^{n}(U)
$$

possua uma decomposição dominada. Sendo assim, podemos tomar uma vizinhança $\mathcal{U} \subset$ $\mathcal{G}_{m}^{1}(M)$ de $f$ nas condições da Proposição 3.2 .1 e ainda tal que todo $\Lambda_{U}(h)$ possua uma decomposição dominada variando continuamente com $\Lambda_{U}(f)$ se $h \in \mathcal{U}$. Denotamos esta decomposição por $T_{\Lambda_{U}(h)} M=E_{h} \oplus F_{h}$. Notemos que pela Proposição 3.2.1 temos $E_{g}(p)=$ $E_{g}^{s}(p)$ e $F_{g}(p)=E_{g}^{u}(p)$, e pela continuidade da decomposição podemos supor $E_{g}^{s}\left(g^{i}(p)\right)$ e $E_{g}^{u}\left(g^{i}(p)\right)$ tão próximo quanto se queira de $E_{f}\left(f^{i}(y)\right)$ e $F_{f}\left(f^{i}(y)\right), 0 \leq i \leq n$, respectivamente, diminuindo a vizinhança $\mathcal{U}$, se necessário.

Na sequência, construímos um isomorfismo conservativo $A_{i}: T_{g^{i}(p)} M \rightarrow T_{f^{i}(y)} M, 0 \leq i \leq$ $n$, próximo da identidade em coordenadas locais. Mais ainda, estes isomorfismos são tais que $A_{i}\left(E_{g}^{s}\left(g^{i}(p)\right)\right)=E_{f}\left(f^{i}(y)\right)$ seja uma isometria.

Mostremos agora como construir $A_{0}$, sendo completamente análogo os outros casos. Para tal, fixemos

$$
\left\{e_{1}(l), \ldots, e_{s}(l), r_{1}(l), \ldots, r_{d-s}(l)\right\} \text { uma base ortonormal de } T_{l} M, l=y, p,
$$

tal que $\left\{e_{j}(l), 1 \leq j \leq s\right\}$ seja uma base ortonormal para $E_{f}(y)$ se $l=y$ ou para $E_{g}^{s}(p)$ se $l=p$. Observe que pela continuidade da decomposição dominada estas bases podem ser tomadas próximas, de tal forma que a aplicação $A_{0}: T_{p} M \rightarrow T_{y} M$ satisfazendo $A_{0}\left(e_{l}(p)\right)=e_{l}(y)$ e $A_{0}\left(r_{j}(p)\right)=r_{j}(y), 1 \leq l \leq s$ e $s+1 \leq j \leq d$, seja próxima da identidade. Notemos também, que por construção $A_{0}$ é uma aplicação conservativa e uma isometria restrita ao espaço $E_{g}^{s}(p)$ como queríamos.

Agora, voltemos a demonstração. Seja $L_{i}: T_{g^{i}(p)} M \rightarrow T_{g^{i+1}(p)} M$ uma aplicação conservativa definida como segue

$$
L_{i}=A_{i+1}^{-1} D f_{f^{i}(y)} A_{i}, \text { para } 0 \leq i \leq n-1 .
$$

Tomando $n$ maior (i.e., $g$ mais próxima de $f$ ), se necessário, $L_{i}$ é tão próximo de $D g_{g^{i}}(p)$ quanto se queira, para todo $0 \leq i \leq n-1$. Então, usando o lema de Franks, podemos encontrar $h \in \mathcal{U}$ tal que $p \in \operatorname{Per}(h)$ e $D h_{h^{i}(p)}=L_{i}, 0 \leq i \leq n-1$. Observemos que, por construção das $L_{i}^{\prime} \mathrm{s}, E_{g}^{s}(p) \subset T_{p} M$ ainda é invariante por $D h_{p}^{n}$, e mais, pela continuidade da decomposição dominada em $\mathcal{U}$ podemos assumir que $E_{p}^{s}(g) \cap F_{h}(p)=\{0\}$. Sendo assim, $E_{p}^{s}(g)=E_{h}(p)$ e desde que $h \in \mathcal{U}$, pela Proposição 3.2.1 temos que de fato $E_{h}^{s}(p)=E_{g}^{s}(p)$.

Finalmente, como $A_{i} \mid E_{g}^{s}\left(g^{i}(p)\right)$ é uma isometria, temos o seguinte

$$
\left\|D h^{m}\left|E_{h}^{s}\left(h^{i m}(p)\right)\|=\| D f^{m}\right| E\left(f^{i m}(y)\right)\right\| \text {, para todo } i \in \mathbb{N} .
$$


Portanto, para $k=[n / m]$ temos

$$
\prod_{i=0}^{k-1}\left\|D h^{m}\left|E_{h}^{s}\left(h^{i m}(p)\right)\left\|=\prod_{i=0}^{k-1}\right\| D f^{m}\right| E\left(f^{i m}(y)\right)\right\| \geq \lambda_{0}^{k},
$$

o que contradiz a Proposição 3.2.1, mostrando assim que de fato $\overline{\operatorname{Per}(f)}$ é um conjunto hiperbólico se $f \in \mathcal{G}_{m}^{1}(M)$.

\section{Demonstração Passo 2:}

Para mostrarmos que $\Omega(f)=\overline{\operatorname{Per}(f)}$ usamos o closing lema.

Se $f \in \mathcal{G}_{m}^{1}(M)$, então existe uma vizinhança conexa $\mathcal{U}$ de $f$ em $\operatorname{Diff}_{m}^{1}(M)$ onde $\# H_{n}(g)$, o número de pontos periódicos hiperbólicos de $g$ com período menor ou igual do que $n$, é finito e constante para todo $g \in \mathcal{U}$, desde que todos os difeomorfismos em $\mathcal{U}$ possuem apenas pontos periódicos hiperbólicos.

Suponhamos agora que $\overline{\operatorname{Per}(f)} \subsetneq \Omega(f)$, e seja $x \in \Omega(f) \backslash \overline{\operatorname{Per}(f)}$. Pelo closing lema, podemos fixar $k \in \mathbb{N}$ tal que para criarmos um ponto periódico hiperbólico $p$ de um difeomorfismo $g \in \mathcal{U}$ próximo do ponto $x$, as perturbações são feitas em uma vizinhança arbitrariamente pequena de

$$
\bigcup_{-k \leq j \leq k} f^{j}(x)
$$

Mais precisamente, $f=g$ no complementar desta vizinhança. Então, seja $U$ uma vizinhança de $\overline{\operatorname{Per}(f)}$ tal que $f^{j}(x) \notin \bar{U},-k \leq j \leq k$. Usando então o closing lema podemos encontrar $g \in \mathcal{U}$ e $p \in \operatorname{Per}(g) \cap U^{c}$. Logo, $p \in H_{n}(g)$ para algum $n>0$. Entretanto, pela escolha da vizinhança $U, f(y)=g(y)$ para $y \in U$, e portanto $\# H_{n}(f) \neq \# H_{n}(g)$ desde que $p \notin \bar{U}$. O que nos leva a uma contradição com a escolha da vizinhança $\mathcal{U}$ de $f$.

Portanto, temos que $\Omega(f)=\overline{\operatorname{Per}(f)}$ o que completa a prova do passo 2 .

Vamos agora mostrar a Proposição 3.2.1. Na verdade, esta é uma consequência do lema de Franks e um notável resultado de algebra linear apresentado por Mañé em [35].

Primeiro recordemos algumas noções. Seja $G L(d)$ o grupo linear de isomorfismos. Se $\xi: \mathbb{Z} \rightarrow G L(d)$ é uma sequência de isomorfismos em $\mathbb{R}^{d}$, denotemos por $E_{i}^{s}(\xi)$ (resp. $E_{i}^{u}(\xi)$ ) o espaço de vetores $v \in \mathbb{R}^{d}$ tal que

$$
\sup \left\{\left\|\left(\prod_{j=0}^{n} \xi_{i+j}\right) v\right\| ; n \geq 0\right\}<\infty,\left(\operatorname{resp} . \sup \left\{\left\|\left(\prod_{j=0}^{n}\left(\xi_{i-1-j}\right)^{-1}\right) v\right\| ; n \geq 0\right\}<\infty\right) \text {. }
$$

Dizemos que a sequência é hiperbólica se $E_{i}^{s}(\xi) \oplus E_{i}^{u}(\xi)=\mathbb{R}^{d}$ para todo $i \in \mathbb{Z}$. A sequência é periódica se existe $m$ tal que $\xi_{i+m}=\xi_{i}$ para $i \in \mathbb{Z}$, e o menor inteiro positivo $m$ que satisfaça isto é chamado o período da sequência. E então, a hiperbolicidade da sequência neste caso é equivalente a matriz $\prod_{i=0}^{m-1} \xi_{i}$ ser hiperbólica.

Seja $\left\{\xi^{(\alpha)}, \quad \alpha \in \mathcal{A}\right\}$ uma família de sequências periódicas de aplicações lineares. Dizemos que esta família é hiperbólica se toda sequência da família é hiperbólica e $\sup \left\{\left\|\xi_{n}^{\alpha}\right\|, \alpha \in\right.$ 
$\mathcal{A}, n \in \mathbb{Z}\}<\infty$. Dado duas famílias de sequências periódicas $\xi$ e $\eta$, definimos a distância entre elas como

$$
d(\xi, \eta)=\sup _{\alpha, i}\left\{\left\|\xi_{i}^{(\alpha)}-\eta_{i}^{(\alpha)}\right\|\right\} .
$$

Dizemos que elas são equivalentes se para todo $\alpha$ o período de $\xi^{\alpha}$ e $\eta^{\alpha}$ coincidem. Finalmente dizemos que a família $\xi$ é uniformemente hiperbólica se existe $\varepsilon>0$ tal que para toda família equivalente $\eta \varepsilon$-próxima de $\xi, d(\xi, \eta)<\varepsilon$, é também hiperbólica.

A prova da Proposição 3.2.1 seguirá da proposição seguinte para sequências periódicas.

Proposição 3.2.2 (Lema II.3 em [35]). Se $\left\{\xi^{\alpha}\right\}$ é uma família hiperbólica uniforme, então existem constantes $K>0, m \in \mathbb{N}$ e $0<\lambda<1$ tal que

1. Se $\xi^{\alpha}$ tem periodo $n \geq m$ e $k=[n / m]$ então

$$
\prod_{j=0}^{k-1}\left\|\left(\prod_{i=0}^{m-1} \xi_{m j+i}^{\alpha}\right)\left|E_{m j}^{s}\left\|\leq K \lambda^{k} e \prod_{j=0}^{k-1}\right\|\left(\prod_{i=0}^{m-1} \xi_{m j+i}^{\alpha}\right)^{-1}\right| E_{m(j+1)}^{u}\right\| \leq K \lambda^{k}
$$

2. Para todo $\alpha$ e $j \in \mathbb{Z}$ :

$$
\left\|\left(\prod_{i=0}^{m-1} \xi_{j+i}^{\alpha}\right)\left|E_{j}^{s}\|\|\left(\prod_{i=0}^{m-1} \xi_{j+i}^{\alpha}\right)^{-1}\right| E_{j+m}^{u}\right\| \leq \lambda .
$$

3. Para todo $\alpha$ :

$$
\begin{gathered}
\limsup _{n \rightarrow \infty} \frac{1}{n} \sum_{j=0}^{n-1} \log \left\|\left(\prod_{i=0}^{m-1} \xi_{m j+i}^{\alpha}\right) \mid E_{m j}^{s}\right\|<0 e \\
\limsup _{n \rightarrow \infty} \frac{1}{n} \sum_{j=0}^{n-1} \log \left\|\left(\prod_{i=0}^{m-1} \xi_{m j+i}^{\alpha}\right)^{-1} \mid E_{m(j+1)}^{u}\right\|<0 .
\end{gathered}
$$

Demonstração da Proposição 3.2.1:

Seja $f \in \mathcal{G}_{m}^{1}(M)$. Agora, seja $\mathcal{U}$ vizinhança de $f$ em $\mathcal{G}_{m}^{1}(M)$ e escolha $\mathcal{U}_{0} \subset \mathcal{U}$ e $\varepsilon>0$ vizinhança de $f$ e uma constante positiva, respectivamente, dados pelo lema de Franks.

Afirmação: A familia de sequências periódicas $\left\{\xi^{(x, g)} ; g \in \mathcal{U}_{0}, x \in \operatorname{Per}(g)\right\}$, onde $\xi_{j}^{(x, g)}=$ $D g_{g^{j}(x)}$, é uniformemente hiperbólica.

Suponhamos que isto não seja verdade. Logo, existe uma família $\left\{\eta^{(x, g)} ; g \in \mathcal{U}_{0}, x \in\right.$ $\operatorname{Per}(g)\}$ tal que $d(\xi, \eta) \leq \varepsilon$, para algum $g \in \mathcal{U}_{0}, x \in \operatorname{Per}(g)$, as sequências $\eta^{(x, g)}$ e $\xi^{(x, g)}$ possuem o mesmo período e $\eta^{(x, g)}$ não é hiperbólica. Notemos que apesar desta sequência periódica não ser necessariamente formada por matrizes conservativas, multiplicando cada matriz pelo inverso do seu determinante numa direção distinta da não hiperbólica podemos assumir que a sequência é formada por matrizes conservativas, desde que os determinantes das matrizes $\eta^{(x, g)}$ eram próximos de um. 
Agora, usando o lema de Franks podemos encontrar $h \in \mathcal{U}$ tal que $h\left(g^{i}(x)\right)=g^{i+1}(x)$ para todo $i$ e $D h_{g^{i}(x)}=\eta_{i}^{(x, g)}$. Sendo assim, temos um ponto periódico não hiperbólico $x$ de $h$ em $\mathcal{U} \subset \mathcal{G}_{m}^{1}(M)$ o que gera uma contradição, e portanto mostra ser verdade a afirmação.

Pela a afirmação, podemos usar a Proposição 3.2 .2 para a família $\left\{\xi^{(x, g)} ; g \in \mathcal{U}_{0}, x \in\right.$ $\operatorname{Per}(g)\}$. Logo, as propriedades $(a)$ e (c) seguem direto dos item (1) e (3) da Proposição 3.2.2, respectivamente. Agora, o item (2) da Proposição 3.2.2 nos diz que a decomposição por variedades estáveis e instáveis em $\operatorname{Per}(g), g \in \mathcal{U}_{0}$, é contínua e dominada, logo esta se estende para o fecho e assim temos a propriedade $(b)$.

\subsection{Conjectura de Palis no mundo conservativo}

Como já foi dito na introdução, precisamos apenas provar a conjectura de Palis para o caso em que a dimensão de $M$ é maior do que dois, $d>2$, desde que em dimensão dois difeomorfismos conservativos são simpléticos, para os quais temos uma prova da conjectura dada por Newhouse [38]. Veja Apêndice B.

Lembremos agora que na demonstração da Proposição 3.1.1 mostramos que um difeomorfismo conservativo possuindo dois pontos periódicos hiperbólicos com índices diferentes pode ser perturbado de tal maneira a exibir um ciclo heterodimensional. Sendo assim, para concluirmos a prova da Conjectura no caso conservativo precisamos apenas mostrar que difeomorfismos no complementar dos Anosov, a menos de uma perturbação, possuem pontos periódicos hiperbólicos com índices diferentes. Para tal, usamos o Teorema E.

Primeiro, lembremos como perturbar um difeomorfismo a fim de transformar pontos periódicos não hiperbólicos em hiperbólicos.

Mais geral, seja $f \in \operatorname{Diff}_{m}^{1}(M)$ e $p$ um ponto periódico de $f$ com período $\tau=\tau(p, f)$ tal que

$$
D f_{p}^{\tau}=\left[\begin{array}{ccccc}
a_{1} & b_{1} & & & \\
-b_{1} & a_{1} & * & & \\
& & \ddots & * & \\
& & & a_{t} & b_{t} \\
0 & & & -b_{t} & a_{t}
\end{array}\right]
$$

para alguma base de $T_{p} M$, e algum $t \geq d / 2$ desde que alguns blocos na diagonal podem ser $1 \times 1$. Sendo assim, os autovalores são $a_{j} \pm i b_{j}$. Agora, fixados dois blocos distintos $j$ e $i$, usando o lema de Franks para $c$ próximo de um, podemos construir uma perturbação da identidade $h$ tal que $h=I d$ no complementar de alguma vizinhança pequena $U$ de $p, h(p)=p$ e $D h(p)=\left(a_{k l}\right)$, onde $a_{j j}=c, a_{i i}=1 / c, a_{k k}=1$ para $k \neq i, j$ e $a_{k l}=0$ para todo $k \neq l$. Então, considerando $g=h \circ f$ temos que $g$ é próximo de $f, p$ ainda é um ponto periódico de 
$g$ e

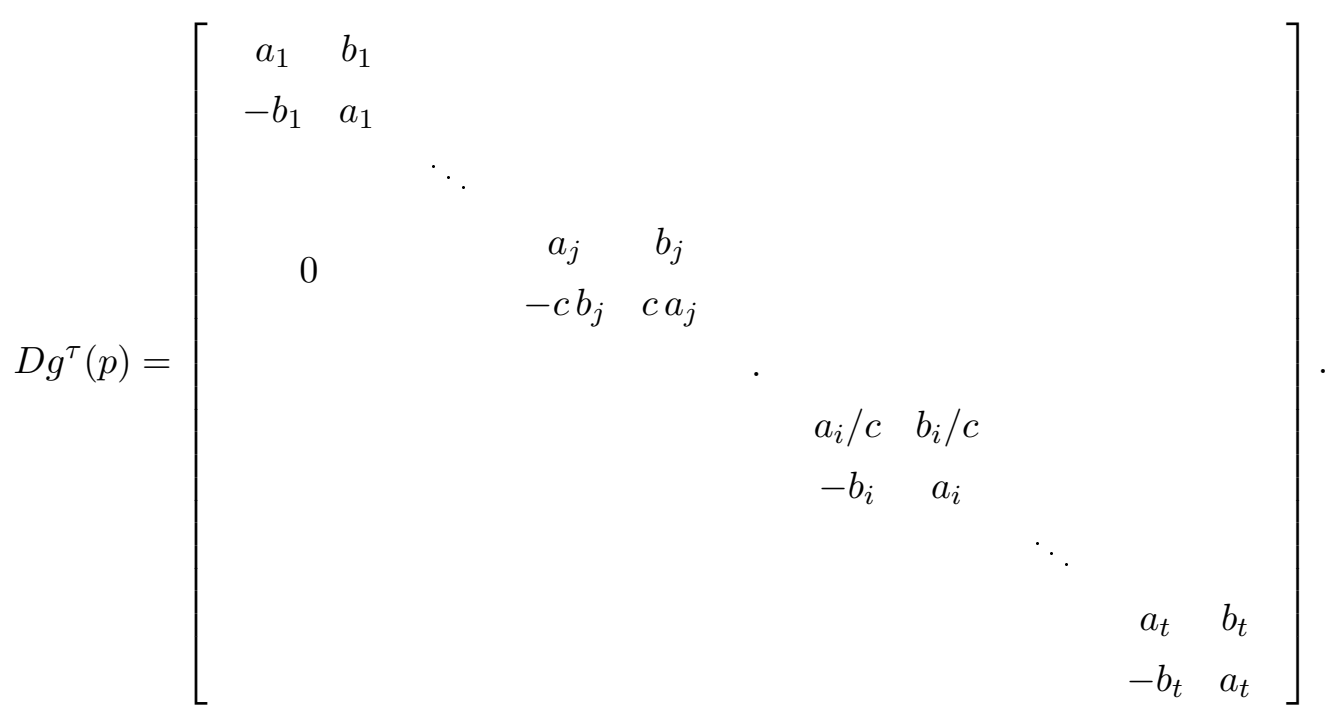

Agora, é fácil checar que o módulo ao quadrado dos autovalores de $D g^{\tau}$ são

$$
a_{1}^{2}+b_{1}^{2}, \ldots, c\left(a_{j}^{2}+b_{j}^{2}\right), \ldots, 1 / c\left(a_{i}+b_{i}\right), \ldots .
$$

Desta forma, podemos construir perturbações $h$ da $i d$ a fim de transformar pontos periódicos não hiperbólicos em hiperbólicos.

Voltemos então a demonstração da conjectura. Seja $f \in \operatorname{Diff}_{m}^{1}(M)$ um difeomorfismo não Anosov. Então, pelo Teorema $\mathrm{E}$ temos que $f \notin \mathcal{G}_{m}^{1}(M)$, e portanto depois de uma perturbação podemos supor que $f$ possui um ponto periódico $p_{1}$ não hiperbólico. Sem perda de generalidade, podemos também supor que exista um ponto periódico hiperbólico $p_{2}$ para $f$. Agora, se $d=\operatorname{dim} M \neq 4$ podemos usar uma perturbação local numa vizinhança de $p_{1}$ como apresentado acima, escolhendo $c>0$ e blocos apropriados tal que $p_{1}$ e $p_{2}$ sejam pontos periódicos hiperbólicos de $f$ com índices diferentes, como queríamos.

O problema para $d=4$ é que o ponto periódico $p_{2}$ poderia ter índice dois, e o ponto periódico não hiperbólico $p_{1}$ ser tal que $D f_{p_{1}}^{\tau\left(p_{1}, f\right)}$ possua apenas autovalores complexos de módulo um. Desta forma, a perturbação local acima tornaria sempre $p_{1}$ periódico hiperbólico com índice dois. No entanto, usando a perturbação acima, o pasting lema e o Lema 1.2.1 podemos encontrar um ponto periódico hiperbólico $\tilde{p}_{1}$ próximo de $p_{1}$ com período $n \tau\left(p_{1}, f\right)$ tal que $D f_{\tilde{p}_{1}}^{n \tau\left(p_{1}, f\right)}$ possua pelo menos dois autovalores reais. Desde que estes autovalores são tão próximos de um quanto se queria, podemos fazer uma perturbação local como acima (neste caso temos blocos $1 \times 1$ ) e agora sim obter um ponto periódico hiperbólico $\tilde{p}_{1}$ com índice diferente de $p_{2}$. 
Neste capítulo vamos demonstrar em linhas gerais a Proposição 2.1.3 seguindo a demonstração deste resultado no caso dissipativo dada por Abdenur, Bonatti e Crovisier em [1].

Lembremos primeiramente o enunciado.

Proposição 2.1.3. Existe um subconjunto residual $\mathcal{R} \subset \operatorname{Diff}_{m}^{1}(M)$ tal que se $f \in \mathcal{R}$ e $\mu$ é uma medida ergódica para $f$, então existem medidas periódicas $\mu_{p}$ convergindo para $\mu$ na topologia fraca*, e mais ainda os vetores formados pelos expoentes de Lyapunov de $\mu_{p}$, $L\left(\mu_{p}\right) \in \mathbb{R}^{d}$, também convergem ao vetor de Lyapunov $L(\mu) \in \mathbb{R}^{d}$.

\section{A.1 Demonstração da Proposição 2.1.3}

A demonstração, a menos de argumentos genéricos como podemos ver abaixo, é direto da proposição seguinte.

Proposição A.1.1. Seja $\mu$ uma medida de probabilidade ergódica invariante para um difeomorfismo conservativo $f$ de uma variedade compacta $M$. Seja $\mathcal{U} \subset \operatorname{Diff}_{m}^{1}(M)$ uma $C^{1}$ vizinhança de $f, \mathcal{V}$ uma vizinhança de $\mu$ no espaço das probabilidades munido da topologia fraca*, $\mathcal{K}$ uma Hausdorff vizinhança do suporte de $\mu$, e $\mathcal{O}$ uma vizinhança do vetor de Lyapunov $L(\mu) \in \mathbb{R}^{d}$. Então, existe $g \in \mathcal{U}$ e uma órbita periódica $\gamma$ de $g$ tal que a medida Dirac $\mu_{\gamma}$ associada a $\gamma$ pertence a $\mathcal{V}$, seu suporte está em $\mathcal{K}$, e seu vetor de Lyapunov $L\left(\mu_{\gamma}\right)$ pertence $a \mathcal{O}$. 
Usemos então a Proposição A.1.1 para provarmos a Proposição 2.1.3.

Notemos primeiro que como o espaço dos difeomorfismos $C^{1}$ sobre $M$ é um conjunto separável é suficiente mostrarmos o resultado restrito a uma pequena vizinhança $\mathcal{U}$ de um difeomorfismo arbitrário $f_{0} \in \operatorname{Diff}_{m}^{1}(M)$. Em particular, temos que $\log \|D f\|$ e $\log \left\|D f^{-1}\right\|$ são limitados por alguma constante $S>0$, para todo $f \in \mathcal{U}$.

Consideremos agora $X$ o espaço das triplas $(\mu, K, L)$ onde $\mu$ é uma medida de probabilidade em $M, K \subset M$ é um subconjunto compacto, e $L \in[-S, S]^{d}$, munido com a topologia produto da topologia fraca* sobre as medidas de probabilidades, a topologia de Hausdorff sobre os subconjuntos compactos de $M$, e a topologia usual de $\mathbb{R}^{d}$.

Para uma órbita periódica $\gamma$ de um difeomorfismo $f$ associamos a tripla $x_{\gamma}=\left(\mu_{\gamma}, \gamma, L\left(\mu_{\gamma}\right)\right)$, e denotamos por $X_{f}$ o fecho do conjunto $\left\{x_{\gamma}, \gamma \in \operatorname{Per}(f)\right\}$. Note que $X_{f}$ é um subconjunto compacto de $X$ e assim é um elemento do espaço $\mathcal{K}(X)$ formado pelos subconjuntos compactos de $X$ munidos com a topologia de Hausdorff.

Pela continuidade das órbitas periódicas hiperbólicas (e elípticas no caso conservativo bidimensional) temos que a aplicação $f \mapsto X_{f}$ definida no subconjunto residual dos difeomorfismos Kupka-Smale, veja [44], subconjunto este formado pelos difeomorfismos que possuem apenas pontos periódicos hiperbólicos (e/ou elípticos no caso bidimensional), é semicontínua inferior. Como consequência, esta aplicação é contínua num subconjunto residual $\mathcal{R} \cap \mathcal{U}$ do conjunto dos difeomorfismos Kupka-Smale, e portanto de $\mathcal{U}$.

Consideremos $f \in \mathcal{R} \cap \mathcal{U}$ e $\mu$ uma medida de probabilidade ergódica para $f$. A Proposição A.1.1 nos permite encontrar uma órbita periódica $\gamma$ de um difeomorfismo $g$ próximo de $f$ tal que $x_{\gamma}$ é arbitrariamente próximo de $(\mu, \operatorname{supp}(\mu), L(\mu))$; e a menos de uma perturbação, podemos assumir $\gamma$ ser hiperbólica (e/ou elíptica para superfícies), e $g$ ser um difeomorfismo Kupka-Smale. Agora, desde que $f$ é um ponto de continuidade da aplicação $g \mapsto X_{g}$ no conjunto dos difeomorfismos Kupka-Smale, temos que $(\mu, \operatorname{supp}(\mu), L(\mu))$ pertence a $X_{f}$, o que implica a Proposição 2.1.3.

\section{A.2 Demonstração da Proposição A.1.1}

Fixemos uma medida ergódica para um difeomorfismo conservativo $f$ sobre $M$. Seja $\lambda_{1}<\ldots<\lambda_{k}$ os expoentes de Lyapunov de $\mu$ e para cada $i$ seja $d_{i}$ a multiplicidade dos expoentes $\lambda_{i}$.

Agora, pelo Teorema Ergódico de Birkhoff e o Teorema subaditivo de Oseledets temos um conjunto de medida total $\Gamma$ para $\mu$ tal que se $x \in \Gamma$ então:

- As medidas de probabilidade $\frac{1}{n} \sum_{0}^{n-1} \delta_{f^{i}(x)}$ e $\frac{1}{n} \sum_{0}^{n-1} \delta_{f^{-i}(x)}$ convergem a $\mu$ quando $n \rightarrow+\infty$;

- existe uma decomposição $T_{x} M=E_{1} \oplus \ldots \oplus E_{k}$ tal que:

$$
-\operatorname{dim}\left(E_{i}\right)=d_{i}
$$


- o número $\frac{1}{n} \log \left\|D f^{n}(u)\right\|$ converge uniformemente para $\lambda_{i}$, para os vetores unitários $u$ em $E_{i}$, quando $n \rightarrow \infty$;

- o ângulo entre os espaços $D f^{n}\left(E_{i}\right)$ e $D f^{n}\left(E_{j}\right)$ decrescem no máximo subexponencialmente:

$$
\lim _{n \rightarrow+\infty} \frac{1}{n} \log \operatorname{sen}\left(\angle\left(E_{i}, E_{j}\right)\right)=0
$$

Consideremos $\Sigma=\Gamma \cap \Sigma(f)$, onde $\Sigma(f)$ é o conjunto de medida total dado pelo ergodic closing lema. Sendo assim, $\Sigma$ tem medida total para $\mu$, em particular existe um ponto $x \in \Sigma$. Fixemos uma coordenada local em $x$ tal que

$$
E_{i}=\{0\}^{\sum_{1}^{i-1} d_{l}} \times \mathbb{R}^{d_{i}} \times\{0\}^{\sum_{i+1}^{k} d_{l}}
$$

e fixemos também uma métrica Riemanianna sobre $M$ que coincida com a métrica Euclidiana nesta coordenada. Para $i \leq j$ denotemos por $E_{i, j}=E_{i} \oplus \ldots \oplus E_{j}$.

Dividimos a demonstração da Proposição A.1.1 em dois lemas. No primeiro construímos a perturbação, e no segundo verificamos ser verdade as propriedades anunciadas para tal.

Lema A.2.1. Para toda vizinhança $\mathcal{U}$ de $f$, e $\varepsilon>0$ existem:

- um número $C>0$,

- uma sequência $\left(\varepsilon_{n}\right)$ de números positivos com $\lim _{n \rightarrow \infty} \varepsilon_{n}=0$,

- uma sequência de inteiros $t_{n} \rightarrow+\infty$,

- uma sequência de difeomorfismos $f_{n} \in \mathcal{U}$,

com as seguintes propriedades:

a) existe um ponto periódico $x_{n}$ de $f_{n}$ com período $t_{n}$, tal que $d\left(f^{i}(x), f_{n}^{i}\left(x_{n}\right)\right)<\varepsilon_{n}$ para $i \in\left\{0, \ldots, t_{n}\right\}$.

b) A expressão de $D f_{n}^{t_{n}}(x)$ em coordenadas locais no ponto $x$ coincide com $P_{n} \circ D f^{t_{n}}(x)$.

c) Para todo $i \leq j \in\{1, \ldots, k\}$ a inclinação de $D f_{n}^{t_{n}}\left(x_{n}\right)\left(E_{i, j}\right)$ com respeito a $E_{i, j}$ é menor do que $C$.

Como $x \in \Sigma$, as medidas Dirac definidas ao longo das órbitas periódicas de $x_{n}$ para $f_{n}$ convergem fracamente para $\mu$, e ainda estas órbitas convergem para o suporte de $\mu$ na topologia Hausdorff, quando $n \rightarrow \infty$. Então, concluímos a demonstração da Proposição A.1.1 provando:

Lema A.2.2. Os vetores de Lyapunov das órbitas de $x_{n}$ para $f_{n}$ convergem ao vetor de Lyapunov de $\mu$ para $f$, quando $n \rightarrow \infty$. 
Demonstração do Lema A.2.1:

Primeiro escolhemos uma sequência qualquer $\varepsilon_{n}$ convergindo para zero. Feito isto, usamos o ergodic closing lema para encontrarmos difeomorfismos $f_{n}$ em $\mathcal{U}$ e pontos periódicos $x_{n} \in \operatorname{Per}\left(f_{n}\right)$, tal que $d\left(f^{i}(x), f_{n}^{i}\left(x_{n}\right)\right)<\varepsilon_{n}$, para $0 \leq i \leq t_{n}$, onde $t_{n}$ é o período de $x_{n}$. Como $x$ é um ponto não periódico é direto que $t_{n} \rightarrow \infty$ quando $n \rightarrow \infty$. Agora, análogo ao feito na demonstração do passo 1 do Teorema E, usando o lema de Franks podemos assumir que $D f_{n}\left(f_{n}^{i}\left(x_{n}\right)\right)=D f\left(f^{i}(x)\right)$ (em coordenadas locais), para todo $0 \leq i \leq t_{n}-1$, e portanto $D f_{n}^{t_{n}}\left(x_{n}\right)=D f^{t_{n}}(x)$.

Continuando, podemos escolher $C>0$ grande o suficiente dependendo de $\eta$ e $\|f\|$ tal que exista uma matriz ortogonal $P_{n} \in O(\mathbb{R}, d) \varepsilon$-próxima da aplicação identidade tal que $P_{n} \circ D f^{t_{n}}(x)\left(E_{i, j}\right)$ não pertença a $1 / C$-vizinhança de $E_{i, j}^{\perp}$, ou seja, o ângulo $\angle\left(E_{i, j}, P_{n}\right.$ ○ $\left.D f^{t_{n}}(x)\left(E_{i, j}\right)\right)<C$, para todo $i<j$. E assim, usando uma vez mais o lema de Franks, podemos supor que a perturbação $f_{n}$ de $f$ acima é tal que $D f_{n}^{t_{n}}\left(x_{n}\right)=P_{n} \circ D f^{t_{n}}(x)$ e logo concluímos o lema.

Demonstração do Lema A.2.2: Consideremos os espaços de Lyapunov $E_{1}, \ldots, E_{k}$ de $x$ e para todo $j \in\{1, \ldots, k-1\}$ denotemos $F_{j}=E_{1} \oplus \ldots \oplus E_{j}=E_{1, j}$ e $G_{j}=E_{j+1} \oplus$ $\ldots \oplus E_{k}=E_{j+1, k}$. Denotemos por $m(A)$ a conorma da aplicação linear $A \in G L(\mathbb{R}, d)$, i.e., $m(A)=\left\|A^{-1}\right\|^{-1}$. Pelo Teorema de Oseledets temos o seguinte lema.

Lema A.2.3. Para todo $\nu>0$, existe $n_{\nu} \geq 1$ tal que para todo $n \geq n_{\nu}$ e $j \in\{1, \ldots, k-1\}$ temos:

$$
\begin{aligned}
& \frac{1}{n} \log \left(\left\|D f^{n} \mid F_{j}\right\|\right) \leq \lambda_{j}+\frac{1}{2} \nu \quad e \quad \frac{1}{n} \log \left(m\left(D f^{n} \mid G_{j}\right)\right) \geq \lambda_{j+1}-\nu, \\
& \frac{1}{n} \log \left(m\left(D f^{-n} \mid F_{j}\right)\right) \geq-\lambda_{j}-\frac{1}{2} \nu \quad e \quad \frac{1}{n} \log \left(\left\|D f^{-n} \mid G_{j}\right\|\right) \geq-\lambda_{j+1}+\nu .
\end{aligned}
$$

Para $K>0$, seja $C_{j, K}^{u}$ o cone de vetores cuja inclinação com respeito a $G_{j}$ é menor do que $K$ :

$$
C_{j, K}^{u}=\left\{v=v^{s}+v^{u} \in T_{x} M: v^{s} \in F_{j}, v^{u} \in G_{j},\left\|v^{s}\right\| \leq K\left\|v^{u}\right\|\right\} .
$$

Denotemos agora por $C_{j, K}^{s}$ o fecho de $T_{x} M \backslash C_{j, K}^{u}$. Agora, usando o Lema A.2.3 e a existência de $C>0$ para $f_{n}$ como no Lema A.2.1 podemos mostrar que os cones $C_{j, 4 C}^{u}$ e $C_{j, 4 C}^{s}$ são invariantes para $D f_{n}^{t_{n}}$ e $D f_{n}^{-t_{n}}$, respectivamente. Mais ainda, para $\nu>0$ arbitrariamente pequeno, temos que

$$
\frac{1}{t_{n}} \log \left(\left\|D f_{n}^{t_{n}}(v)\right\|\right) \leq \lambda_{j+1}-\nu, \text { para todo vetor unitário } v \in C_{j, K}^{u},
$$

e

$$
\frac{-1}{t_{n}} \log \left(\left\|D f_{n}^{-t_{n}}(w)\right\|\right) \leq \lambda_{j}+\nu, \text { para todo vetor unitário } w \in C_{j, K}^{s} .
$$

E assim, $D f_{n}^{t_{n}}\left(x_{n}\right)$ admitem espaços vetorias invariantes (únicos) $G_{i}^{n}$ e $F_{i}^{n}$ com dimensões $\operatorname{dim}\left(G_{i}\right)$ e $\operatorname{dim}\left(F_{i}\right)$, pertencendo aos cones $C_{i, 4 C}^{u}$ e $C_{i, 4 C}^{s}$, respectivamente. Mais ainda, estes 
são tais que $m\left(D f_{n}^{t_{n}}\left(x_{n}\right) \mid G_{i}^{n}\right)>\lambda_{i+1}-\nu$ e $\left\|D f_{n}^{t_{n}}\left(x_{n}\right) \mid F_{i}^{n}\right\|>\lambda_{i}+\nu$. Portanto, tomando $E_{i}^{n}=F_{i}^{n} \cap G_{i}^{n}$ temos que $\operatorname{dim}\left(E_{i}^{n}\right)=\operatorname{dim}\left(E_{i}\right)$ e os expoentes de Lyapunov de $x_{n}$ na direção $E_{i}^{n}$ estão contidos no intervalo $\left[\lambda_{i}-\nu, \lambda_{i}+\nu\right]$. O que prova que para $n \rightarrow \infty$ o vetor de Lyapunov de $x_{n}$ para $f_{n}$ converge ao vetor de Lyapunov de $\mu$ como queríamos. 



\section{Passo 1 da Proposição 2.2.1}

Antes de mais nada relembremos o que queremos demonstrar.

Passo 1 da Proposição 2.2.1: Seja $f \in \mathcal{H} \subset \operatorname{Diff}_{\omega}^{1}(M)$ um difeomorfismo não Anosov e $p$ um ponto periódico hiperbólico de $f$, então existe $g_{1} C^{1}$-próximo de $f$ tal que $p$ ainda seja um ponto periódico hiperbólico de $g_{1}$, e $g_{1}$ exiba uma tangência homoclínica entre $W^{s}\left(o(p), g_{1}\right)$ e $W^{u}\left(o(p), g_{1}\right)$. Mais ainda, $g_{1}=D f$ numa vizinhança pequena da órbita de $p$ (em coordenadas locais).

Desde que $\mathcal{H}$ é um subconjunto residual em $\operatorname{Diff}_{\omega}^{1}(M)$ e este é um espaço de Baire, claramente o passo acima é uma prova da conjectura de Palis no caso simplético, i.e., os difeomorfismos simpléticos ou são aproximados por difeomorfismos hiperbólicos ou por difeomorfismos exibindo tangência homoclínica, lembre-se que no caso simplético os índices dos pontos periódicos hiperbólicos são constantes e portanto não temos a presença de ciclos heterodimensionais. Na verdade a prova da conjectura de Palis (construção da tangência homoclínica) aparece na demonstração do Teorema 1.1 em [38] (passo 6), no qual Newhouse prova que o conjunto $\mathcal{G}_{\omega}^{1}(M)$, conjunto dos difeomorfismos simpléticos que possuem vizinhança na qual todos os difeomorfismos simpléticos possuem apenas pontos periódicos hiperbólicos, é exatamente o conjunto dos difeomorfismos simpléticos de Anosov. Mais ainda, ele mostra que genericamente no mundo simplético ou o difeomorfismo é Anosov, ou possui infinitos pontos periódicos 1-elípticos. 
Sendo assim, a ideia aqui é usar o caminho dado por Newhouse para criarmos tangência longe de difeomorfismos de Anosov e no momento certo usar o pasting lema para conseguirmos uma linearização na vizinhança do ponto periódico hiperbólico.

\section{Demonstração:}

Dado um ponto $p$ periódico hiperbólico para $f \in \mathcal{H}$, e $q \in H(p, g)$, definimos $u(q, g)$ como sendo o menor inteiro positivo $n$ tal que

$$
m\left(D f^{n}(q) \mid \tilde{E}_{q}^{u}\right) \geq 2,
$$

onde $\tilde{E}_{q}^{u}=T_{q} W^{u}(p, f)$ é a direção instável de $f$ no ponto $q$, e $m$ é a conorma da aplicação.

Para provarmos o Passo 1, precisamos do lema seguinte.

Lema B.0.4. Seja $f \in \mathcal{H}$, e p um ponto periódico hiperbólico de $f$. Se $f$ é um difeomorfismo não Anosov então existe uma sequência $q_{i} \in H(p, f)$ tal que

$$
\max \left\{u\left(q_{i}, f\right), u\left(q_{i}, f^{-1}\right)\right\} \rightarrow \infty, \quad \text { quando } i \rightarrow \infty
$$

Demonstração. Suponhamos por absurdo que não vale o lema. Sendo assim, existe um inteiro positivo $K>0$ tal que

$$
\sup _{q \in H(p, f)}\left\{u(q, f), u\left(q, f^{-1}\right)\right\} \leq K .
$$

Mostremos que com estas condições $f$ é Anosov, e assim temos uma contradição.

Primeiro vamos mostrar que o fecho dos pontos homoclínicos $\overline{H(p, f)}$ é um conjunto hiperbólico.

Dado $q \in H(p, f)$, consideremos $\tilde{E}_{q}^{s}=T_{q} W^{s}(p, f)$ e $\tilde{E}_{q}^{u}=T_{q} W^{u}(p, f)$. Onde $T_{q} W^{s(u)}(p, f)$ é o espaço tangente a curva $W^{s(u)}(p, f)$ no ponto $q$. Como $q$ é um ponto homoclínico transversal temos que $\tilde{E}_{q}^{s} \oplus \tilde{E}_{q}^{u}=T_{q} M$.

Direto da equação $(B .1)$ temos que para todo $q \in H(p, f)$

$$
m\left(D f^{K}(q) \mid \tilde{E}_{q}^{u}\right)>2 \text { e } m\left(D f^{-K}(q) \mid \tilde{E}_{q}^{s}\right)>2
$$

Afirmação 1: $\left.\| D f^{-K}(q) \mid \tilde{E}_{q}^{u}\right) \|<\frac{1}{2}$ e $\left.\| D f^{K}(q) \mid \tilde{E}_{q}^{s}\right) \|<\frac{1}{2}$.

De fato, pela equação (B.2), temos que

$$
\left|D f^{K}\left(f^{-K}(q)\right)\left(\frac{D f^{-K}(q)(v)}{\left|D f^{-K}(q)(v)\right|}\right)\right|>2, \text { para } v \in \tilde{E}_{q}^{u},
$$

de onde podemos concluir a Afirmação 1 na direção "instável". O outro caso é inteiramente análogo. 
Agora, seja $y \in \overline{H(p, f)}$. Podemos então escolher $q_{i} \in H(p, f)$ tais que $q_{i} \rightarrow y$, quando $i$ vai para infinito. Num senso de Grasmann, a menos de subsequência, podemos supor $\tilde{E}_{q_{i}}^{u} \rightarrow \tilde{E}_{y}^{u}$ e $\tilde{E}_{q_{i}}^{s} \rightarrow \tilde{E}_{y}^{s}$. Por propriedades de conorma e pela Afirmação 1, temos que $T_{y} M=\tilde{E}_{y}^{u} \oplus \tilde{E}_{y}^{s}$.

Afirmação 2: Se $q_{i}^{\prime} \in H(p, f)$ tal que $q_{i}^{\prime} \rightarrow y$ então $\tilde{E}_{q_{i}^{\prime}}^{u} \rightarrow \tilde{E}_{y}^{u}$ e $\tilde{E}_{q_{i}^{\prime}}^{s} \rightarrow \tilde{E}_{y}^{s}$.

De fato, suponhamos que exista um vetor unitário $v \notin \tilde{E}_{y}^{u}$ tal que $v$ seja ponto de convergência de vetores em $\tilde{E}_{q_{i}^{\prime}}^{u}$. Então $v=v_{1}+v_{2} \operatorname{com} v_{1} \in \tilde{E}_{y}^{u}$ e $v_{2} \in \tilde{E}_{y}^{s}$. Novamente por propriedades de conorma, temos que $\left|D f^{-K}(y)(v)\right| \geq 2$. E usando a Afirmação 1, temos que $\left|D f^{-K}(y)(v)\right| \leq \frac{1}{2}$, o que gera uma contradição provando que $\tilde{E}_{q_{i}^{\prime}}^{u} \rightarrow \tilde{E}_{y}^{u}$. Similarmente temos o outro caso, o que prova a Afirmação 2.

Mais ainda, temos que $m\left(D f^{K}(y) \mid \tilde{E}_{y}^{u}\right) \geq 2$ e $m\left(D f^{-K}(y) \mid \tilde{E}_{y}^{s}\right) \geq 2$

Pela Afirmação 2, a decomposição $T M=\tilde{E}^{u} \oplus \tilde{E}^{s}$ é contínua. Mostremos que de fato esta é uma decomposição hiperbólica para $\overline{H(p, f)}$.

Afirmação 3 Existe $C>0$ e $\lambda>1$ tal que $m\left(D f^{n}(y) \mid \tilde{E}_{y}^{u}\right) \geq C \lambda^{n}$ para todo $y \in \overline{H(p, f)}$.

Seja $K_{1}=\inf \left\{m\left(D f^{j}(y) \mid \tilde{E}_{y}^{u}\right) ; 0 \leq j \leq K, y \in \overline{H(p, f)}\right\}$. Da compacidade de $M$, continuidade da conorma, mais o fato de $f \in \operatorname{Diff}_{\omega}^{1}(M)$ temos que $K_{1}>0$.

Se $n<K$ temos que

$$
m\left(D f^{n}(y) \mid \tilde{E}_{y}^{u}\right) \geq \frac{K_{1}}{2} 2^{n / K} .
$$

Se $n \geq K$, seja $r \in \mathbb{N}$ e $0 \leq t<K$ tal que $n=r K+t$. Então,

$$
\begin{aligned}
m\left(D f^{n}(y) \mid \tilde{E}_{n}^{u}\right) & \geq 2^{r} m\left(D f^{t}(y) \mid \tilde{E}_{y}^{u}\right) \\
& \geq \frac{K_{1}}{2} 2^{n / K}
\end{aligned}
$$

Assim, tomando $C=\frac{K_{1}}{2}$ e $\lambda=2^{n / K}$ temos a Afirmação 3 .

Analogamente conseguimos uma estimativa para a direção $\tilde{E}^{s}$, e portanto temos que $\overline{H(p, f)}$ é um conjunto hiperbólico para $f, \operatorname{com} E^{s}=\tilde{E}^{s}$ e $E^{u}=\tilde{E}^{u}$.

Agora, mostrando que $\overline{H(p, f)}$ é aberto em $M$, como este é conexo, temos que $\overline{H(p, f)}=M$ e portanto $f$ é Anosov como queríamos. Para tal, usemos o fato de $\overline{H(p, f)}$ possuir estrutura de produto local. Dado $q \in H(p, f)$ como $f \in \mathcal{H}$

$$
W_{\delta}^{s(u)}(q, f) \subset W^{s(u)}(p, f) \subset \overline{H(p, f)},
$$

onde $\delta>0$ é dado pela propriedade de estrutura de produto local.

Agora, seja $y \in \overline{H(p, f)}$. Existem $q_{i} \in H(p, f)$ com $q_{i} \rightarrow y$, quando $i \rightarrow \infty$. Então, $W_{\delta}^{s(u)}\left(q_{i}\right) \rightarrow W_{\delta}^{s(u)}(y)$. E desde que $W_{\delta}^{s(u)}\left(q_{i}, f\right) \subset \overline{H(p, f)}$, temos

$$
W_{\delta}^{s(u)}(y, f) \subset \overline{H(p, f)} .
$$


E assim, como $\overline{H(p, f)}$ tem estrutura de produto local, conseguimos vizinhança $V \ni y$ em $M$ tal que $V \subset \overline{H(p, f)}$. Logo, $\overline{H(p, f)}$ é um aberto e fechado de $M$.

Demonstremos o passo 1.

Pelo Lema B.0.4 podemos tomar uma sequência $q_{i} \in H(p, f)$, tal que $u\left(q_{i}, f\right) \rightarrow \infty$. O caso $u\left(q_{i}, f^{-1}\right)$ é similar.

Como $\omega$ é uma 2-forma diferenciavel, $C^{\infty}$, temos que $\omega(p)$ é uma aplicação bilinear contínua. Logo $\omega(p)$ é limitada, isto é, existe $K_{p}$ tal que

$$
\left|\omega(p)\left(v_{1}, v_{2}\right)\right| \leq K_{p}\left|v_{1}\right|\left|v_{2}\right|, \text { para } p \in M \text {, e todo } v_{1}, v_{2} \in T_{p} M .
$$

E pela compacidade de $M$, temos que existe $K \in \mathbb{R}$ tal que

$$
\left|\omega(p)\left(v_{1}, v_{2}\right)\right| \leq K\left|v_{1}\right|\left|v_{2}\right|, p \in M, v_{1}, v_{2} \in T_{p} M
$$

Por escolha de $u\left(q_{i}, f\right)$, tomando $n_{i}=u\left(q_{i}, f\right)-1$ existe algum vetor unitário $v_{i} \in \tilde{E}^{u}\left(q_{i}, f\right)$ tal que $\left|D f^{n_{i}}\left(v_{i}\right)\right|<2$. Assim, dado $\varepsilon>0$ podemos usar o lema de Franks e encontrar $c>0$ tal que exista um difeomorfismo $f_{1 i} \varepsilon-C^{1}$ próximo de $f$ tal que

(a) $f(y)=f_{1 i}(y)$ para $y$ fora de uma pequena vizinhança de $\left\{q_{i}, f\left(q_{i}\right), \ldots, f^{n_{i}}\left(q_{i}\right)\right\}$;

(b) $f_{1 i}^{j}\left(q_{i}\right)=f^{j}\left(q_{i}\right)$ para $0 \leq j \leq n_{i}$;

(c) $q_{i} \in H\left(p, f_{1 i}\right)$;

(d) $\left|D f_{1 i}^{n_{i}}\left(q_{i}\right)\left(v_{i}\right)\right| \leq 2(1-c \varepsilon)^{n_{i}}$.

Afirmação 4: $\omega(q)\left|\tilde{E}_{q}^{u} \equiv 0 \equiv \omega(q)\right| \tilde{E}_{q}^{s}$, para todo $q \in H\left(p, f_{1 i}\right)$.

De fato, se $a_{1}, a_{2} \in \tilde{E}_{q}^{u}$ então $D f_{1 i}^{-n}(q)\left(a_{1}\right)$ e $D f_{1 i}^{-n}(q)\left(a_{2}\right)$ aproximam-se de zero quando $n$ cresce. No entanto, como $f_{1 i} \in \operatorname{Diff}_{\omega}^{1}(M)$

$$
\omega(q)\left(a_{1}, a_{2}\right)=\omega\left(f_{1 i}^{-n}(q)\right)\left(D f_{1 i}^{-n}\left(a_{1}\right), D f_{1 i}^{-n}(q)\left(a_{2}\right)\right), \text { para todo } n .
$$

Fazendo $n \rightarrow \infty$, temos que $\omega(q)\left(a_{1}, a_{2}\right)=0$. E portanto $\omega(q) \mid \tilde{E}_{q}^{u}=0$. Similarmente, temos que $\omega(q) \mid \tilde{E}_{q}^{s} \equiv 0$.

Desde que $\omega$ é não degenerada, existe vetor $v_{i}^{\prime} \in \tilde{E}^{s}\left(q_{i}, f_{1 i}\right)$ tal que $\omega\left(q_{i}\right)\left(v_{i}, v_{i}^{\prime}\right)=1$. Usando (B.3), temos

$$
\begin{aligned}
1=\omega\left(q_{i}\right)\left(v_{i}, v_{i}^{\prime}\right) & =\omega\left(f_{1 i}^{n_{i}}\left(q_{i}\right)\right)\left(D f_{1 i}^{n_{i}}\left(q_{i}\right)\left(v_{i}\right), D f_{1 i}^{n_{i}}\left(q_{i}\right)\left(v_{i}^{\prime}\right)\right) \\
& \leq K\left|D f_{1 i}^{n_{i}}\left(q_{i}\right)\left(v_{i}\right)\right|\left|D f_{1 i}^{n_{i}}\left(q_{i}\right)\left(v_{i}^{\prime}\right)\right| \\
& <2 K(1-c \varepsilon)^{n_{i}}\left|D f_{1 i}^{n_{i}}\left(q_{i}\right)\left(v_{i}^{\prime}\right)\right|
\end{aligned}
$$


daí,

$$
\left|D f_{1 i}^{n_{i}}\left(q_{i}\right)\left(v_{i}^{\prime}\right)\right|>(2 K)^{-1}(1-c \varepsilon)^{-n_{i}} .
$$

Usando mais uma vez o lema de Franks podemos perturbar $f_{1 i}$ para $f_{2 i} \in \operatorname{Diff}_{\omega}^{1}(M) \varepsilon-C^{1}$ próxima de $f_{1 i}$ tal que $f_{2 i}=f_{1 i}$ fora de uma vizinhança de $f_{1 i}^{-1}\left(q_{i}\right)$, exigindo que

(a) $f_{2 i}\left(f_{1 i}^{-1}\left(q_{i}\right)\right)=q_{i}$,

(b) $D f_{2 i}\left(f_{1 i}^{-1}\left(q_{i}\right)\right)\left(D f_{1 i}^{-1}\left(q_{i}\right)\left(v_{i}\right)\right)=\left(\alpha v_{i}+\beta v_{i}^{\prime}\right)$,

$\operatorname{com} \beta / \alpha>c \varepsilon$. Com isto, temos que $\left(\alpha v_{i}+\beta v_{i}^{\prime}\right) \in \tilde{E}^{u}\left(q_{i}, f_{2 i}\right)$, e $v_{i}^{\prime}$ continua pertencendo a $\tilde{E}^{s}\left(q_{i}, f_{2 i}\right)$.

Agora, $D f_{2}^{n_{i}}\left(q_{i}\right)\left(\alpha v_{i}+\beta v_{i}^{\prime}\right)$ pode ser escrito em coordenadas $\left(\xi_{1}, \xi_{2}\right)$, tal que

$$
\xi_{1} \in \tilde{E}^{u}\left(f_{1 i}^{n_{i}}\left(q_{i}\right), f_{1 i}\right) e \xi_{2} \in \tilde{E}^{s}\left(f_{1 i}^{n_{i}}\left(q_{i}\right), f_{1 i}\right) .
$$

Ou seja,

$$
\begin{aligned}
D f_{2 i}^{n_{i}}\left(q_{i}\right)\left(\alpha v_{i}+\beta v_{i}^{\prime}\right) & =D f_{1 i}^{n_{i}}\left(q_{i}\right)\left(\alpha v_{i}+\beta v_{i}^{\prime}\right) \\
& =\alpha D f_{1 i}^{n_{i}}\left(q_{i}\right)\left(v_{i}\right)+\beta D f_{1 i}^{n_{i}}\left(q_{i}\right)\left(v_{i}^{\prime}\right) .
\end{aligned}
$$

Assim, usando a condição (e) da primeira perturbação mais $(B .4)$, temos

$$
\frac{\left|\xi_{2}\right|}{\left|\xi_{1}\right|}=\frac{\left|\beta D f_{1 i}^{n_{i}}\left(q_{i}\right)\left(v_{i}^{\prime}\right)\right|}{\left|\alpha D f_{1 i}^{n_{i}}\left(q_{i}\right)\left(v_{i}\right)\right|}>\frac{\beta(2 K)^{-1}(1-c \varepsilon)^{-n_{i}}}{\alpha 2(1-c \varepsilon)^{n_{i}}}>c \varepsilon(4 K)^{-1}(1-c \varepsilon)^{-2 n_{i}} .
$$

Fazendo $i \rightarrow \infty$ temos que $n_{i} \rightarrow \infty$, e assim $\left|\xi_{1} / \xi_{2}\right| \rightarrow 0$. Daí, como $\tilde{E}^{s}\left(q_{i}, f_{i 2}\right)=\tilde{E}^{s}\left(q_{i}, f_{i 1}\right)$, temos que o ângulo entre $\tilde{E}^{u}\left(f_{i 2}^{n_{i}}\left(q_{i}\right), f_{i 2}\right)$ e $\tilde{E}^{s}\left(f_{i 2}^{n_{i}}\left(q_{i}\right), f_{i 2}\right)$ está indo para zero quando $i \rightarrow \infty$.

Logo $W^{s}\left(p, f_{i 2}\right)$ e $W^{u}\left(p, f_{i 2}\right)$ são $C^{1}$ próximos em $f_{i 2}^{n_{i}}\left(q_{i}\right)$, para $i$ grande o suficiente.

Agora, por continuidade das partes compactas das variedades estável e instável, e usando o pasting lema,podemos perturbar $f_{i 2}$ para $f_{i 3}$ tal que:

- $f_{i 3}=D f_{i 2}=D f$ numa vizinhança pequena da órbita de $p$, em coordenadas simpléticas local;

- $W^{s}\left(p, f_{i 3}\right)$ e $W^{u}\left(p, f_{i 3}\right)$ são $C^{1}$ próximos em $f_{i 3}^{n_{i}}\left(\tilde{q}_{i}\right)$, onde $\tilde{q}_{i} \in H\left(p, f_{i 3}\right)$ é um ponto homoclínico transversal perto de $q_{i}$.

Por fim, podemos usar uma última vez o lema de Franks e fazer uma $\varepsilon-C^{1}$ perturbação de $f_{i 3}$ para $f_{i 4}$, tal que $\tilde{q}_{i} \in H\left(p, f_{i 4}\right)$ e $W^{s}\left(\tilde{q}_{i}, f_{i 4}\right)$ e $W^{u}\left(\tilde{q}_{i}, f_{i 4}\right)$ exibam uma tangência em $f_{i 4}^{n_{i}}\left(\tilde{q}_{i}\right)$. E assim, podemos tomar $i$ suficientemente grande tal que $g_{1}=f_{i 4}$ seja $\varepsilon-C^{1}$ próximo de $f$. O que mostra o passo 1 . 



\section{Referências Bibliográficas}

[1] F. Abdenur, C. Bonatti e S. Crovisier, Nonunifom Hyperbolicity for $C^{1}$-generic diffeomorphisms, preprint (2008) arXiv:0809.3309. Em breve em "Israel Journal of Mathematics".

[2] F. Abdenur, C. Bonatti, S. Crovisier, L. Diaz e L. Wen, Periodic points and homoclinic classes, Ergod. Th. and Dynam. Sys. 27 (2007), 1-22.

[3] A. Arbieto e T. Catalan, Hyperbolicity in the Volume Preserving Scenario preprint (2010) arXiv:1004.1664.

[4] A. Arbieto e C. Matheus, A pasting lemma and some applications for conservative systems, Erg. Th. and Dynamic. Sys, 27 (2007), 1399-1417.

[5] M-C. Arnaud, Le "Closing Lemma"en topologie $C^{1}$, Supplément au Bull. Soc. Math. Fr., 74(1998).

[6] A. Ávila, On the regularization of conservative maps, preprint (2008) arXiv:0810.1533. Em breve em "Acta Matematica".

[7] M. Bessa, C. Ferreira e J. Rocha, On the stability of the set of hyperbolic closed orbits of a Hamiltonian, Preprint.

[8] M. Bessa e J. Rocha, Three-dimensional conservative star flows are Anosov, Discrete and Continuous Dynamical Systems, vol 26, 3, 839-846, 2010.

[9] C. Bonatti e S. Crovisier, Recurrence et generecite, Inv. Math. 158 (2004), 33-104

[10] C. Bonatti, L. Diaz e H. Pujals, A $C^{1}$-generic dichotomy for diffeomorphisms: Weak forms of hyperbolicity or infinitely many sinks or sources. Annals of Math. 158 (2003), pp. 355-418. 
[11] M Boyle e T. Downarowicz, The entropy theory of symbolic extensions, Inventiones Mathematicae, 156 (2004), 119-161.

[12] M Boyle, D. Fiebig e U. Fiebig, Residual entropy, conditional entropy, and subshift covers, Forum Math., 14 (2002), 713-757.

[13] R. Bowen, Equilibrium states and the ergodic theory of Anosov diffeomorphisms, Lecture Notes in Mathematic, No. 470, Springer-Verlag, N.Y. (1975).

[14] R. Bowen, Periodics Points and Measures for Axioma A Difeomorphisms, Tran. AMS , Vol. 54 (1971), 377-397.

[15] R. Bowen, Topological Entropy and Axiom A, Proc. Symp. Pure Math., AMS., Providence RI., 14 (1970), 23-41.

[16] D. Burguet, $C^{2}$ surface diffeomorphisms have symbolic extensions, preprint (2010) arXiv:0912.2018.

[17] J. Buzzi, Intrinsic ergodicity for smooth interval maps, Israel J. Math., 100 (1997), $125-161$.

[18] C. Carballo, C. Moralles e M. Pacifico, Homoclinic classes for generic $C^{1}$ vector fields, Ergod. Th. and Dynam. Sys. 23 (2003), pp. 403-415.

[19] T. Catalan e A. Tahzibi, A lower bound for topological entropy of generic non Anosov symplectic diffeomorphisms, Preprint (2010) arXiv:1011.2441.

[20] S. Crovisier, Perturbation de la dynamique de diffeomorphismes en topologie $C^{1}$. Preprint (2009).

[21] L. Diaz and T. Fisher, Symbolic extensions for partially hyperbolic diffeomorphisms, preprint (2009) arXiv:0906.2176, To appear in Discrete and Continuous Dynamical Systems.

[22] L. Diaz, T. Fisher, M. Pacífico, e J. Vieitez, Entropy-expansiveness for partially hyperbolic diffeomorphisms, preprint (2010) arXiv:1010.0721.

[23] T. Downarowicz e S. E. Newhouse, Symbolic extension and smooth dynamical systems, Inventiones Mathematicae, 160 (2005), 453-499.

[24] Franks, J. Necessary conditions for stability of diffeomorphisms. Trans. A.M.S. 158 (1971), 301-308.

[25] S. Gan, L. Wen, Nonsingular star flows satisfy Axiom A and the no-cycle condition, Invent. Math. 164 (2006), no. 2, 279-315.

[26] S. Gochenko, L. Shinikov, D. Turaev, On models with non-rough poincare homoclinic curves, Physica D 62(1993), 1-14. 
[27] S. Hayashi, Diffeomorphisms in $\mathcal{F}^{1}(M)$ satisfy Axiom A. Ergod. Th. and Dynamical Sys. 12(1992), 233-253.

[28] S. Hayashi, Connecting invariant manifolds and the solution of the $C^{1}$ stability and $\Omega$-stability conjectures for flows, Ann. of Math. (2) 145 (1997), no 1, 81-137.

[29] V. Horita e A. Tahzibi, Partial hyperbolicity for symplectic diffeomorphisms, Ann. I. H. Poincaré - AN 23 (2006), 641-661.

[30] V. Kaloshin, Generic diffeomorphisms with superexponential growth of number of periodic orbits, Commun. Math. Phys., 211, 253-271 (2000).

[31] A. Katok, Lyapunov exponents, entropy and periodic points for diffeomorphisms, Publications Mathématiques de l'IHES, 1980.

[32] C. Liang, G. Liu e W. Sun, Equivalent Conditions of Dominated Splittings for Volume-Preserving Diffeomorphism, Acta Math. Sinica 23 (2007), 1563-1576.

[33] S. Liao, A basic property of a certain class of differential systems (in Chinese). Acta Math. Sin. 22, 316-343(1979).

[34] G. Liao, J. Yang e M. Viana, Entropy of diffeomorphisms away from tangencies , Private communication.

[35] Mañé M. An Ergodic Closing Lemma. The Annals of Mathematics 2nd Ser., Vol 116, No. 3. (Nov., 1982), 503-540.

[36] Mañé M. A proof of the $C^{1}$ stability conjecture. Publ. Math. de IHES, Vol 66, (1987), 161-210.

[37] S. E. Newhouse, Topological entropy and Hausdorff dimension for area preserving diffeomorphisms of surfaces, Société Mathématique de France, Astérisque, 51 (1978), 323-334.

[38] S. E. Newhouse, Quasi-eliptic periodic points in conservative dynamical systems, American Journal of Mathematics, 99, No. 5 (1975), 1061-1087.

[39] S. E. Newhouse, Generic properties of conservative systems. In: G. Iooss, R.H.G. Helleman, R., Stora, eds., Chaotic behavior of deterministic systems, vol. XXXVI of Les Houches, pp. 443-451. North-Holland (1981)

[40] Palis, J. Global perspective for non-conservative dynamics. Annales I. H. Poincare Analyse Non Lineaire, v. 22. (2005), 485-507.

[41] Palis, J. On the $C^{1} \Omega$-stability conjecture. Inst. Hautes Études Sci. Publ. Math. No. 66 (1988), 211-215. 
[42] J. Palis e F. Takens, Hyperbolicity and sensitive-chaotic dynamics at homoclinic bifurcations, Cambridge: Cambridge University Press, 1993. (Cambridge Studies in Advanced Mathematics).

[43] M. Rees, A minimal positive entropy homeomorphism of the 2-torus, J. London Math. Soc. (2) 23 (1981), no. 3, 537-550

[44] R.C. Robinson, Generic properties of conservative systems, Amer. J. Math. 92 (1970), 562-603.

[45] K. Sigmund, Generic Properties of Invariant Measures for Axiom ADiffeomorphisms, Invent. Math. 11 (1970), 99-109.

[46] A. Tahzibi, $C^{1}$ Generic Pesin's entrpy formula, C. R. Acad. Sci. Paris, Ser. I 335 (2002), 1057-1062.

[47] Z. Xia, Homoclinic points in symplectic and Volume-Preserving diffeomorphisms, Communications in Mathematical Physics, 177 (1996), 435-449.

[48] Z. Xia e L. Wen, $C^{1}$ connecting lemmas, Trans. A.M.S., Vol. 352, No. 11 (2000) pp. 5213-5230.

[49] L. Young, Entropy of continuous flows on compact 2-manifolds, Topology, 16 (1977), no. $4,469-471$.

[50] E. Zehnder, Note on smoothing symplectic and volume-preserving diffeomorphisms, Geometry and topology (Proc. III Latin Amer. School of Math., Inst. Mat. Pura Aplicada CNPq, Rio de Janeiro, 1976), pp. 828-854. Lecture Notes in Math., Vol. 597, Springer, Berlin, 1977. 


\section{Tabela de Símbolos e Abreviações}

$M \quad$ Variedade Riemanianna, compacta, conexa, de dimensão $d$ ou $2 n$

$\omega \quad 2$-forma diferenciável, $C^{\infty}$, fechada, e não degenerada, sobre $M$

$m \quad$ forma de volume sobre $M$

$\operatorname{Diff}^{r}(M)$ conjunto dos difeomorfismos de classe $C^{r}$ sobre $M, r \geq 1$, munido da topologia $C^{r}$

$\operatorname{Diff}_{\omega}^{1}(M)$ conjunto dos difeomorfismos de classe $C^{1}$, que preservam a 2-forma $\omega$

$\operatorname{Diff}_{m}^{1}(M)$ conjunto dos difeomorfismos de classe $C^{1}$, que preservam volume sobre $M$

$h(f) \quad$ entropia topológica de $f$

$\mathcal{M}(f) \quad$ conjunto das medidas de probabilidade de $f$

$h_{\mu}(f) \quad$ entropia métrica de $f$ com respeito a $\mu \in \mathcal{M}(f)$

$\operatorname{Per}(f) \quad$ conjunto dos pontos periódicos de $f$

$\tau(p, f) \quad$ período do ponto periódico $p$ de $f$

$H_{n}(f) \quad$ conjunto dos pontos periódicos hiperbólicos de $f$, com período menor ou igual a $n$

$H(f) \quad$ conjunto dos pontos periódicos hiperbólicos de $f$

$W^{u}(p, f) \quad$ variedade instável de $p$ para $f$

$W^{s}(p, f) \quad$ variedade estável de $p$ para $f$

$\Lambda_{i}(p, f) \quad$ conjunto dos pontos periódicos hiperbólicos com índice $i$ 
$H(p, f)$ conjunto dos pontos homoclínicos transversais de $p$, com respeito a $f$

$\Lambda(g) \quad$ continuação do conjunto hiperbólico $\Lambda$ para $g$

$p(g) \quad$ continuação do ponto periódico hiperbólico $p$ para $g$

$\lambda(p, f) \quad$ valor absoluto do menor autovalor dentre os autovalores com valor absoluto maior do que um da aplicação $D f_{p}^{\tau(p, f)}$, para $p \in H(f)$

$\chi(p, f) \quad$ menor expoente de lyapunov positivo de $p \in H(f)$

$s_{n}(f) \quad \sup \chi(p, f)$ sobre todos os pontos $p \in H_{n}(f)$

$s(f) \quad \sup \chi(p, f)$ sobre todos os pontos periódicos hiperbólicos de $f$

$B_{\varepsilon}(x) \quad$ bola de centro $x$ e raio $\varepsilon$

$\Omega(f) \quad$ conjunto dos pontos não errantes de $f$.

$\mathcal{H} \quad$ conjunto residual dado por Xia

$\tau(f) \quad$ menor período dentre os períodos dos pontos periódicos hiperbólicos de $f$

$\chi(f) \quad \sup \chi(p, f)$ sobre os pontos periódicos hiperbólicos com período $\tau(f)$

$C(E, x)$ componente conexa de $E$ contendo $x$

$\sigma($.$) \quad aplicação shift$

$P_{f}(\phi) \quad$ pressão topológica de $f$, com respeito ao observável $\phi$

$S_{f} \quad$ função geradora do difeomorfismo simplético $f$ 



\section{Índice Remissivo}

índice de um ponto periódico hiperbólico, 33

assintoticamente h-expansivo, 4

campo vetorial Hamiltoniano, 32

ciclo heterodimensional, 34

Closing Lema, 11

conjunto hiperbólico, 2

connecting Lema, 10

conorma, 52

coordenadas simpléticas, 9

decomposição dominada, 36

difeomorfismo

de Anosov, 2

simplético, 3

entropia

topológica, 1

entropia residual, 20

Ergodic closing Lema, 11

extensão

simbólica , 4

simbólica principal, 4

função geradora, 8

homoclinicamente relacionado, 35

Lema de Franks, 7 medida

hiperbólica, 16

periódica, 14

ortogonal simplético, 23

partição essencial, 20

pasting lema, 9

ponto

homoclínico transversal, 17

não errante, 11

periódico hiperbólico, 2

sistema linear periódico, 35

subconjunto residual, 3

subespaço lagrangiano, 23

subvariedade Lagrangiana, 23

tangência homoclínica, 17

Teorema de Newhouse, 3

variedade estável, 10

vetor de Lyapunov, 17 